\title{
Divergent Adsorption Behavior Controlled by Primary Coordination Sphere Anions in the Metal-Organic Framework $\mathrm{Ni}_{2} \mathrm{X}_{2}$ BTDD
}

Julius J. Oppenheim ${ }^{\mathrm{a}}$, Jenna L. Mancuso ${ }^{\mathrm{b}}$, Ashley M. Wrighta, Adam J. Rieth ${ }^{\mathrm{a}}$, Christopher H. Hendon $^{\text {b*}}$, Mircea Dincă ${ }^{a *}$

aDepartment of Chemistry, Massachusetts Institute of Technology, 77 Mass. Ave. Cambridge, Massachusetts, 02139, United States

${ }^{b}$ Materials Science Institute, Department of Chemistry and Biochemistry, University of Oregon, Eugene, Oregon 97403, United States

$\ddagger$ These authors contributed equally.

\section{Supplementary Information}

\section{Table of Contents}

1. General information 1

2. Computations 3

3. Diffuse reflectance infrared Fourier transform (DRIFTS)

4. Non-classical metal carbonyl $\Delta \mathrm{H}-v$ Correlation 8

5. Typical isosteric enthalpies of adsorption for $\mathrm{CO}, \mathrm{C}_{2} \mathrm{H}_{4}$

6. Isosteric enthalpy of adsorption calculations $\quad 11$

6.1. Description of Models 11

6.2. Unilan model fits 14

6.3. Virial model fits $\quad 21$

6.4. Sips model fits 28

6.5. DSL model fits 35

6. Mathematica notebook for calculation of isosteric enthalpy of adsorption 42

7. References

\section{Section S1. General Information.}

Materials and Methods. $\mathrm{NiCl}_{2} \cdot 6 \mathrm{H}_{2} \mathrm{O}$ (Strem Chemicals), $\mathrm{HCl}$ (32-35\%, $\mathrm{BDH}$ - VWR Analytic) methanol (99.9\%, VWR), N,N-dimethylformamide (99.8\%, Millipore), 1-chloro-2,4-dinitrobenzene (99\%, TCI), catechol (99\%, Sigma-Aldrich), $\mathrm{K}_{2} \mathrm{CO}_{3}$ (99\%, Sigma-Aldrich), Sn Powder (150 micron, 99.5\%, SigmaAldrich), diethyl ether (99\%, Sigma-Aldrich), trimethylsilyl bromide (97\%, SigmaAldrich), acetone (ACS grade, Macron Chemical), fuming $\mathrm{HNO}_{3}\left(90 \%\right.$ min, Macron), $\mathrm{H}_{2} \mathrm{SO}_{4}$ (95-98\%, BDH Chemicals), acetic acid (ACS grade, VWR BDH Chemicals), $\mathrm{NaNO}_{2}$ (98\%, Alfa Aesar), ethanol (200 proof, Koptec), $\mathrm{KOH}$ (ACS grade, BDH chemicals), CsF (99\%, Beantown Chemicals) were used as received. Benzene (ACS grade, EMD) was purged with argon, and subjected to three freeze-pump-thaw cycles before bringing into a nitrogen-filled glovebox and stored over $3 \AA$ molecular sieves. The MOFs used in this study (1$\mathbf{F}, 1-\mathrm{Cl}, 1-\mathrm{Br}, 1-\mathrm{OH}$ ) were from the same batch as those reported by Rieth et al. and further characterization can be found therein. ${ }^{1}$

Gas adsorption isotherms were measured by a volumetric method using a Micromeritics ASAP 2020 gas sorption analyzer. Typical samples of ca. $40-80 \mathrm{mg}$, preactivated at $>100^{\circ} \mathrm{C}$ to remove all residual 
solvent, were transferred in an Ar-filled glovebox to a pre-weighed analysis tube. The tube with sample inside was weighed again to determine the mass of the sample. The tube was capped with a Micromeritics TranSeal ${ }^{\mathrm{TM}}$, brought out of the glovebox, and transferred to the analysis port of the gas sorption analyzer. Free space correction measurements were performed using ultra-high purity He gas (UHP grade 5, 99.999\% pure). Nitrogen isotherms were measured using UHP grade Nitrogen. All nitrogen analyses were performed using a liquid nitrogen bath at $77 \mathrm{~K}$. Carbon monoxide and ethylene isotherms were measured at varying temperatures in a water/ethylene glycol isothermal bath using high purity gases (carbon monoxide, 99.99\%; ethylene 99.9\%). Hydrogen $\left(\mathrm{H}_{2}, 99.999 \%\right)$ isotherms were performed using a liquid nitrogen bath $(77 \mathrm{~K})$ or a liquid argon bath $(87 \mathrm{~K})$. Oil-free vacuum pumps were used to prevent contamination of sample or feed gases.

Diffuse reflectance infrared Fourier transform spectroscopy (DRIFTS) measurements were performed using a Bruker Tensor 37 IR spectrometer equipped with a liquid nitrogen cooled mercury cadmium telluride detector and a Pike DiffusIR accessory. A sample of MOF, pre-activated under vacuum to remove all solvent, was diluted with $\mathrm{KBr}$ in a ratio of approximately 1:50-100 (MOF:KBr) in an argon-filled glovebox. The resulting solid solution was then packed into a ceramic cup and sealed in the DiffusIR cell. The cell was brought out of the box, and a static dry spectrum was recorded with the cell sealed. Known volumes of $\mathrm{CO}$ was dosed into the cell through an airtight port using a gas tight syringe. The temperature for all measurements was $20^{\circ} \mathrm{C}$. Data was transformed using the KubelkaMunk function.

Computational Methods. All structures were fully optimized within the DFT construct as implemented in the Vienna Ab-Initio Software Package (VASP) ${ }^{2}$ using the PBEsol ${ }^{3}$ GGA functional. A projectaugmented-wave plane wave basis set was employed with a $500 \mathrm{eV}$ plane-wave cutoff. To prevent anomalous phasing interactions within adsorbate binding models a $1 \times 1 \times 1 \mathrm{k}$-grid was used. Spinpolarized calculations were performed and a high-spin state was enforced on $\mathrm{Ni}$ atoms consistent with our previous study. ${ }^{1}$ The energy of "free" adsorbates were computed using the same methods in a unit cell containing $>20 \AA$ of vacuum. Binding energies were computed from the difference in energy between empty frameworks plus the adsorbate and the adsorbate-bound frameworks. The atomic charges were obtained using a Mulliken-like charge analysis performed by default in VASP. The partitioning Wigner-Seitz radii was kept at the default setting (controlled by the flag RWIGS). As a result, an increase in charge density on, for example, $\mathrm{Ni}^{2+}$, indicates that the inner sphere ligands are forming shorter, higher electron density, bonds with the Ni centers. 


\section{Section S2. Computations.}

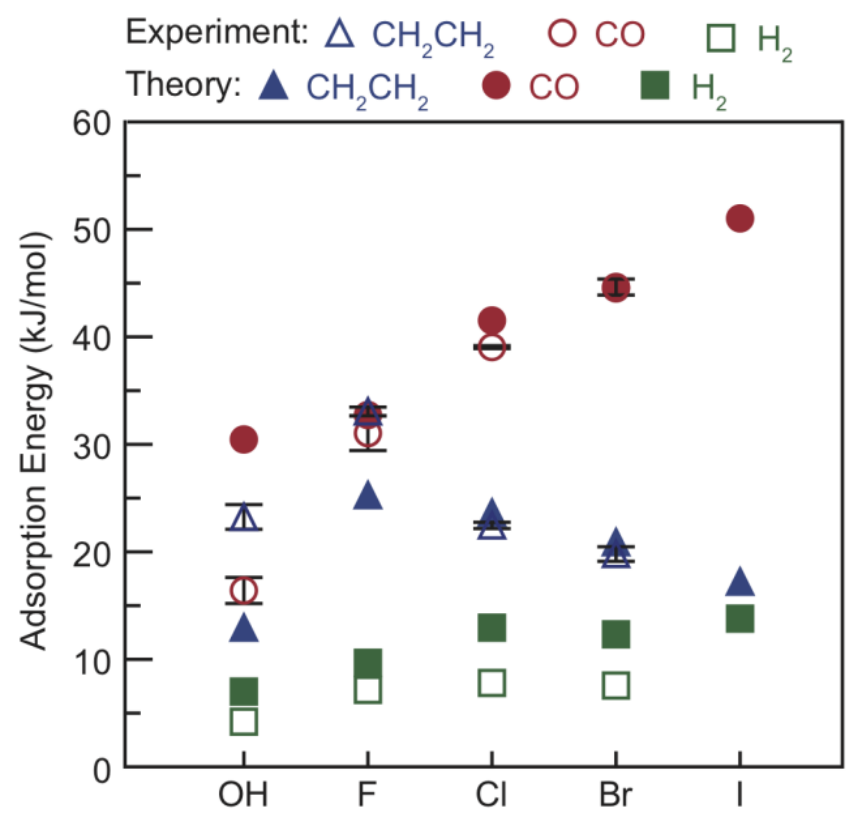

Figure S2.1: Comparison between the experimentally determined isosteric adsorption enthalpies for carbon monoxide (blue empty triangles), ethylene (red empty circles), and dihydrogen (green empty squares) with the theoretical adsorption energies computed at the HSEsol06 level of theory from PBED3 optimized geometries (solid shapes) show excellent agreement for halide derivatives, but some disparity in the case of hydroxyl ligands and adsorbates with pi-systems. 
Table S2.1: Comparative tabulation of adsorption energies recovered from experiment and theory for halide exchanged frameworks.

\begin{tabular}{|c|c|c|c|}
\hline & Adsorbate & Experiment & Theory (HSE06) \\
\hline \multirow{3}{*}{$\mathrm{OH}$} & $\mathrm{CO}$ & $16.42 \pm 1.17$ & 31.79 \\
\hline & $\mathrm{H}_{2}$ & $7.00 \pm 0.01$ & 4.19 \\
\hline & $\mathrm{C}_{2} \mathrm{H}_{4}$ & $23.23 \pm 1.14$ & 13.06 \\
\hline \multirow{3}{*}{$\mathbf{F}$} & $\mathrm{CO}$ & $31.00 \pm 1.61$ & 32.74 \\
\hline & $\mathrm{H}_{2}$ & $7.09 \pm 0.02$ & 9.78 \\
\hline & $\mathrm{C}_{2} \mathrm{H}_{4}$ & $32.98 \pm 0.40$ & 25.07 \\
\hline \multirow{3}{*}{$\mathrm{Cl}$} & $\mathrm{CO}$ & $39.02 \pm 0.16$ & 41.44 \\
\hline & $\mathrm{H}_{2}$ & $7.63 \pm 0.01$ & 12.90 \\
\hline & $\mathrm{C}_{2} \mathrm{H}_{4}$ & $22.31 \pm 0.24$ & 23.62 \\
\hline \multirow{3}{*}{$\mathrm{Br}$} & $\mathrm{CO}$ & $44.56 \pm 0.75$ & 44.42 \\
\hline & $\mathrm{H}_{2}$ & $7.62 \pm 0.01$ & 12.38 \\
\hline & $\mathrm{C}_{2} \mathrm{H}_{4}$ & $19.8 \pm 0.65$ & 20.79 \\
\hline \multirow{3}{*}{ I } & $\mathrm{CO}$ & - & 51.00 \\
\hline & $\mathrm{H}_{2}$ & - & 13.83 \\
\hline & $\mathrm{C}_{2} \mathrm{H}_{4}$ & - & 17.14 \\
\hline
\end{tabular}

Table S2.2: Total valence electrons on nickel atom for the empty framework and those with carbon monoxide and ethylene bound showing an increase in electron density with $\sigma$-donation from $\mathrm{CO}$ and a decrease in electron density associated with $\pi$-backbonding from ethylene.

\begin{tabular}{|c|c|c|c|}
\hline & Model & Electrons on $\mathrm{Ni}$ & Spin on $\mathrm{Ni}$ \\
\hline \multirow{3}{*}{$\mathrm{OH}$} & $\mathrm{CO}$ & 9.268 & 1.573 \\
\hline & Empty & 9.148 & 1.630 \\
\hline & $\mathrm{C}_{2} \mathrm{H}_{4}$ & 9.160 & 1.631 \\
\hline \multirow{4}{*}{$\mathbf{F}$} & $\mathrm{CO}$ & 9.211 & 1.628 \\
\hline & $\mathrm{H}_{2}$ & 9.117 & 1.662 \\
\hline & Empty & 9.126 & 1.665 \\
\hline & $\mathrm{C}_{2} \mathrm{H}_{4}$ & 9.012 & 1.663 \\
\hline \multirow{4}{*}{$\mathrm{Cl}$} & $\mathrm{CO}$ & 9.241 & 1.556 \\
\hline & $\mathrm{H}_{2}$ & 9.135 & 1.598 \\
\hline & Empty & 9.128 & 1.606 \\
\hline & $\mathrm{C}_{2} \mathrm{H}_{4}$ & 9.031 & 1.607 \\
\hline \multirow{4}{*}{$\mathrm{Br}$} & $\mathrm{CO}$ & 9.238 & 1.526 \\
\hline & $\mathrm{H}_{2}$ & 9.121 & 1.547 \\
\hline & Empty & 9.102 & 1.588 \\
\hline & $\mathrm{C}_{2} \mathrm{H}_{4}$ & 9.004 & 1.595 \\
\hline \multirow{4}{*}{ I } & $\mathrm{CO}$ & 9.247 & 1.470 \\
\hline & $\mathrm{H}_{2}$ & 9.114 & 1.526 \\
\hline & Empty & 9.081 & 1.553 \\
\hline & $\mathrm{C}_{2} \mathrm{H}_{4}$ & 8.972 & 1.562 \\
\hline
\end{tabular}




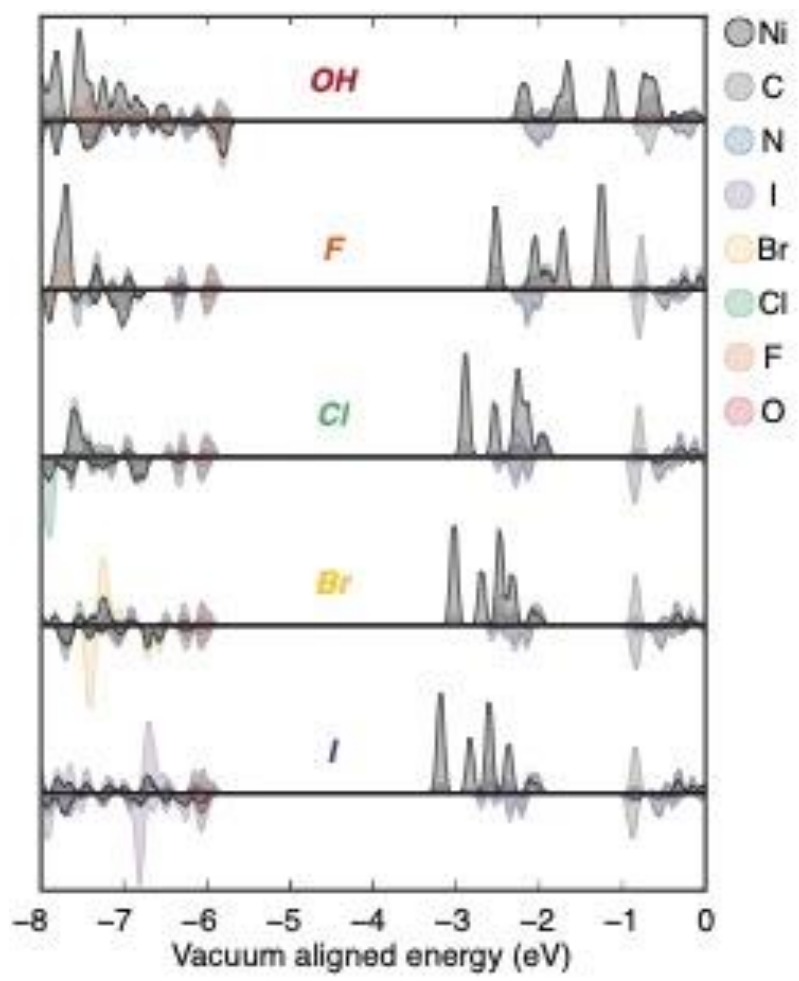

Figure S2.2. Vacuum aligned atom-projected density of states (DOS) plots at the HSEsol06 level of theory for each $\mathrm{Ni}_{2} \mathrm{X}_{2} \mathrm{BTDD}$ species theoretically investigated reveal that the reduction in ligand field strength down the group of halides modifies the conduction band potential so the band gap is reduced. The reduced potential of unoccupied $d$-states is a barometer for adsorption trends, however the major consideration for $\pi$-acid adsorption strength turns out to be whether the molecule functions as a $\sigma$-donor or $\pi$-acceptor. 

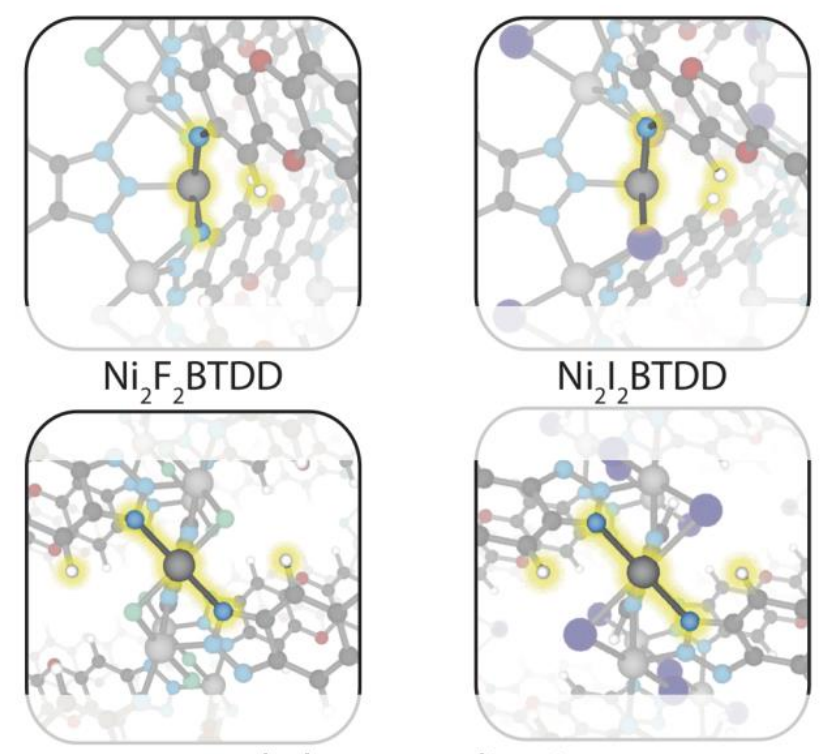

ethylene coordination
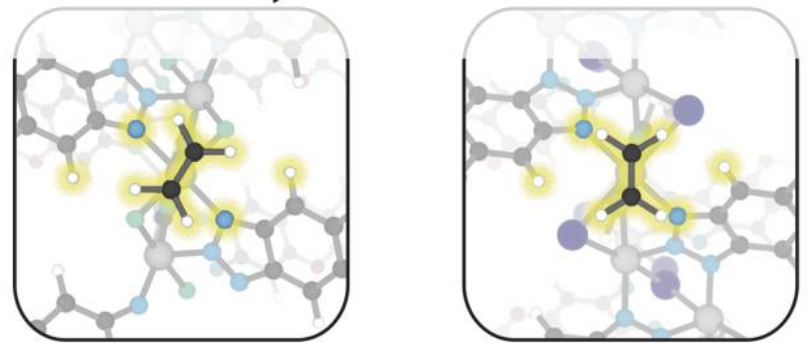

Figure S2.3: Definition of linker-linker dihedral angle. The smaller this dihedral angle, the less "open" the binding pocket is due to steric hinderance from the BTDD.

Table S2.3: Structure parameters for active site "openness" as a function of halide exchange recovered from structures fully equilibrated with the PBE-D3 functional.

\begin{tabular}{|c|c|c|}
\hline & Ni-Ni distance & Linker-Linker dihedral \\
\hline OH & $3.11 \AA$ & $113.0^{\circ}$ \\
\hline $\mathbf{F}$ & $3.10 \AA$ & $109.8^{\circ}$ \\
\hline $\mathrm{Cl}$ & $3.24 \AA$ & $102.6^{\circ}$ \\
\hline $\mathrm{Br}$ & $3.29 \AA$ & $97.3^{\circ}$ \\
\hline $\mathbf{I}$ & $3.37 \AA$ & $88.3^{\circ}$ \\
\hline
\end{tabular}


Section S3. Diffuse reflectance infrared Fourier transform (DRIFTS)

To a sample of 1-Cl (2.6 mg, $0.0057 \mathrm{mmol}), 750 \mu \mathrm{L}$ of $\mathrm{CO}(\sim 3$ eq.) were dosed in and a DRIFT spectrum was recorded 5 times in increments of 120 seconds

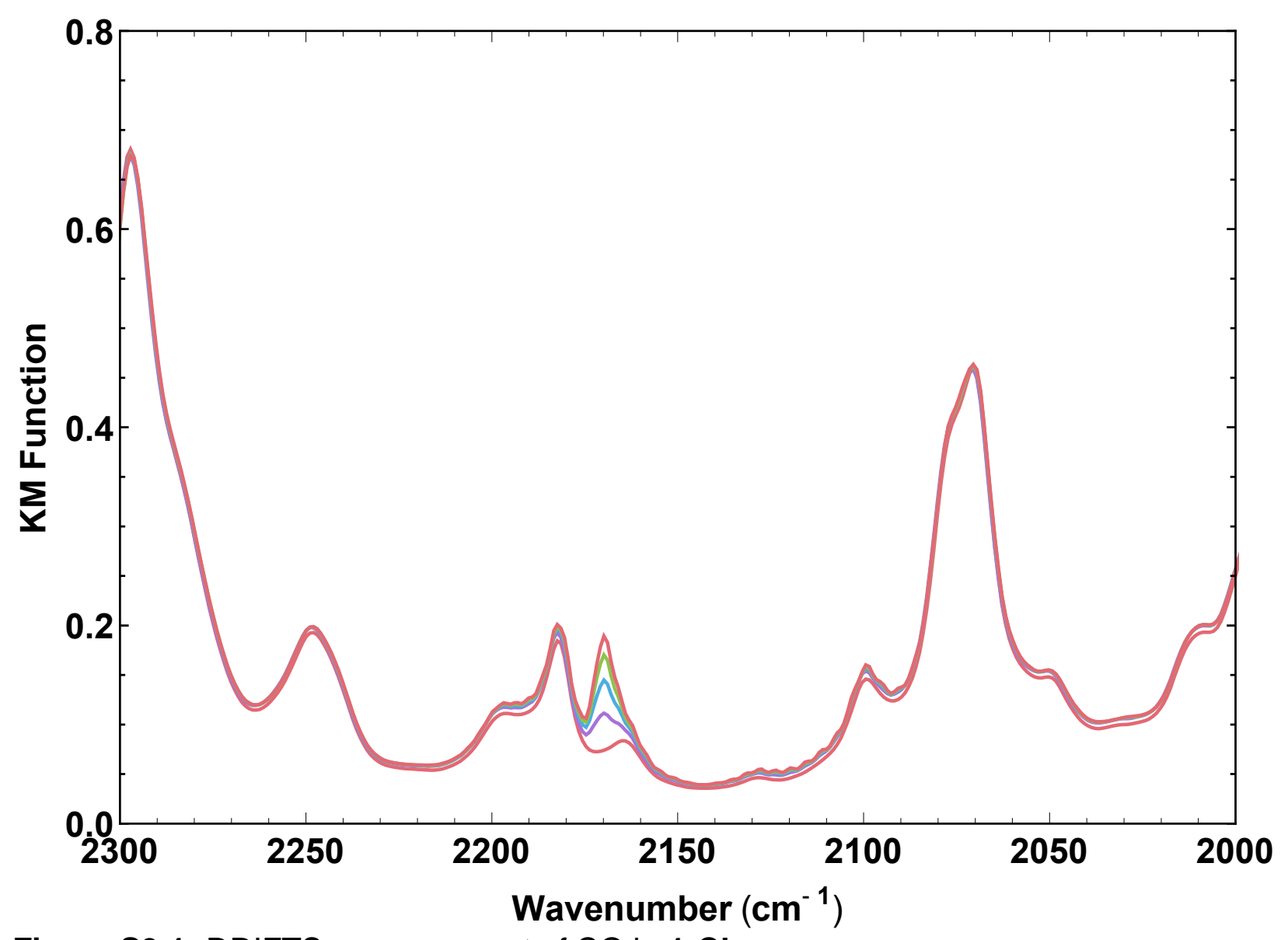

Figure S3.1: DRIFTS measurement of $\mathrm{CO}$ in 1-CI. 


\section{Section S4. Non-classical Metal Carbonyl Correlation}

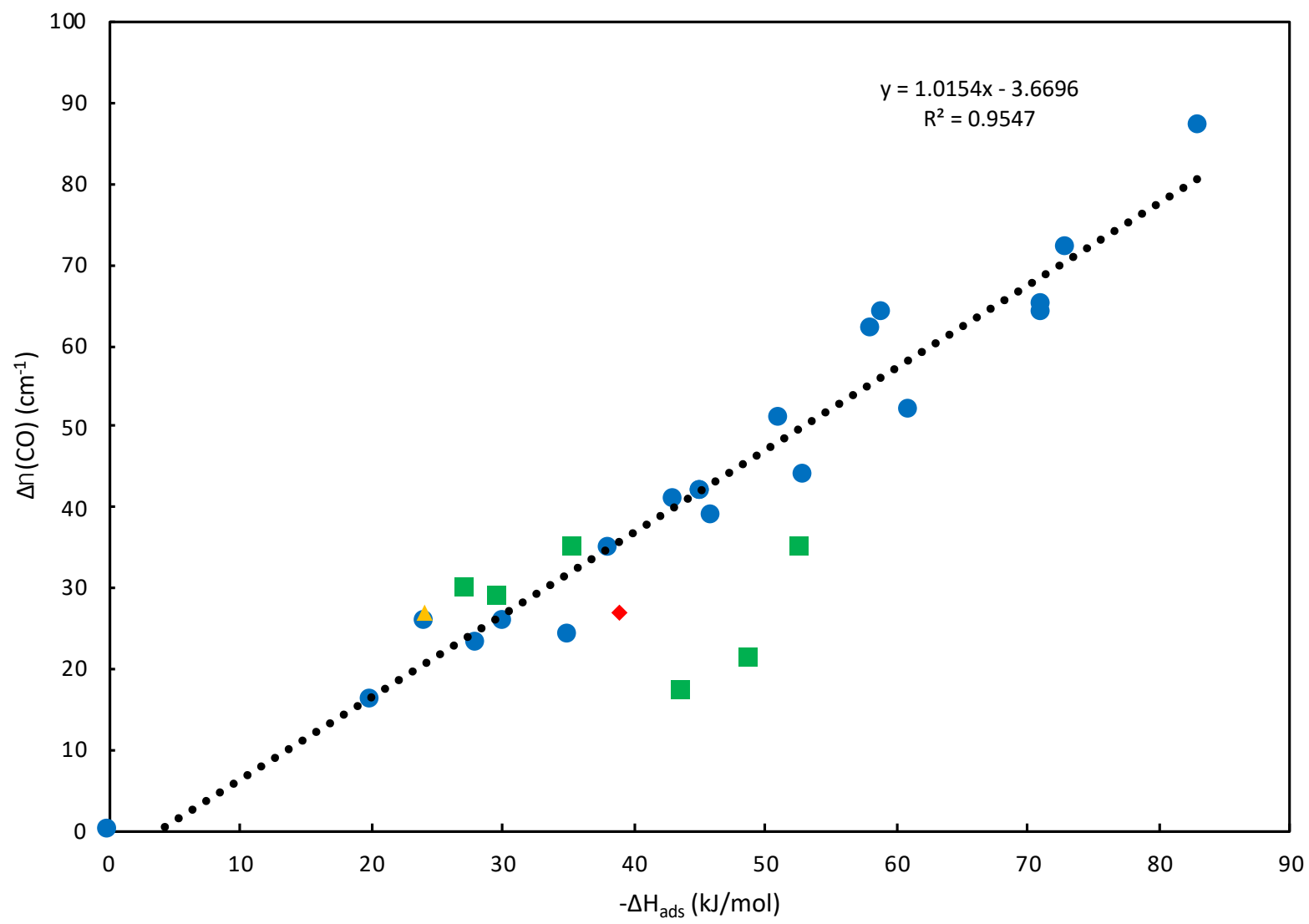

Figure S4.1: $\Delta v-\Delta \mathrm{H}_{\text {ads }}$ linear relationship: red diamond (1-Cl, this work), blue circle ${ }^{4}$, green square ${ }^{5}$, yellow triangle ${ }^{6}$. 
Section S5. Typical isosteric enthalpies

Table S5.1: Selection of literature isosteric enthalpy of adsorption values for $\mathrm{CO}$.

\begin{tabular}{|c|c|c|}
\hline Framework & $\begin{array}{l}\mathrm{CO}-\Delta \mathrm{H}_{\mathrm{ads}} \\
(\mathrm{kJ} / \mathrm{mol})\end{array}$ & Method/Notes ${ }^{a}$ \\
\hline Zeolite $5 A^{7}$ & 70 & One of Langmuir or Freundlich, $194.5 / 237 / 298 \mathrm{~K}$ \\
\hline Zeolite $13 X^{7}$ & 70 & One of Langmuir or Freundlich, 194.5/237/298 K \\
\hline $\mathrm{Ni}-\mathrm{MOF}-74^{5}$ & 52.7 & $\begin{array}{l}\text { Two or three site(?) temperature dependent } \\
\text { Langmuir model, } 298 / 308 / 318 \mathrm{~K}\end{array}$ \\
\hline $\mathrm{Cu}(\mathrm{I}) / \mathrm{MIL}-100(\mathrm{Fe})^{8}$ & 50 & DSLF, 288/298/308 K \\
\hline $\mathrm{Cu}$ doped $\mathrm{Al}_{2} \mathrm{O}_{3}{ }^{9}$ & 48.8 & Modeling kinetics with linear driving force model \\
\hline Co-MOF- $74^{5}$ & 48.8 & $\begin{array}{l}\text { Two or three site(?) temperature dependent } \\
\text { Langmuir model, } 298 / 308 / 318 \mathrm{~K}\end{array}$ \\
\hline Fe-MOF-74 & 43.6 & $\begin{array}{l}\text { Two or three site(?) temperature dependent } \\
\text { Langmuir model, } 298 / 308 / 318 \mathrm{~K}\end{array}$ \\
\hline MIL-100(Fe) ${ }^{8}$ & 38 & DSLF, 288/298/308 K \\
\hline Mg-MOF-74 5 & 35.4 & $\begin{array}{l}\text { Two or three site(?) temperature dependent } \\
\text { Langmuir model, } 298 / 308 / 318 \mathrm{~K}\end{array}$ \\
\hline Mn-MOF- $74^{5}$ & 29.7 & $\begin{array}{l}\text { Two or three site(?) temperature dependent } \\
\text { Langmuir model, } 298 / 308 / 318 \mathrm{~K}\end{array}$ \\
\hline Mg-MOF-74 ${ }^{10}$ & 29 & Variable temperature IR, $-\Delta \mathrm{S}^{0}=133 \mathrm{~J} / \mathrm{molK}$ \\
\hline Zn-MOF-745 & 27.2 & $\begin{array}{l}\text { Two or three site(?) temperature dependent } \\
\text { Langmuir model, } 298 / 308 / 318 \mathrm{~K}\end{array}$ \\
\hline Zn-MOF-177 7 & 22 & One of Langmuir or Freundlich, $194.5 / 237 / 298 \mathrm{~K}$ \\
\hline Zn-MOF-57 & 16 & One of Langmuir or Freundlich, 194.5/237/298 K \\
\hline $\mathrm{Ag}$ doped $\mathrm{Al}_{2} \mathrm{O}_{3}{ }^{9}$ & $37.3 / 36.1$ & Modeling kinetics with linear driving force model \\
\hline
\end{tabular}

${ }^{\text {a }}$ DSL $=$ Dual-site Langmuir, DSLF $=$ Dual-site Langmuir-Freundlich $=$ Dual-site Sips

Table S5.2: Selection of literature isosteric enthalpy of adsorption values for ethylene.

\begin{tabular}{|c|c|c|}
\hline Framework & $\begin{array}{l}\mathrm{C}_{2} \mathrm{H}_{4}-\Delta \mathrm{H}_{\text {ads }} \\
(\mathrm{kJ} / \mathrm{mol})\end{array}$ & Method/Notes \\
\hline $\begin{array}{l}\text { Cr-MIL-101- } \\
\mathrm{SO}_{3} \mathrm{Ag}^{11}\end{array}$ & 120 & DSLF, 293/303/313 K \\
\hline PAF-1-SO ${ }_{3} \mathrm{Ag}^{12}$ & 106 & DSLF, $296 / 318 \mathrm{~K}, \mathrm{Ag}$ on PAF \\
\hline $\begin{array}{l}\text { MIL-101-Cr- } \\
\mathrm{SO}_{3} \mathrm{Ag}^{13}\end{array}$ & 63 & DSLF, 296/318 K \\
\hline HUS-6(Hf)-Ag ${ }^{14}$ & 56.5 & Virial, 283/298 K \\
\hline Co-MOF-74 ${ }^{15}$ & 47 & Virial, $298 / 308 / 318 \mathrm{~K}$ \\
\hline Fe-MOF-74 $4^{16}$ & 45 & $\begin{array}{l}\text { DSLF, 318/333/353 K, Caption for Fig. S3 is } \\
\text { confusing }\end{array}$ \\
\hline Mg-MOF-74 ${ }^{17}$ & 42.6 & DSLF, 278/298/318 K \\
\hline
\end{tabular}




\begin{tabular}{|l|r|l|}
\hline $\mathrm{Cu}^{+} @ \mathrm{Cr}-\mathrm{MIL}-101^{18}$ & 40 & DSL, 303/313/323 K, CuCl loaded \\
\hline $\mathrm{Cr}-\mathrm{MIL}-101-\mathrm{SO}_{3} \mathrm{H}^{11}$ & 35 & DSLF, 298/303/313 K \\
\hline $\mathrm{Cu}-\mathrm{MOF}-74^{15}$ & 30 & Virial, 298/308/318 K \\
\hline Fe-MIL-142A & 26 & $\begin{array}{l}\text { DSLF, 278/288/298 K, typo in text body, value taken } \\
\text { from figure }\end{array}$ \\
\hline PAF-1-SO ${ }_{3} \mathrm{H}^{12}$ & 23 & DSLF, 296/318 K, PAF \\
\hline Fe-PCN-250 & 21.1 & DSLF, 273/298 K \\
\hline PAF-1 & 14 & DSLF, 296/318 K, PAF, also known as PPN-6 \\
\hline Cr-MIL-101-SO $_{3} \mathrm{H}^{13}$ & 10 & DSLF, 296/318 K \\
\hline
\end{tabular}




\section{Section S6. Isosteric enthalpy of adsorption calculations}

\section{Section 6.1. Description of Models}

The Langmuir model is defined as $n=\frac{U_{1} a_{1} p}{1+a_{1} p}$, where $n$ is the quantity of adsorbate adsorbed onto the adsorbent surface and $p$ is the pressure. This model can be derived from the steady state of $\frac{d n}{d t}=k_{1} p\left(U_{1}-n\right)-k_{2} n$, in which the rate of adsorption is limited by the number of available sites. ${ }^{21}$ The Langmuir model can be extended to a heterogeneous system in which there are two adsorption sites to form the Dual-Site Langmuir model, $n=\frac{U_{1} a_{1} p}{1+a_{1} p}+\frac{U_{2} a_{2} p}{1+a_{2} p}$. Chemically a two site model in the $\mathrm{Ni}_{2} \mathrm{X}_{2} \mathrm{BTDD}$ system can be interpreted as distinct adsorption to the Ni sites and the BTDD sites, however this interpretation is not necessarily precise. Distinct adsorption sites may be added to the model until the limit of continuous energy distribution is reached. In this limit, the Unilan isotherm is derived, $n=\frac{\int_{-s}^{+s} \frac{U e^{j} p}{a+e} d j}{\int_{-s}^{+s} d j}=\frac{U}{2 s} \ln \frac{1+p e^{s} / a}{1+p e^{-s} / a}$. The Sips (also known as Langmuir-Freundlich) isotherm is a mixture of the Langmuir and Freundlich isotherms, $n=\frac{U a p^{s}}{1+a p^{s}}$. In this model, the factor $s$ introduces heterogeneity. ${ }^{22}$ These particular models were chosen as the inverse, $P(n)$, can be expressed analytically, which allows for facile error propagation. The errors of the isotherm fits were calculated using the variance-covariance matrix of the fit, $\Sigma^{f}=J \Sigma^{X} J^{T}$, where $\Sigma^{f}$ is a vector of the variance for each variable, $\mathrm{J}$ is the Jacobian $\left(\frac{d F}{d a_{i}}\right)$, and $\Sigma^{X}$ is the variance-covariance matrix. ${ }^{23}$ For each of these models, the isosteric enthalpy of adsorption is calculated using the Clausius-Clapeyron relation, $\ln P(n)=\frac{\Delta H_{\text {ads }}(n)}{R} \frac{1}{T}+C$. The variances calculated from the variance-covariance calculation were used as the weights in the ClausiusClapeyron linear fit. The last model used in this study is a virial-type equation, $\ln p=$ $\ln n+\frac{1}{T} \sum_{i=0}^{x} a_{i} n^{i}+\sum_{i=0}^{y} b_{i} n^{i}$. Unlike the abovementioned models, the virial analysis simultaneously fits the isothermal data for all temperatures. We have determined that $x=4$ and $y=1$ fits the data sufficiently with our data set. The isosteric enthalpy of adsorption is calculated as $\Delta H_{\text {ads }}(n)=R \sum_{i=0}^{x} a_{i} n^{i}$.

The various isotherm models were fit using the Nonlinearmodelfit function in Mathematica (which minimizes the sum of the squares of the residuals). Each fit is weighted by $1 /$ quantity adsorbed in order to ensure an appropriate model fit at the lowest pressures in order to achieve accurate isosteric enthalpy of adsorptions.

Figures presented in Section 6.2 to $\mathbf{6 . 5}$ include the best-fit model parameters, a plot of the experimental data and model with both linear-linear and log-log scales, the residuals of the fit, the $\mathrm{R} 2$ values for the Clausius-Clapeyron fit, the Clausius-Clapeyron fits with error bars, and the isosteric enthalpy of adsorption with error bars. 
Table S6.1: Summary of isosteric enthalpy of adsorption $(\mathrm{kJ} / \mathrm{mol})$.

$\begin{array}{lllllll}\text { GAS } & \mathbf{X} & \text { UNILAN } & \text { VIRIAL } & \text { SIPS } & \text { DSL } & \text { AVERAGE } \\ \mathrm{CO} & \mathrm{F} & 33.67 \pm 2.12 & 26.19 \pm 1.84 & 35.04 \pm 5.12 & 29.08 \pm 2.67 & \mathbf{3 1 . 0 0} \pm \mathbf{1 . 6 1} \\ & \mathrm{Cl} & 37.91 \pm 0.21 & 37.69 \pm 0.40 & 43.05 \pm 0.41 & 37.42 \pm 0.16 & \mathbf{3 9 . 0 2} \pm \mathbf{0 . 1 6} \\ & \mathrm{Br} & 40.18 \pm 0.55 & 46.09 \pm 1.16 & 50.97 \pm 2.57 & 41.00 \pm 0.86 & \mathbf{4 4 . 5 6} \pm \mathbf{0 . 7 5} \\ & \mathrm{OH} & 17.92 \pm 3.02 & 14.96 \pm 2.95 & 16.00 \pm 0.14 & 16.78 \pm 2.01 & \mathbf{1 6 . 4 2} \pm \mathbf{1 . 1 7} \\ \mathrm{C}_{2} \mathrm{H}_{4} & \mathrm{~F} & 27.21 \pm 0.96 & 32.14 \pm 0.49 & 43.20 \pm 1.04 & 29.38 \pm 0.51 & \mathbf{3 2 . 9 8} \pm \mathbf{0 . 4 0} \\ & \mathrm{Cl} & 21.28 \pm 0.63 & 22.81 \pm 0.16 & 22.99 \pm 0.49 & 22.17 \pm 0.53 & \mathbf{2 2 . 3 1} \pm \mathbf{0 . 2 4} \\ & \mathrm{Br} & 18.91 \pm 0.51 & 21.44 \pm 0.34 & 18.27 \pm 2.02 & 20.57 \pm 1.54 & \mathbf{1 9 . 8 0} \pm \mathbf{0 . 6 5} \\ & \mathrm{OH} & 16.96 \pm 1.31 & 22.56 \pm 0.39 & 29.60 \pm 3.98 & 23.78 \pm 1.80 & \mathbf{2 3 . 2 3} \pm \mathbf{1 . 1 4} \\ \mathrm{H} 2 & \mathrm{~F} & 6.86 \pm 0.00 & 7.11 \pm 0.08 & 7.78 \pm 0.01 & 6.60 \pm 0.00 & \mathbf{7 . 0 9} \pm \mathbf{0 . 0 2} \\ & \mathrm{Cl} & 7.42 \pm 0.00 & 7.46 \pm 0.03 & 8.73 \pm 0.00 & 6.91 \pm 0.00 & \mathbf{7 . 6 3} \pm \mathbf{0 . 0 1} \\ & \mathrm{Br} & 7.34 \pm 0.00 & 7.43 \pm 0.02 & 8.54 \pm 0.01 & 7.16 \pm 0.00 & \mathbf{7 . 6 2} \pm \mathbf{0 . 0 1} \\ & \mathrm{OH} & 6.22 \pm 0.01 & 6.73 \pm 0.03 & 8.66 \pm 0.01 & 6.40 \pm 0.00 & \mathbf{7 . 0 0} \pm \mathbf{0 . 0 1}\end{array}$

$\mathrm{a}_{-} \Delta \mathrm{H}_{\text {ads }}(\mathrm{kJ} / \mathrm{mol})$ determined at $0.01 \mathrm{mmol} / \mathrm{g}$ (this point was chosen rather than $0 \mathrm{mmol} / \mathrm{g}$ to prevent error resulting in extrapolation to ranges outside of measurement)

Table S6.2: Summary of mean absolute percentage error (MAPE) for each model (in \%). Expressed as $\frac{100}{N} \sum\left|\frac{q_{\text {meas }}-q_{\text {fit }}}{q_{\text {meas }}}\right|$ (for Unilan, Sips, DSL) and $\frac{100}{N} \sum\left|\frac{p_{\text {meas }}-p_{\text {fit }}}{p_{\text {meas }}}\right|$ (for virial).

\begin{tabular}{lllllll} 
GAS & $\mathbf{X}$ & UNILAN & VIRIAL & SIPS & DSL & AVERAGE \\
\hline Average & & $\mathbf{5 . 3 7}$ & $\mathbf{4 . 0 0}$ & $\mathbf{5 . 0 0}$ & $\mathbf{2 . 9 3}$ & $\mathbf{4 . 3 2}$ \\
$\mathrm{CO}$ & $\mathrm{F}$ & 7.68 & 13.65 & 7.17 & 6.90 & $\mathbf{8 . 8 5}$ \\
& $\mathrm{Cl}$ & 1.61 & 2.55 & 5.64 & 1.78 & $\mathbf{2 . 9 0}$ \\
& $\mathrm{Br}$ & 5.11 & 8.43 & 4.66 & 4.94 & $\mathbf{5 . 7 9}$ \\
& $\mathrm{OH}$ & 11.78 & 11.10 & 14.01 & 10.33 & $\mathbf{1 1 . 8 1}$ \\
$\mathrm{C}_{2} \mathrm{H}_{4}$ & $\mathrm{~F}$ & 4.61 & 2.22 & 4.26 & 1.19 & $\mathbf{3 . 0 7}$ \\
& $\mathrm{Cl}$ & 3.12 & 1.12 & 0.66 & 0.34 & $\mathbf{1 . 3 1}$ \\
& $\mathrm{Br}$ & 5.78 & 1.86 & 1.41 & 0.77 & $\mathbf{2 . 4 6}$ \\
& $\mathrm{OH}$ & 11.02 & 2.17 & 2.00 & 0.53 & $\mathbf{3 . 9 3}$ \\
$\mathrm{H} 2$ & $\mathrm{~F}$ & 2.09 & 2.31 & 6.85 & 2.61 & $\mathbf{3 . 4 7}$ \\
& $\mathrm{Cl}$ & 2.82 & 1.05 & 3.27 & 2.30 & $\mathbf{2 . 3 6}$ \\
& $\mathrm{Br}$ & 2.01 & 0.58 & 4.24 & 1.57 & $\mathbf{2 . 1 0}$ \\
& $\mathrm{OH}$ & 6.78 & 0.98 & 5.78 & 1.84 & $\mathbf{3 . 8 5}$
\end{tabular}


Figure S6.1: Summary of isosteric enthalpy of adsorption across models.
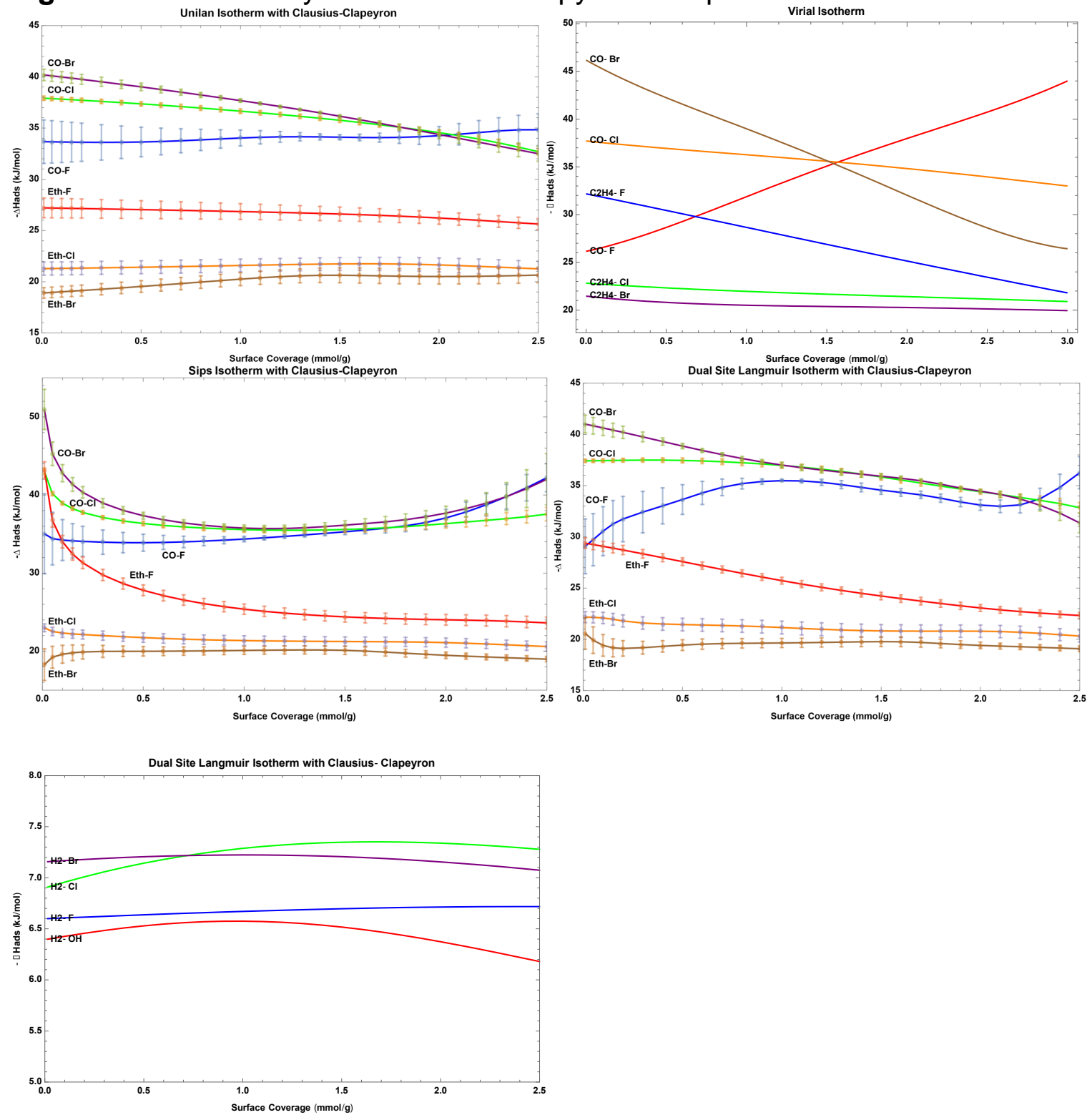


\section{Section 6.2. Unilan Models for Isosteric Enthalpy of Adsorption}

$n=\frac{U}{2 s} \ln \frac{1+p e^{s} / a}{1+p e^{-s} / a}$

$\mathrm{n}=$ quantity adsorbed $(\mathrm{mmol} / \mathrm{g})$

$\mathrm{p}=$ pressure $(\mathrm{kPa})$

$\mathrm{U}=$ maximum adsorption capacity

$\mathrm{a}=$ Unilan constant

$\mathrm{S}=$ difference between minimum and maximum adsorption energy

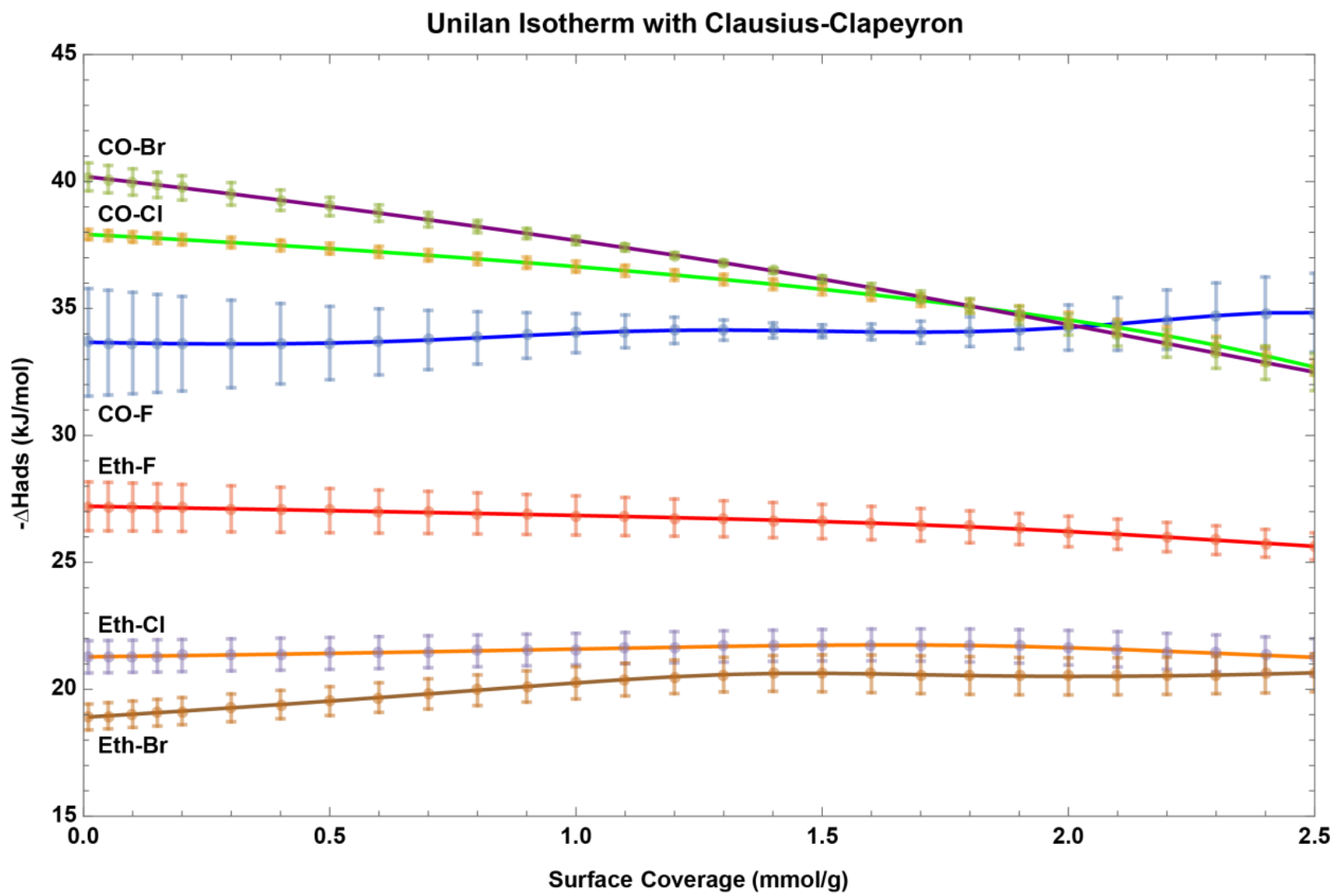

Summary of all Unilan/CC calculations. Error bars show one standard deviation.

$\mathrm{G}=\mathrm{CO}, \mathrm{X}=\mathrm{F}$

\begin{tabular}{|l|l|l|l|l|l|}
\hline & $283 \mathrm{~K}$ & $288 \mathrm{~K}$ & $293 \mathrm{~K}$ & $298 \mathrm{~K}$ & $308 \mathrm{~K}$ \\
\hline $\mathrm{U}$ & 5.63278 & 20.8899 & 21.5268 & 21.5705 & 20.6312 \\
\hline $\mathrm{s}$ & 3.15121 & 12.527 & 12.569 & 12.2529 & 11.846 \\
\hline $\mathrm{a}$ & 110.263 & $1.46024^{*} 10^{6}$ & $2.04597^{\star} 10^{6}$ & $2.00405^{\star} 10^{6}$ & $2.07604^{*} 10^{6}$ \\
\hline $\mathrm{R}^{2}$ & 0.99977 & 0.999813 & 0.999864 & 0.99969 & 0.999467 \\
\hline
\end{tabular}



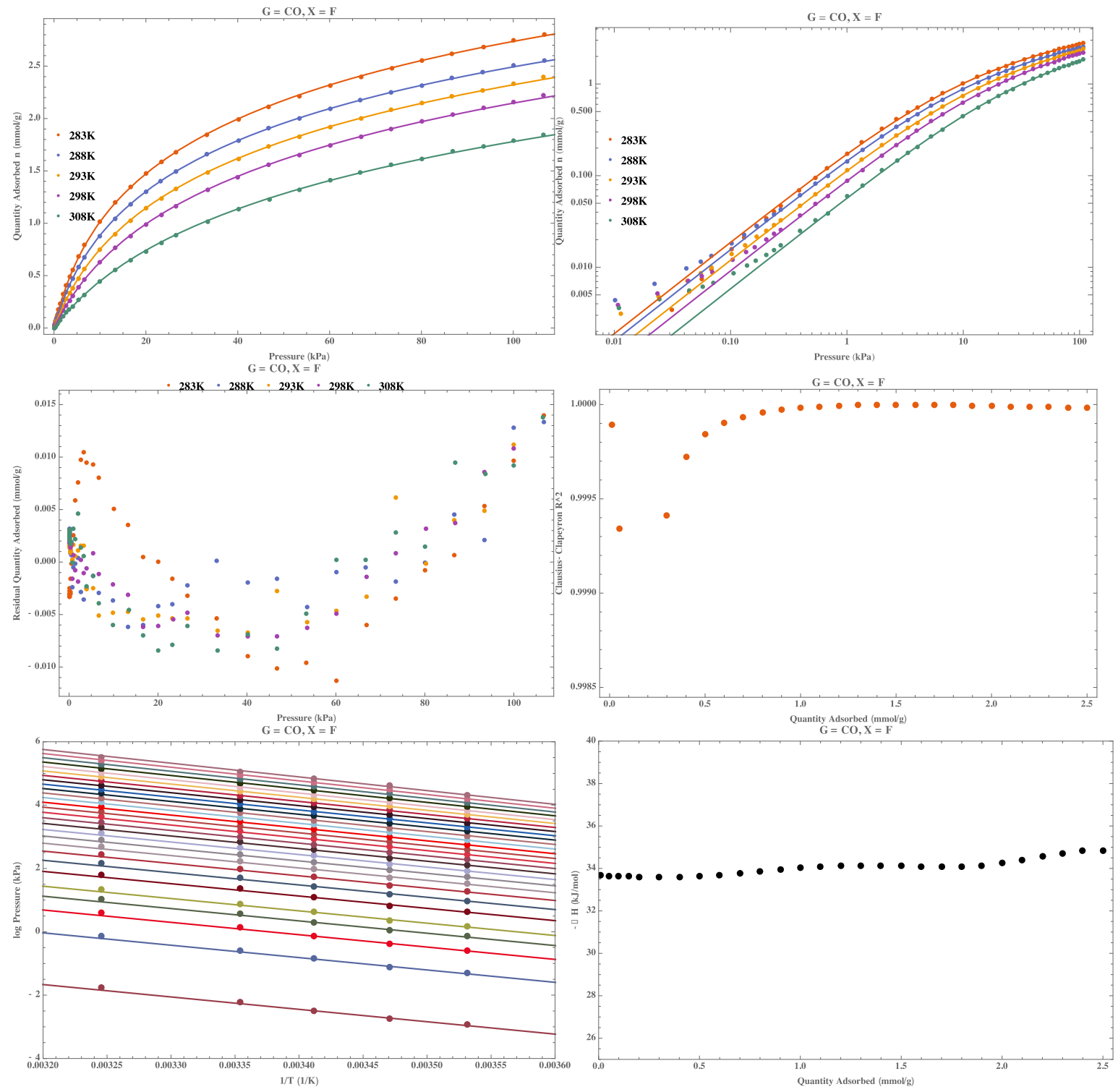

$\mathrm{G}=\mathrm{CO}, \mathrm{X}=\mathrm{Cl}$

\begin{tabular}{|l|l|l|l|l|l|}
\hline & $283 \mathrm{~K}$ & $288 \mathrm{~K}$ & $293 \mathrm{~K}$ & $298 \mathrm{~K}$ & $308 \mathrm{~K}$ \\
\hline $\mathrm{U}$ & 5.0222 & 5.19379 & 5.72677 & 7.27046 & 24.7394 \\
\hline $\mathrm{S}$ & 2.58975 & 2.62905 & 2.88274 & 3.67202 & 12.0629 \\
\hline $\mathrm{a}$ & 44.5242 & 62.6492 & 105.847 & 307.017 & $2.27772^{*} 10^{6}$ \\
\hline $\mathrm{R}^{2}$ & 0.999995 & 0.999995 & 0.999996 & 0.999967 & 0.999986 \\
\hline
\end{tabular}



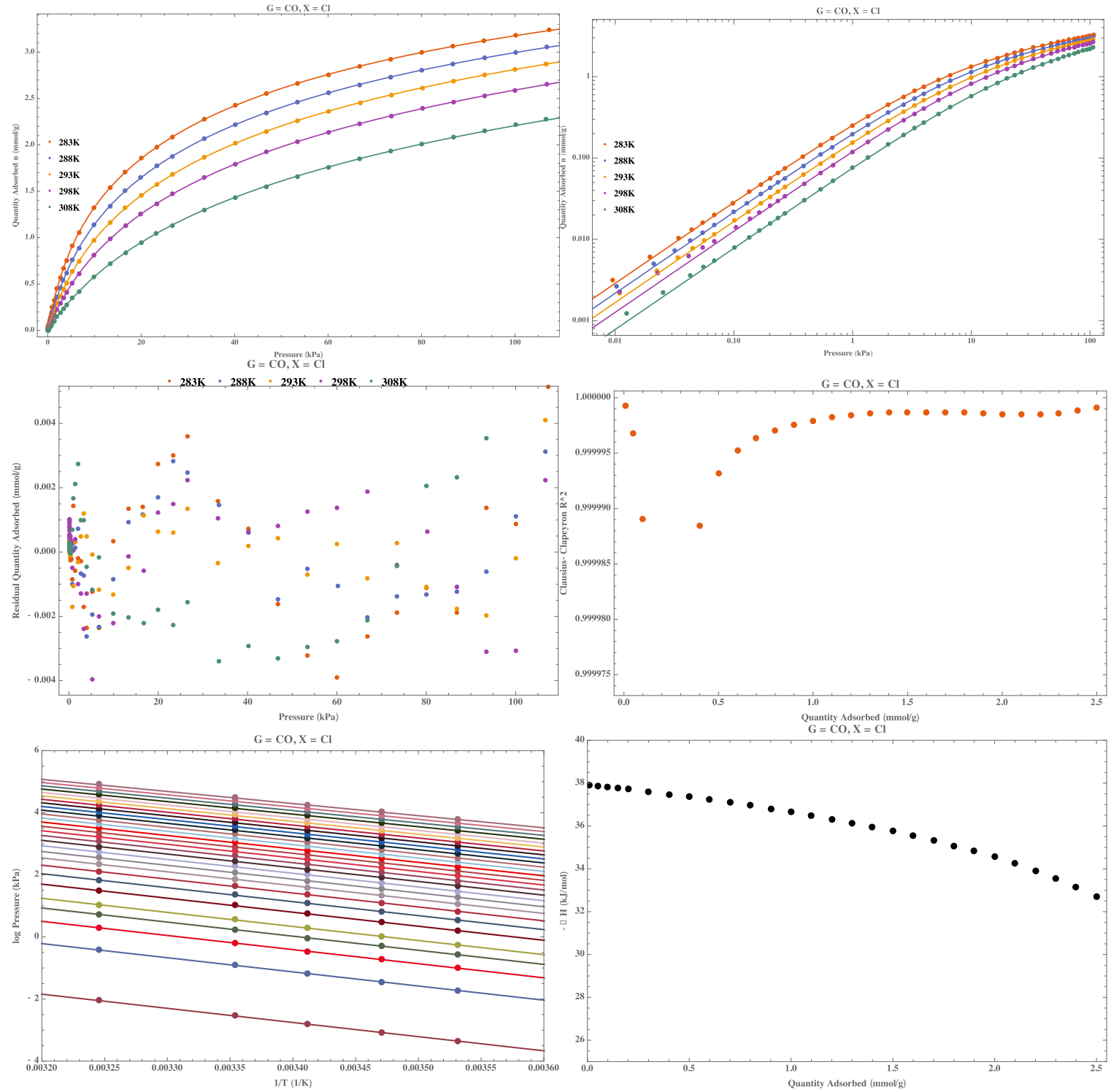

$\mathrm{G}=\mathrm{CO}, \mathrm{X}=\mathrm{Br}$

\begin{tabular}{|l|l|l|l|l|l|}
\hline & $283 \mathrm{~K}$ & $288 \mathrm{~K}$ & $293 \mathrm{~K}$ & $298 \mathrm{~K}$ & $308 \mathrm{~K}$ \\
\hline $\mathrm{U}$ & 17.8139 & 19.2549 & 18.099 & 18.813 & 17.7014 \\
\hline $\mathrm{S}$ & 12.5083 & 13.0374 & 11.8569 & 12.3436 & 11.0933 \\
\hline $\mathrm{a}$ & 724520 & $1.74737^{*} 10^{6}$ & 746012 & $1.54856^{*} 10^{6}$ & 794747 \\
\hline $\mathrm{R}^{2}$ & 0.99974 & 0.9996667 & 0.999947 & 0.99984 & 0.999984 \\
\hline
\end{tabular}



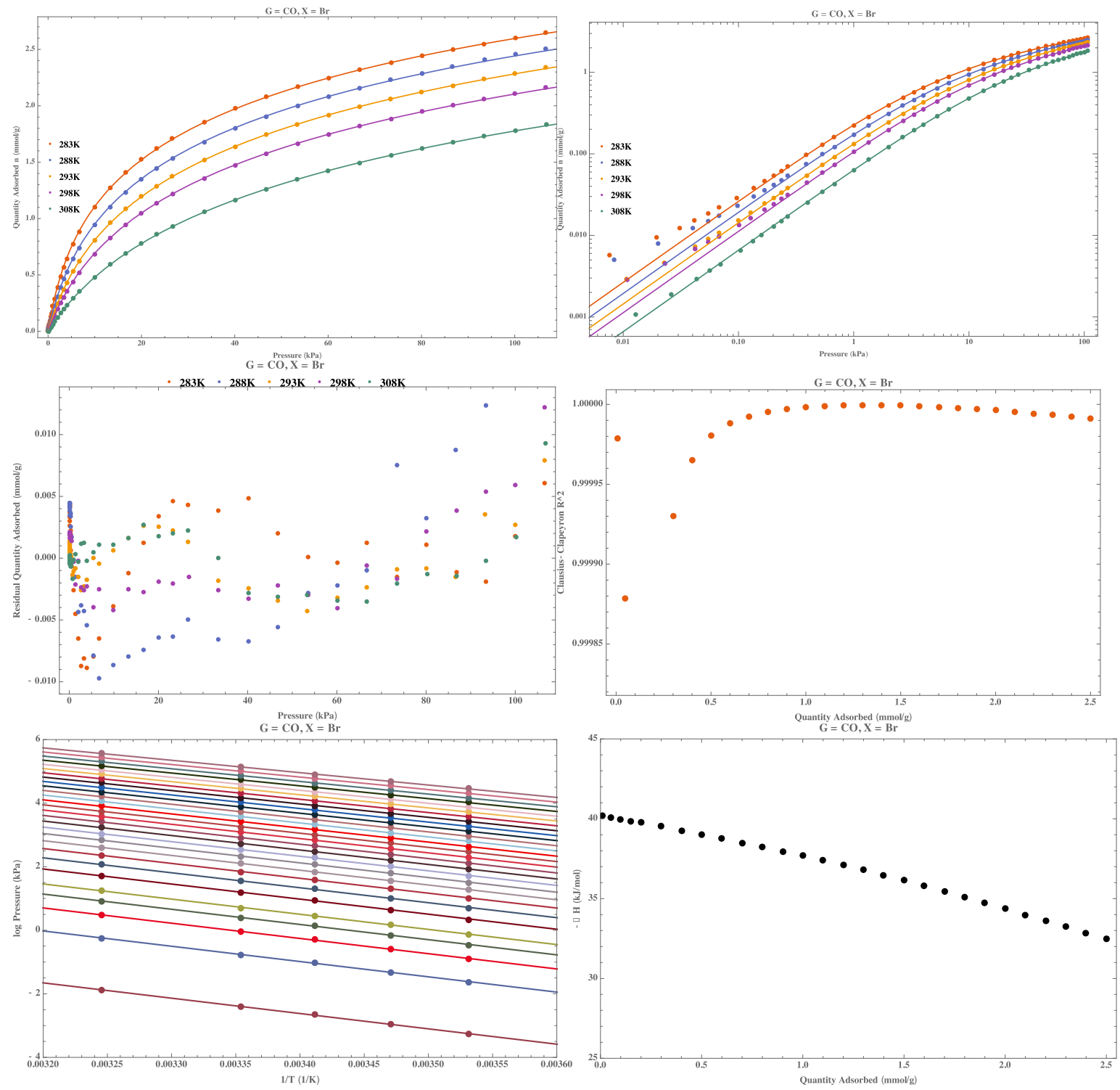

$\mathrm{G}=$ ethylene, $\mathrm{X}=\mathrm{F}$

\begin{tabular}{|l|l|l|l|l|l|}
\hline & $283 \mathrm{~K}$ & $288 \mathrm{~K}$ & $293 \mathrm{~K}$ & $298 \mathrm{~K}$ & $308 \mathrm{~K}$ \\
\hline $\mathrm{U}$ & 65.3984 & 64.2953 & 63.6257 & 63.9649 & 63.2723 \\
\hline $\mathrm{S}$ & 12.6045 & 12.482 & 12.3199 & 12.1315 & 11.1287 \\
\hline $\mathrm{a}$ & $6.41561^{*} 10^{6}$ & $6.72536^{*} 10^{6}$ & $6.82727^{\star} 10^{6}$ & $6.90939^{\star} 10^{6}$ & $4.0884^{*} 10^{6}$ \\
\hline $\mathrm{R}^{2}$ & 0.998638 & 0.99899 & 0.999373 & 0.999493 & 0.999799 \\
\hline
\end{tabular}



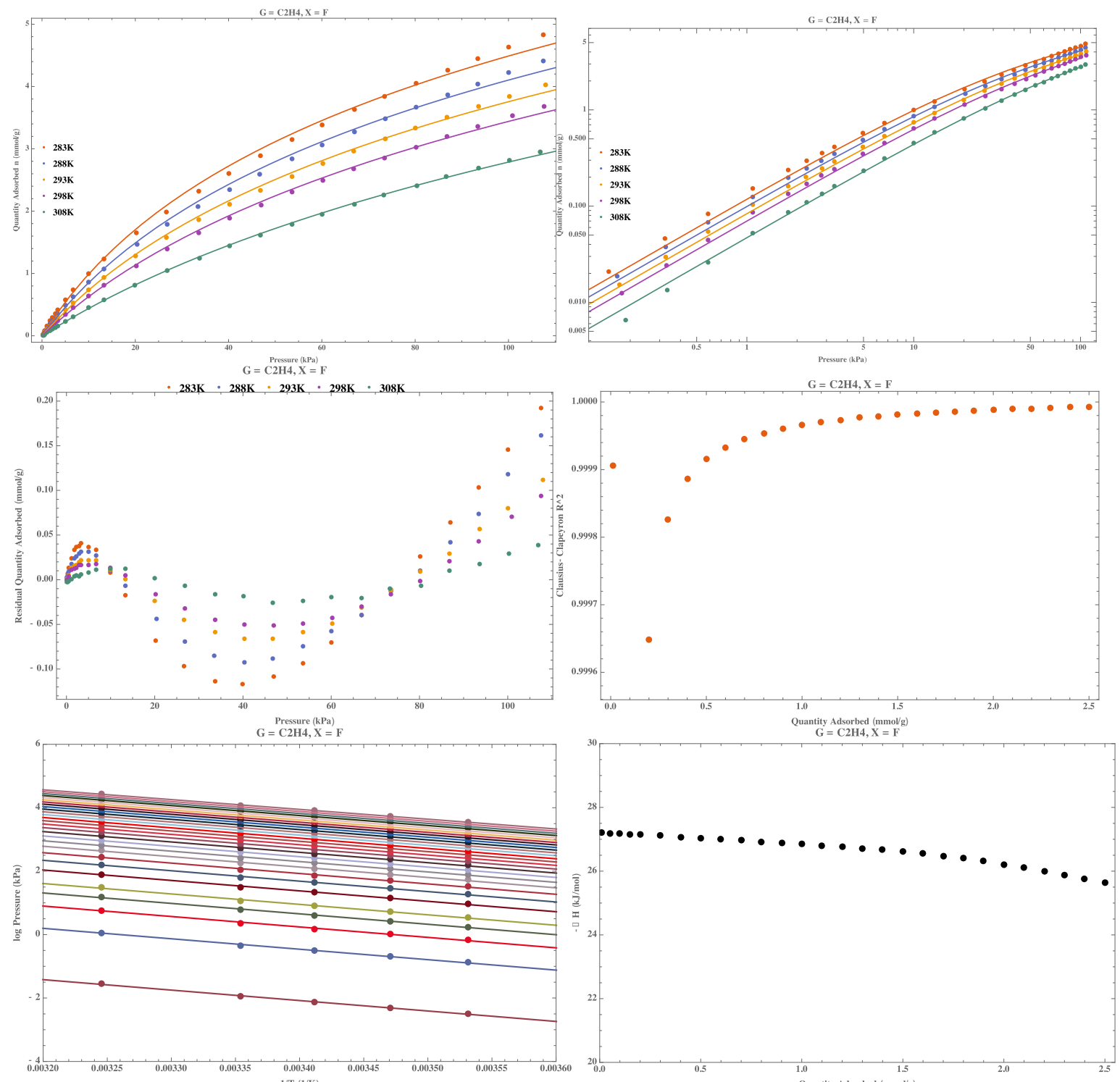

$\mathrm{G}=$ ethylene, $\mathrm{X}=\mathrm{Cl}$

\begin{tabular}{|l|l|l|l|l|l|}
\hline & $283 \mathrm{~K}$ & $288 \mathrm{~K}$ & $293 \mathrm{~K}$ & $298 \mathrm{~K}$ & $308 \mathrm{~K}$ \\
\hline $\mathrm{U}$ & 133.335 & 130.957 & 126.82 & 126.815 & 116.632 \\
\hline $\mathrm{s}$ & 10.7864 & 10.6319 & 10.3772 & 10.2796 & 10.0138 \\
\hline $\mathrm{a}$ & $4.63904^{\star} 10^{6}$ & $4.55355^{\star} 10^{6}$ & $4.03451^{\star} 10^{6}$ & $4.35625^{\star} 10^{6}$ & $4.2323^{\star} 10^{6}$ \\
\hline $\mathrm{R}^{2}$ & 0.999788 & 0.999805 & 0.999806 & 0.999806 & 0.999819 \\
\hline
\end{tabular}



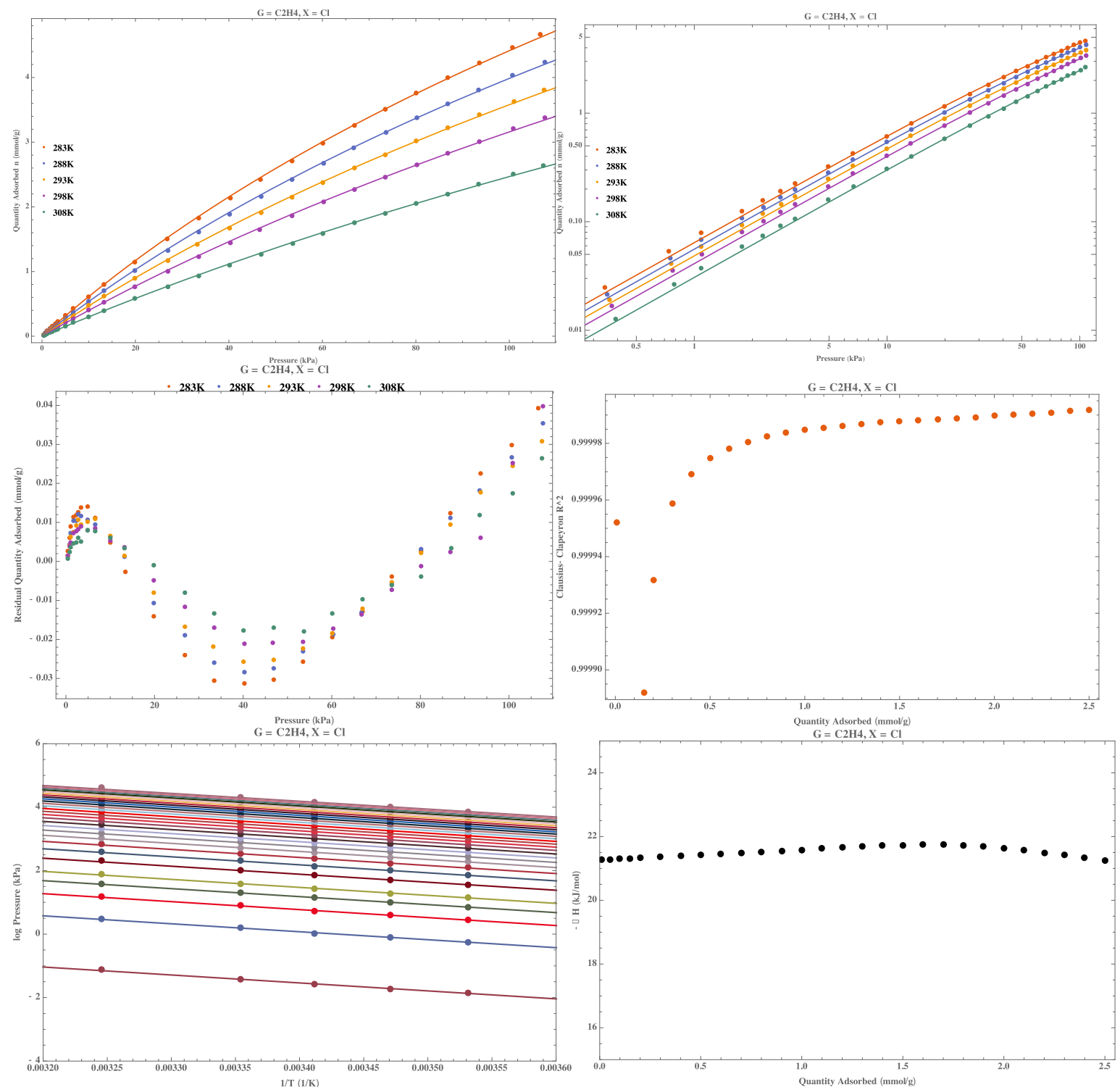

$\mathrm{G}=$ ethylene, $\mathrm{X}=\mathrm{Br}$

\begin{tabular}{|l|l|l|l|l|l|}
\hline & $283 \mathrm{~K}$ & $288 \mathrm{~K}$ & $293 \mathrm{~K}$ & $298 \mathrm{~K}$ & $308 \mathrm{~K}$ \\
\hline $\mathrm{U}$ & 142.249 & 136.116 & 123.941 & 110.549 & 90.9118 \\
\hline $\mathrm{S}$ & 10.2099 & 10.1586 & 10.1083 & 10.1124 & 10.0267 \\
\hline $\mathrm{a}$ & $4.19987^{\star} 10^{6}$ & $4.37677^{\star} 10^{6}$ & $4.33957^{\star} 10^{6}$ & $4.38547^{\star} 10^{6}$ & $4.43476^{\star} 10^{6}$ \\
\hline $\mathrm{R}^{2}$ & 0.999489 & 0.999276 & 0.999322 & 0.999205 & 0.999373 \\
\hline
\end{tabular}



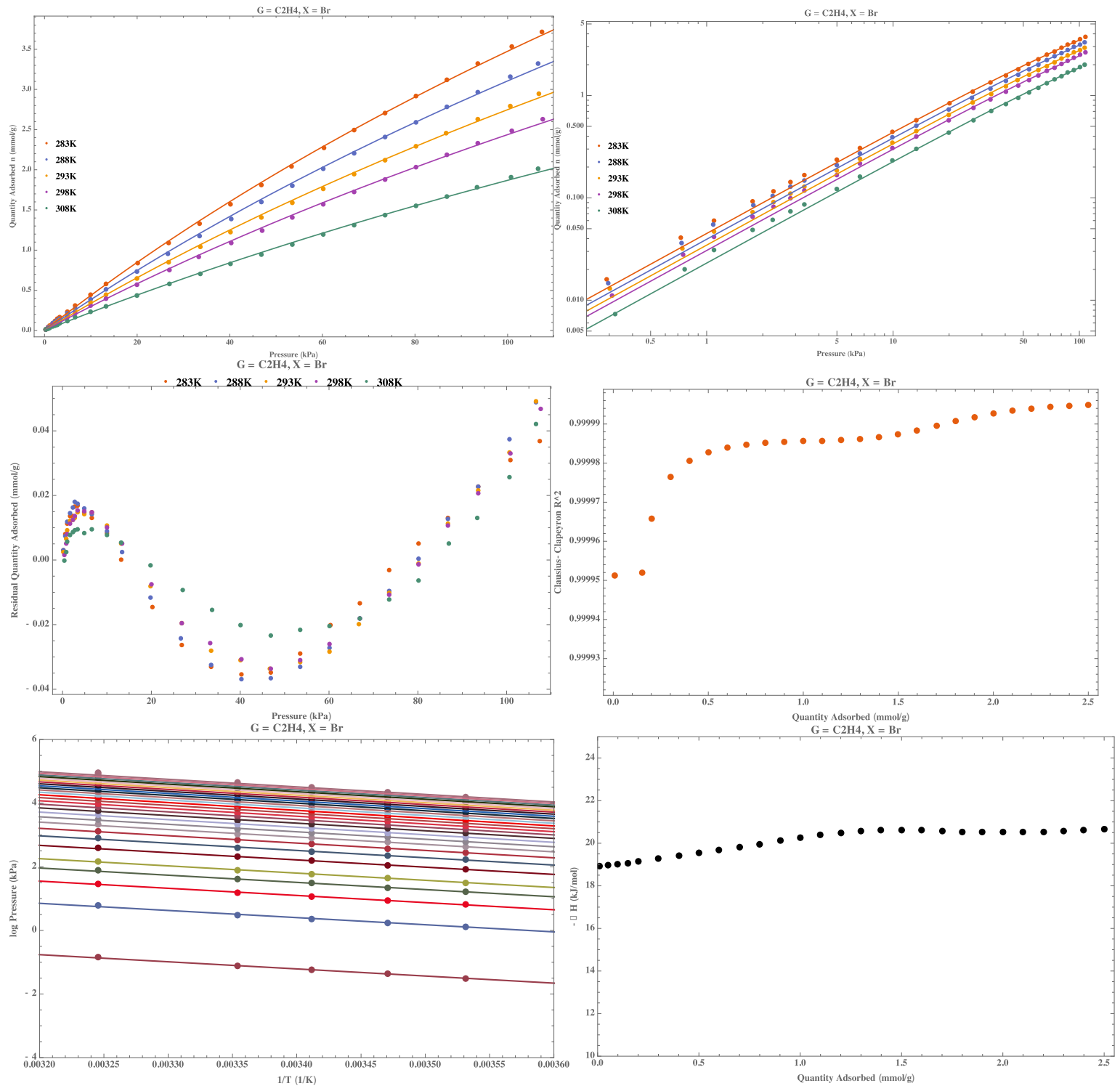


\section{Section 6.3. Virial Model for Isosteric Enthalpy of Adsorption}

$\ln p=\ln n+\frac{1}{T} \sum_{i=0}^{4} a_{i} n^{i}+\sum_{i=0}^{1} b_{i} n^{i}$

$\Delta H_{\text {ads }}(n)=R \sum_{i=0}^{4} a_{i} n^{i}$

$\mathrm{n}=$ quantity adsorbed $(\mathrm{mmol} / \mathrm{g})$

$\mathrm{p}=$ pressure $(\mathrm{kPa})$

$\mathrm{T}=$ temperature $(\mathrm{K})$

$a_{i}, b_{i}=$ virial coefficients $\left(K / \mathrm{mol}^{i}\right)$

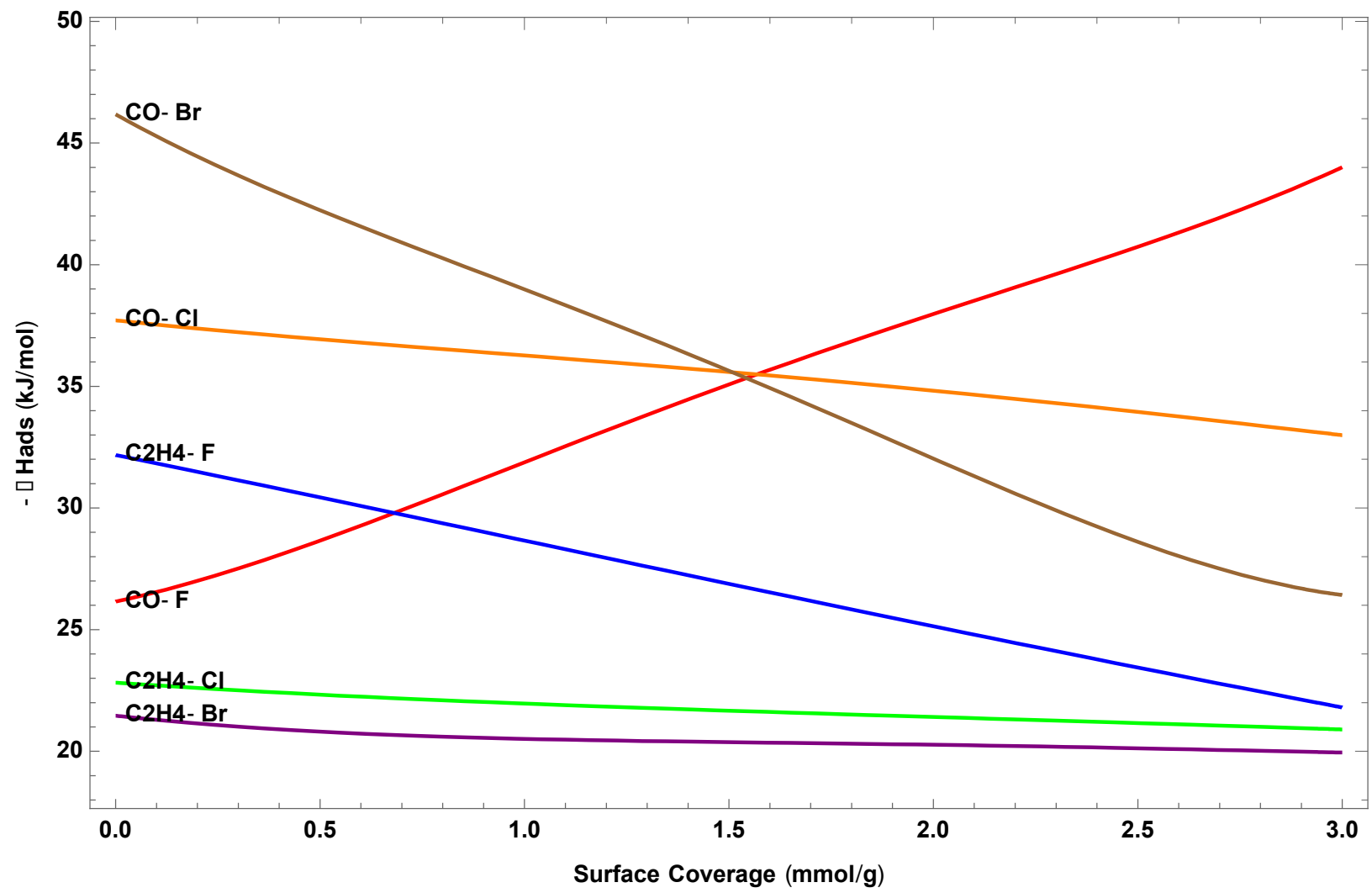

Summary of Virial Isotherm Fits. Dotted lines are one standard deviation.

$\mathrm{G}=\mathrm{CO}, \mathrm{X}=\mathrm{F}$

\begin{tabular}{|l|l|l|}
\hline & Estimate & Standard Error \\
\hline$a_{0}$ & -3145.29 & 222.67 \\
\hline$a_{1}$ & -430.24 & 246.012 \\
\hline$a_{2}$ & -450.004 & 141.225 \\
\hline$a_{3}$ & 228.036 & 84.4302 \\
\hline$a_{4}$ & -36.5873 & 16.0848 \\
\hline$b_{0}$ & 12.6571 & 0.754852 \\
\hline$b_{1}$ & 3.21696 & 0.792216 \\
\hline
\end{tabular}




$$
R^{2}=0.994755
$$
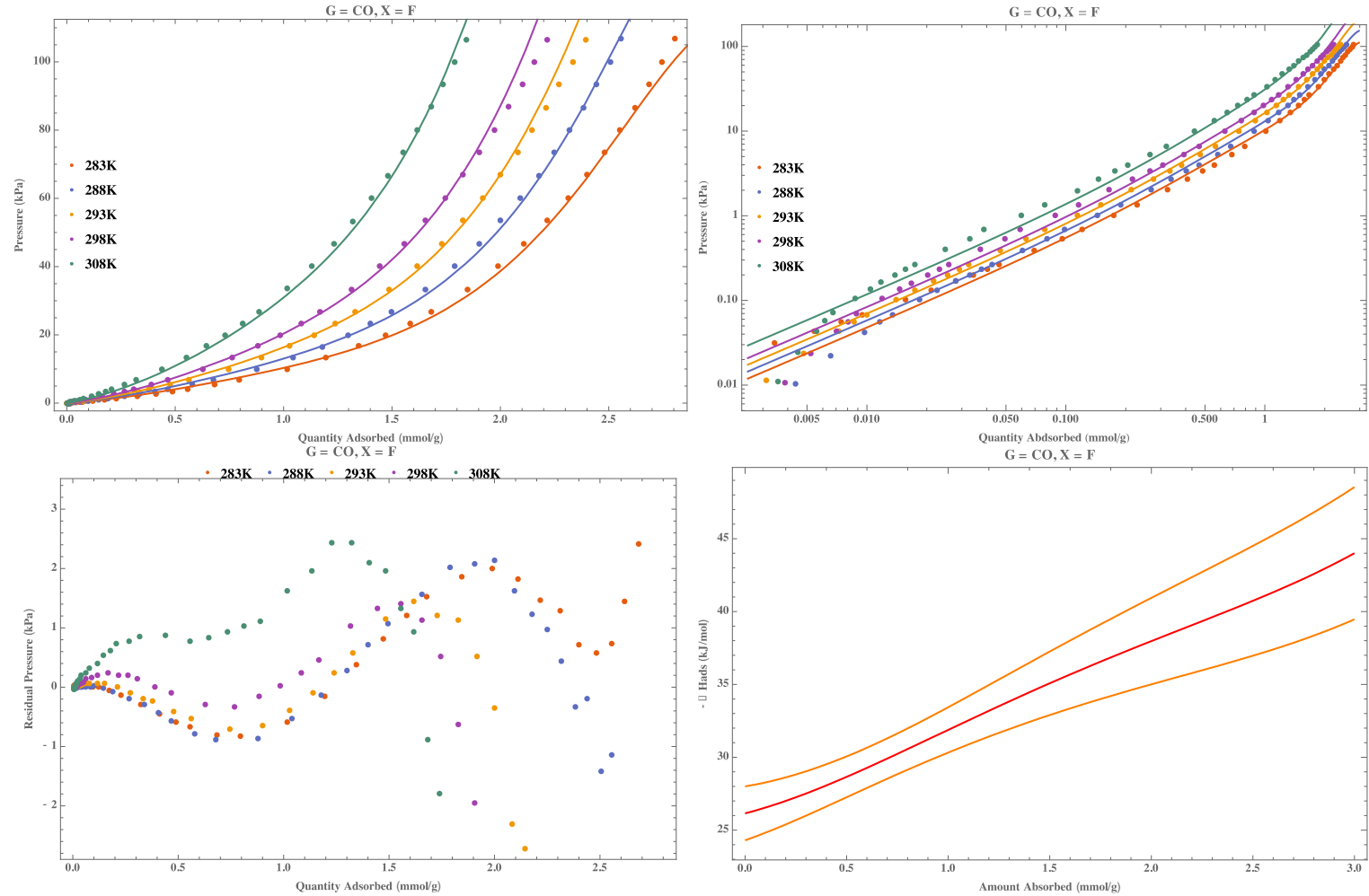

$\mathrm{G}=\mathrm{CO}, \mathrm{X}=\mathrm{Cl}$

\begin{tabular}{|l|l|l|}
\hline & Estimate & Standard Error \\
\hline$a_{0}$ & -4535.79 & 48.3746 \\
\hline$a_{1}$ & 214.899 & 44.1429 \\
\hline$a_{2}$ & -70.4058 & 23.1363 \\
\hline$a_{3}$ & 33.5374 & 11.9493 \\
\hline$a_{4}$ & -4.30791 & 1.96507 \\
\hline$b_{0}$ & 17.2152 & 0.16415 \\
\hline$b_{1}$ & 0.0257865 & 0.140197 \\
\hline
\end{tabular}

$R^{2}=0.999749$ 

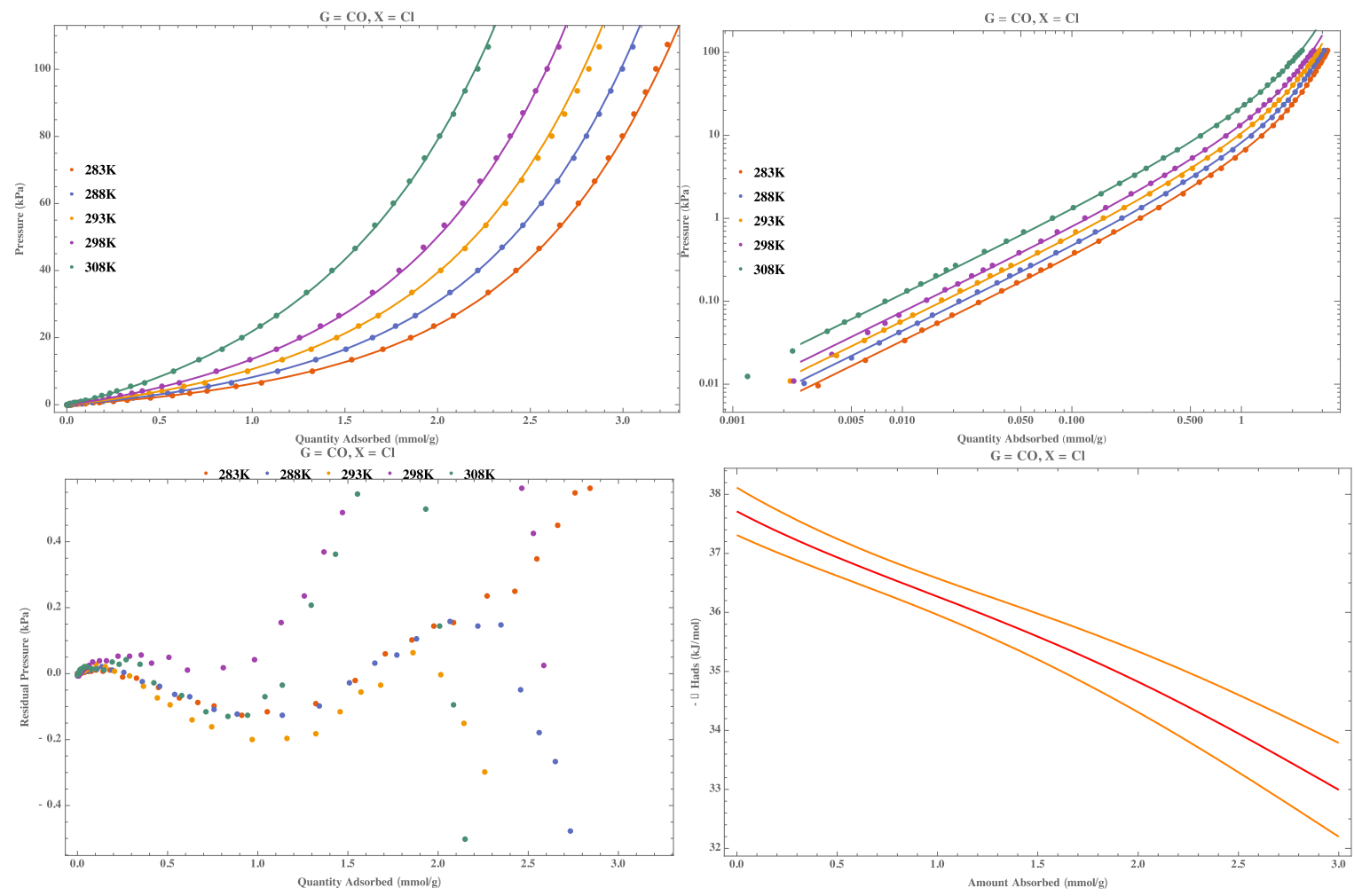

$\mathrm{G}=\mathrm{CO}, \mathrm{X}=\mathrm{Br}$

\begin{tabular}{|l|l|l|}
\hline & Estimate & Standard Error \\
\hline$a_{0}$ & -5554.49 & 140.656 \\
\hline$a_{1}$ & 1132.74 & 157.623 \\
\hline$a_{2}$ & -492.002 & 100.465 \\
\hline$a_{3}$ & 274.374 & 63.2769 \\
\hline$a_{4}$ & -49.405 & 12.7042 \\
\hline$b_{0}$ & 20.6528 & 0.476755 \\
\hline$b_{1}$ & -1.98746 & 0.498593 \\
\hline
\end{tabular}

$R^{2}=0.997923$ 

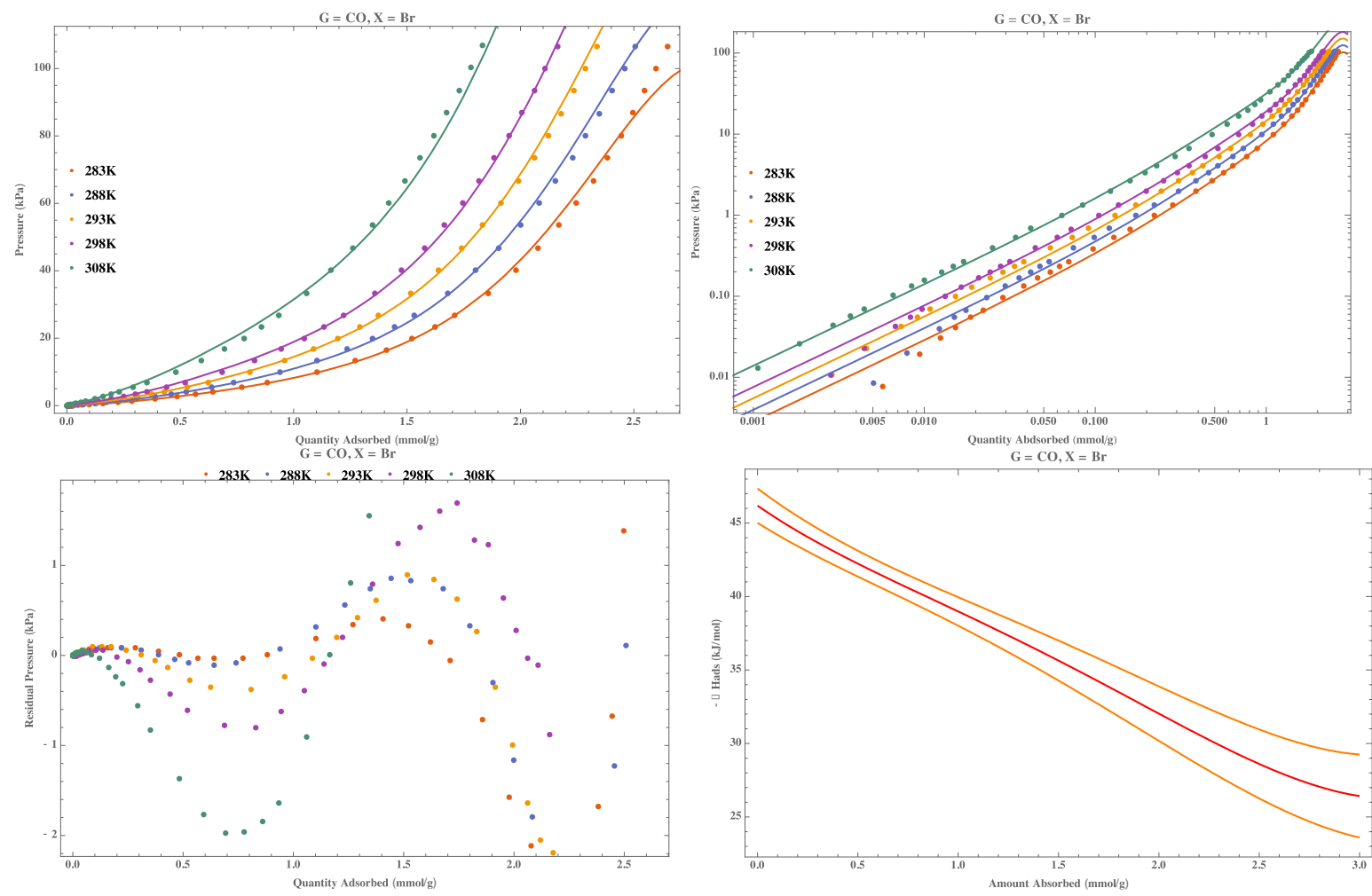

$\mathrm{G}=\mathrm{C} 2 \mathrm{H} 4, \mathrm{X}=\mathrm{F}$

\begin{tabular}{|l|l|l|}
\hline & Estimate & Standard Error \\
\hline$a_{0}$ & -3869.99 & 59.6324 \\
\hline$a_{1}$ & 410.376 & 36.8163 \\
\hline$a_{2}$ & 20.9295 & 11.6294 \\
\hline$a_{3}$ & -9.05943 & 3.93495 \\
\hline$a_{4}$ & 0.903879 & 0.429069 \\
\hline$b_{0}$ & 15.5967 & 0.201573 \\
\hline$b_{1}$ & -1.1733 & 0.11743 \\
\hline
\end{tabular}

$R^{2}=0.999828$ 

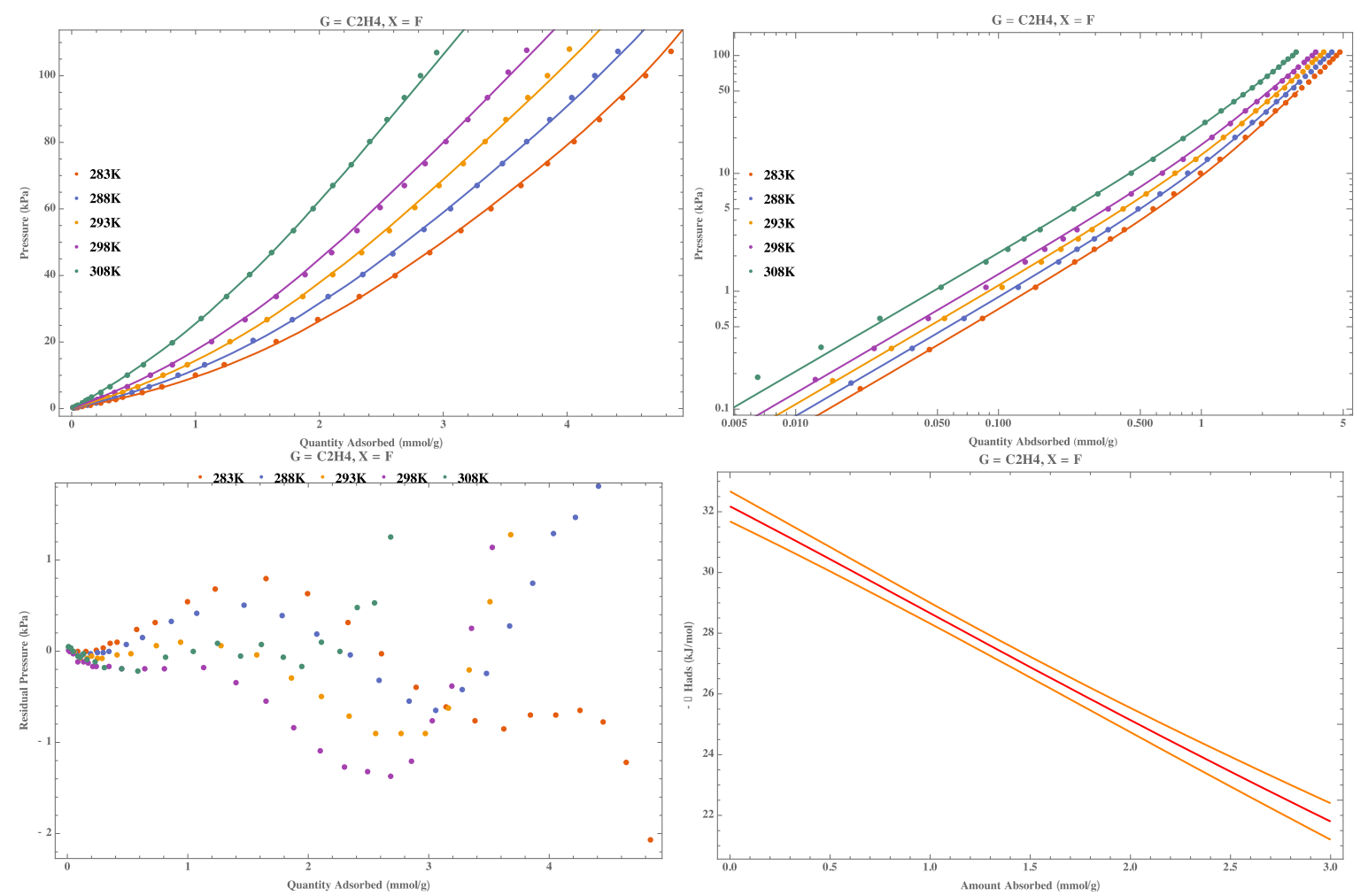

$\mathrm{G}=\mathrm{C} 2 \mathrm{H} 4, \mathrm{X}=\mathrm{Cl}$

\begin{tabular}{|l|l|l|}
\hline & Estimate & Standard Error \\
\hline $\mathrm{a}_{0}$ & -2744.61 & 19.6555 \\
\hline $\mathrm{a}_{1}$ & 138.707 & 13.6658 \\
\hline $\mathrm{a}_{2}$ & -45.7346 & 4.35165 \\
\hline $\mathrm{a}_{3}$ & 11.3473 & 1.53692 \\
\hline $\mathrm{a}_{4}$ & -0.983806 & 0.174077 \\
\hline $\mathrm{b}_{0}$ & 12.2969 & 0.0666057 \\
\hline $\mathrm{b}_{1}$ & -0.14488 & 0.0442618 \\
\hline
\end{tabular}

$R^{2}=0.999981$ 

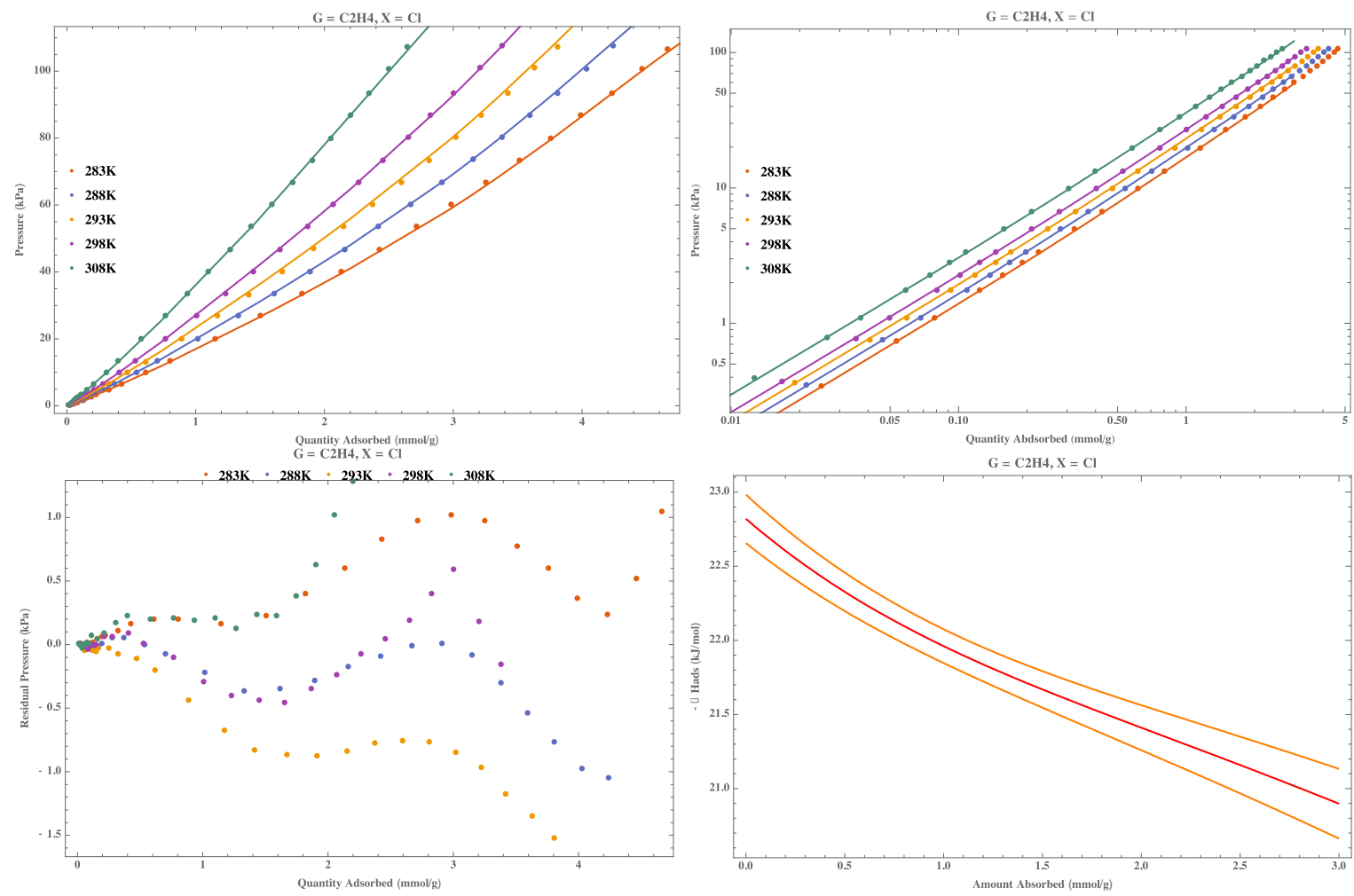

$\mathrm{G}=\mathrm{C} 2 \mathrm{H} 4, \mathrm{X}=\mathrm{Br}$

\begin{tabular}{|l|l|l|}
\hline & Estimate & Standard Error \\
\hline $\mathrm{a}_{0}$ & -2581.4 & 40.8822 \\
\hline $\mathrm{a}_{1}$ & 222.15 & 37.1675 \\
\hline $\mathrm{a}_{2}$ & -150.99 & 14.3773 \\
\hline $\mathrm{a}_{3}$ & 48.9921 & 6.141441 \\
\hline $\mathrm{a}_{4}$ & -5.53159 & 0.915535 \\
\hline $\mathrm{b}_{0}$ & 11.9703 & 0.138634 \\
\hline $\mathrm{b}_{1}$ & -0.058865 & 0.121385 \\
\hline
\end{tabular}

$R^{2}=0.999918$ 

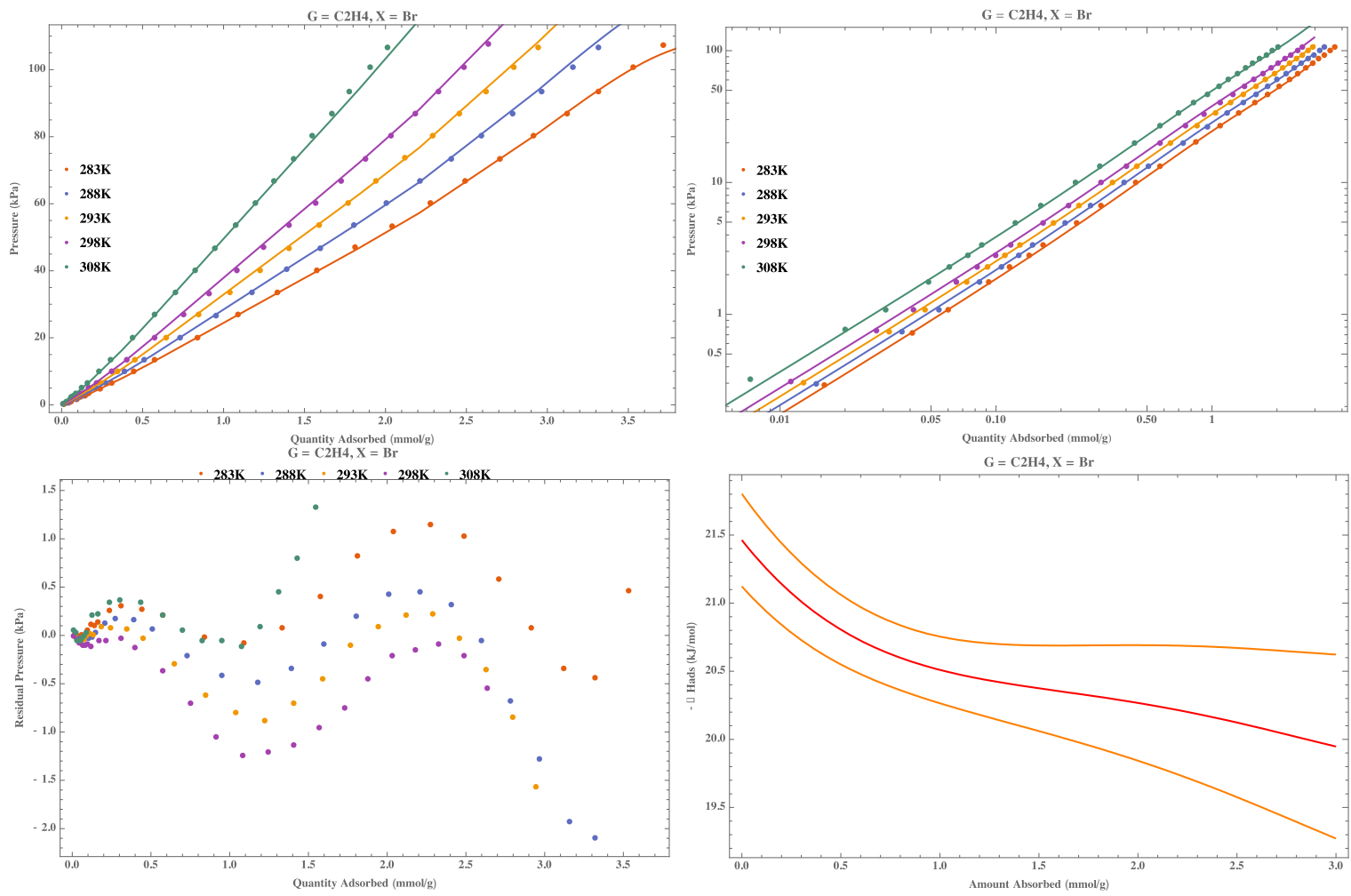


\section{Section 6.4. Sips (Langmuir-Freundlich) Models for Isosteric Enthalpy of Adsorption}

$n=\frac{U a p^{s}}{1+a p^{s}}$

$\mathrm{n}=$ quantity adsorbed $(\mathrm{mmol} / \mathrm{g})$

$\mathrm{p}=$ pressure $(\mathrm{kPa})$

$U=$ maximum adsorption capacity

$\mathrm{a}=$ Sips constant

$\mathrm{S}=$ heterogeneity factor

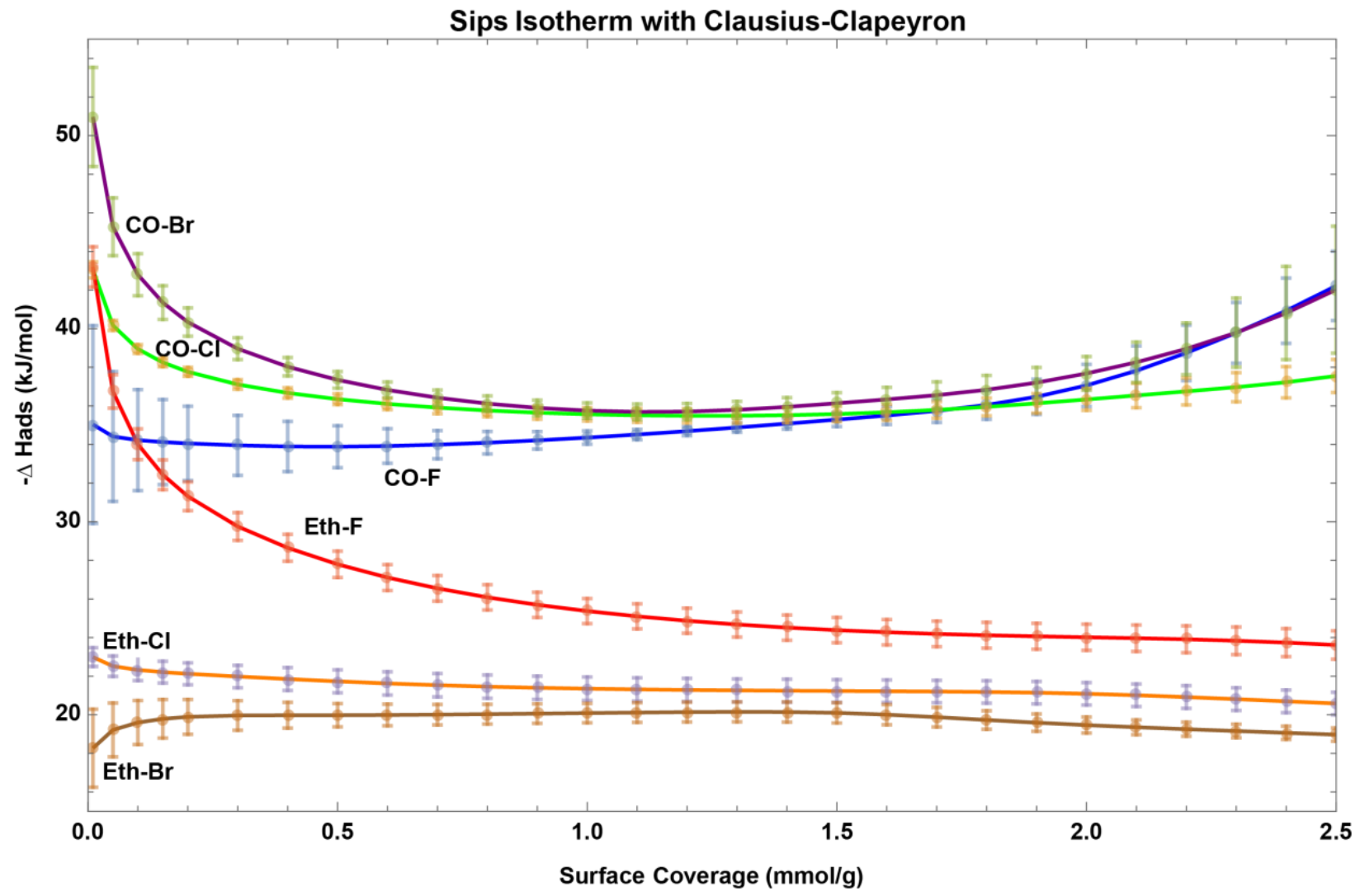

Summary of all Sips/CC calculations. Error bars show one standard deviation.

$\mathrm{G}=\mathrm{CO}, \mathrm{X}=\mathrm{F}$

\begin{tabular}{|l|l|l|l|l|l|}
\hline & $283 \mathrm{~K}$ & $288 \mathrm{~K}$ & $293 \mathrm{~K}$ & $298 \mathrm{~K}$ & $308 \mathrm{~K}$ \\
\hline $\mathrm{U}$ & 3.44571 & 3.41418 & 3.34023 & 3.30429 & 3.14974 \\
\hline $\mathrm{s}$ & 0.917805 & 0.880539 & 0.894853 & 0.902536 & 0.907754 \\
\hline $\mathrm{a}$ & 0.0497797 & 0.04447 & 0.0356671 & 0.0284464 & 0.0198512 \\
\hline
\end{tabular}




\begin{tabular}{|l|l|l|l|l|l|}
\hline $\mathrm{R}^{2}$ & 0.998692 & 0.999592 & 0.99966 & 0.999667 & 0.999712 \\
\hline
\end{tabular}
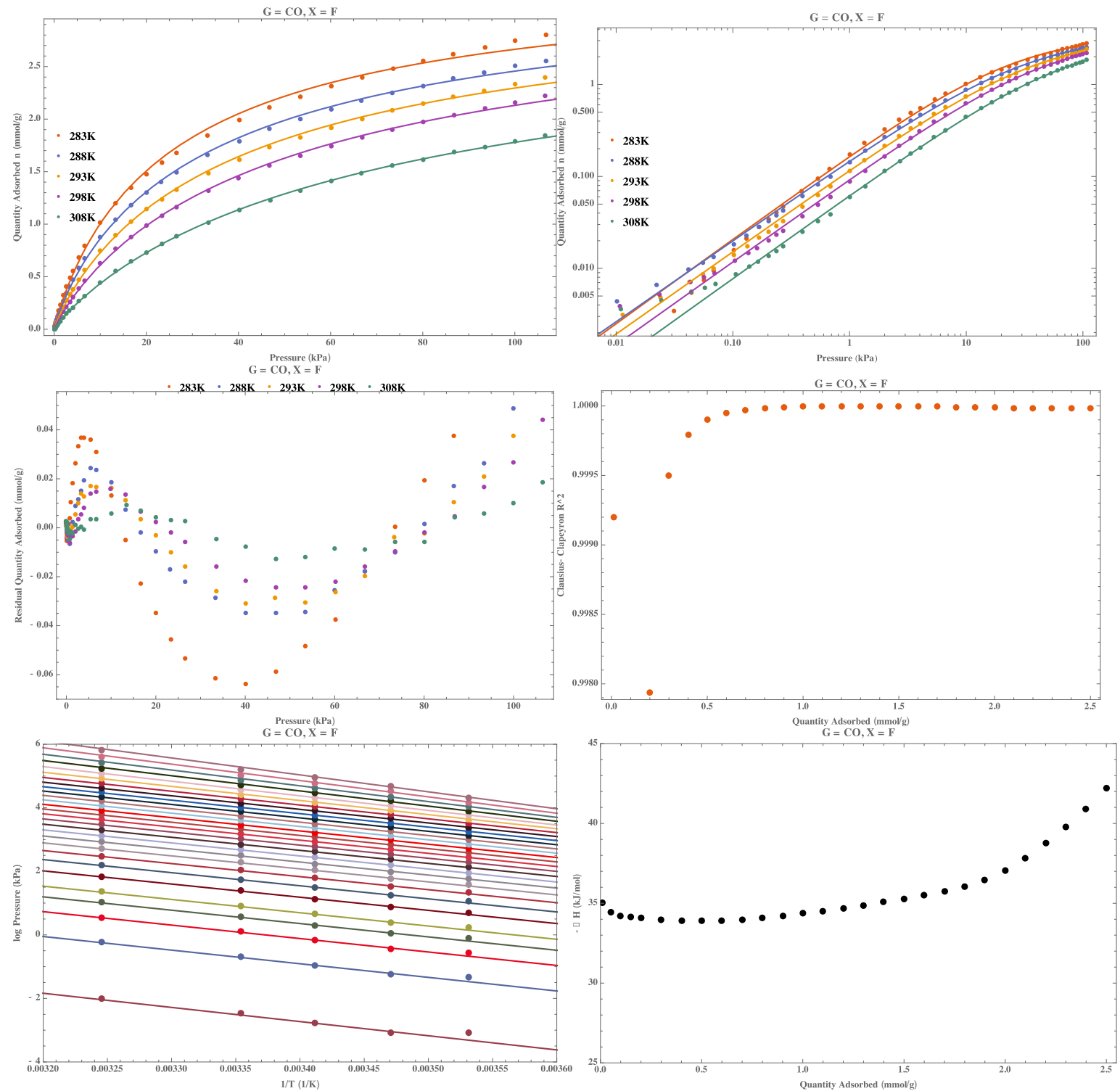

$\mathrm{G}=\mathrm{CO}, \mathrm{X}=\mathrm{Cl}$

\begin{tabular}{|l|l|l|l|l|l|}
\hline & $283 \mathrm{~K}$ & $288 \mathrm{~K}$ & $293 \mathrm{~K}$ & $298 \mathrm{~K}$ & $308 \mathrm{~K}$ \\
\hline $\mathrm{U}$ & 3.90009 & 3.82286 & 3.7482 & 3.67129 & 3.48608 \\
\hline $\mathrm{S}$ & 0.88546 & 0.898632 & 0.910116 & 0.914805 & 0.936993 \\
\hline $\mathrm{a}$ & 0.0664391 & 0.0527782 & 0.0423251 & 0.0337662 & 0.0224625 \\
\hline $\mathrm{R}^{2}$ & 0.999395 & 0.999513 & 0.99957 & 0.999744 & 0.999795 \\
\hline
\end{tabular}



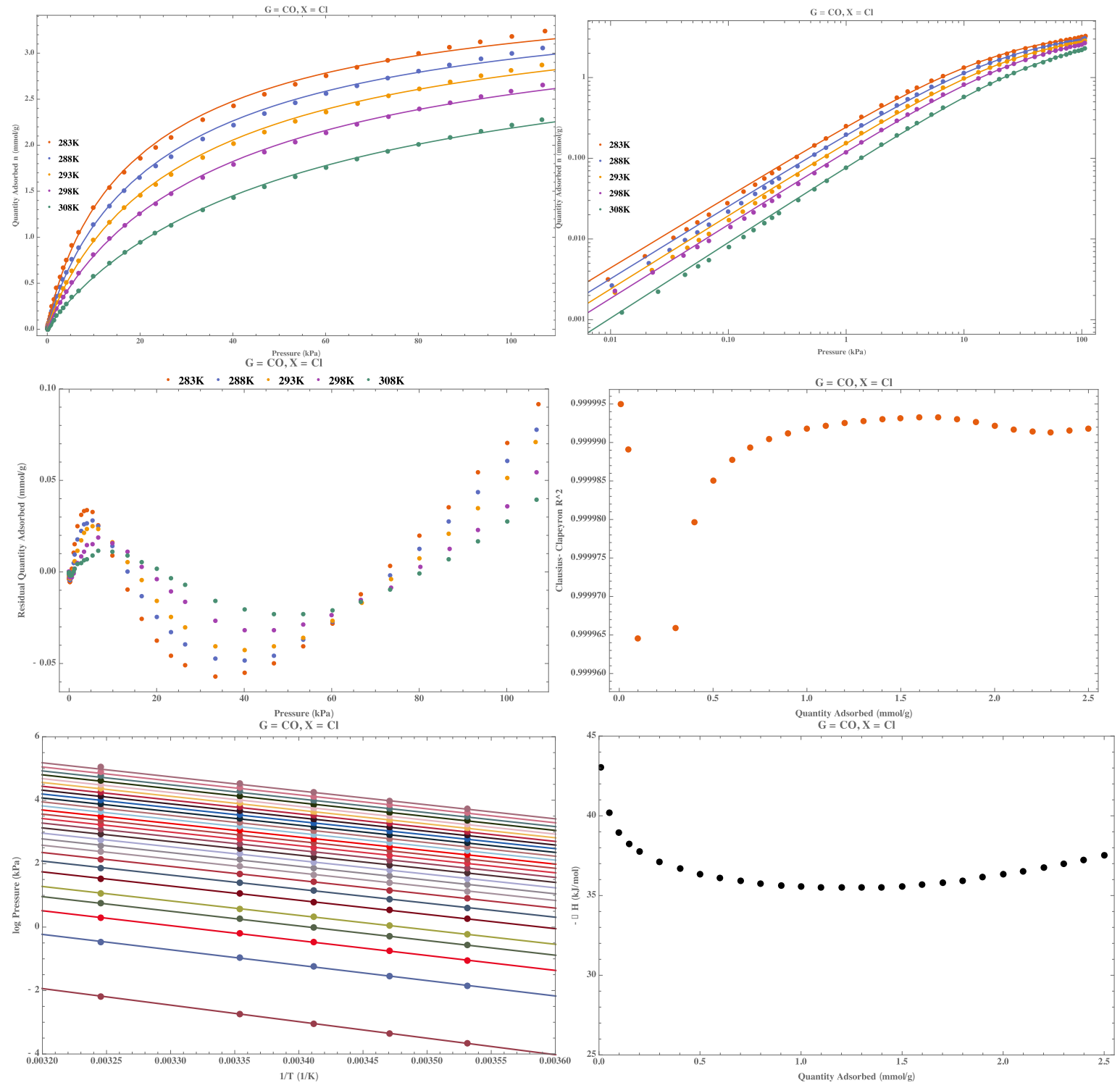

$\mathrm{G}=\mathrm{CO}, \mathrm{X}=\mathrm{Br}$

\begin{tabular}{|l|l|l|l|l|l|}
\hline & $283 \mathrm{~K}$ & $288 \mathrm{~K}$ & $293 \mathrm{~K}$ & $298 \mathrm{~K}$ & $308 \mathrm{~K}$ \\
\hline $\mathrm{U}$ & 3.27187 & 3.27314 & 3.05511 & 2.99641 & 2.69418 \\
\hline $\mathrm{s}$ & 0.84281 & 0.849174 & 0.893861 & 0.892136 & 0.943709 \\
\hline $\mathrm{a}$ & 0.0719193 & 0.0558379 & 0.0448456 & 0.0372138 & 0.0241213 \\
\hline $\mathrm{R}^{2}$ & 0.999502 & 0.999556 & 0.999552 & 0.999717 & 0.999704 \\
\hline
\end{tabular}



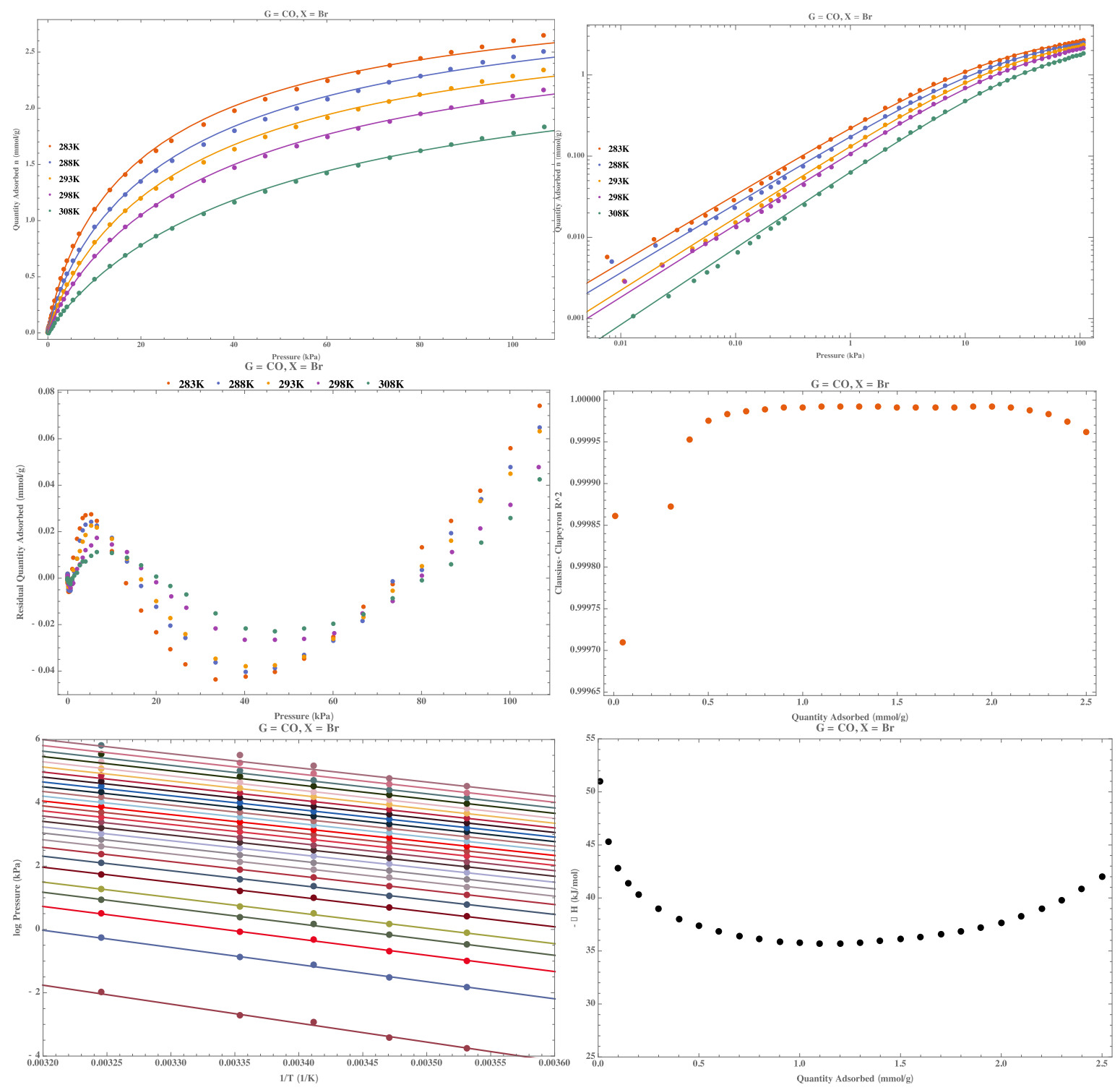

$\mathrm{G}=\mathrm{C} 2 \mathrm{H} 4, \mathrm{X}=\mathrm{F}$

\begin{tabular}{|l|l|l|l|l|l|}
\hline & $283 \mathrm{~K}$ & $288 \mathrm{~K}$ & $293 \mathrm{~K}$ & $298 \mathrm{~K}$ & $308 \mathrm{~K}$ \\
\hline $\mathrm{U}$ & 12.3524 & 11.3064 & 10.1453 & 9.86665 & 8.21607 \\
\hline $\mathrm{a}$ & 0.844591 & 0.866797 & 0.893866 & 0.908688 & 0.964091 \\
\hline $\mathrm{R}^{2}$ & 0.0120794 & 0.0108159 & 0.00977922 & 0.00828876 & 0.00608007 \\
\hline
\end{tabular}



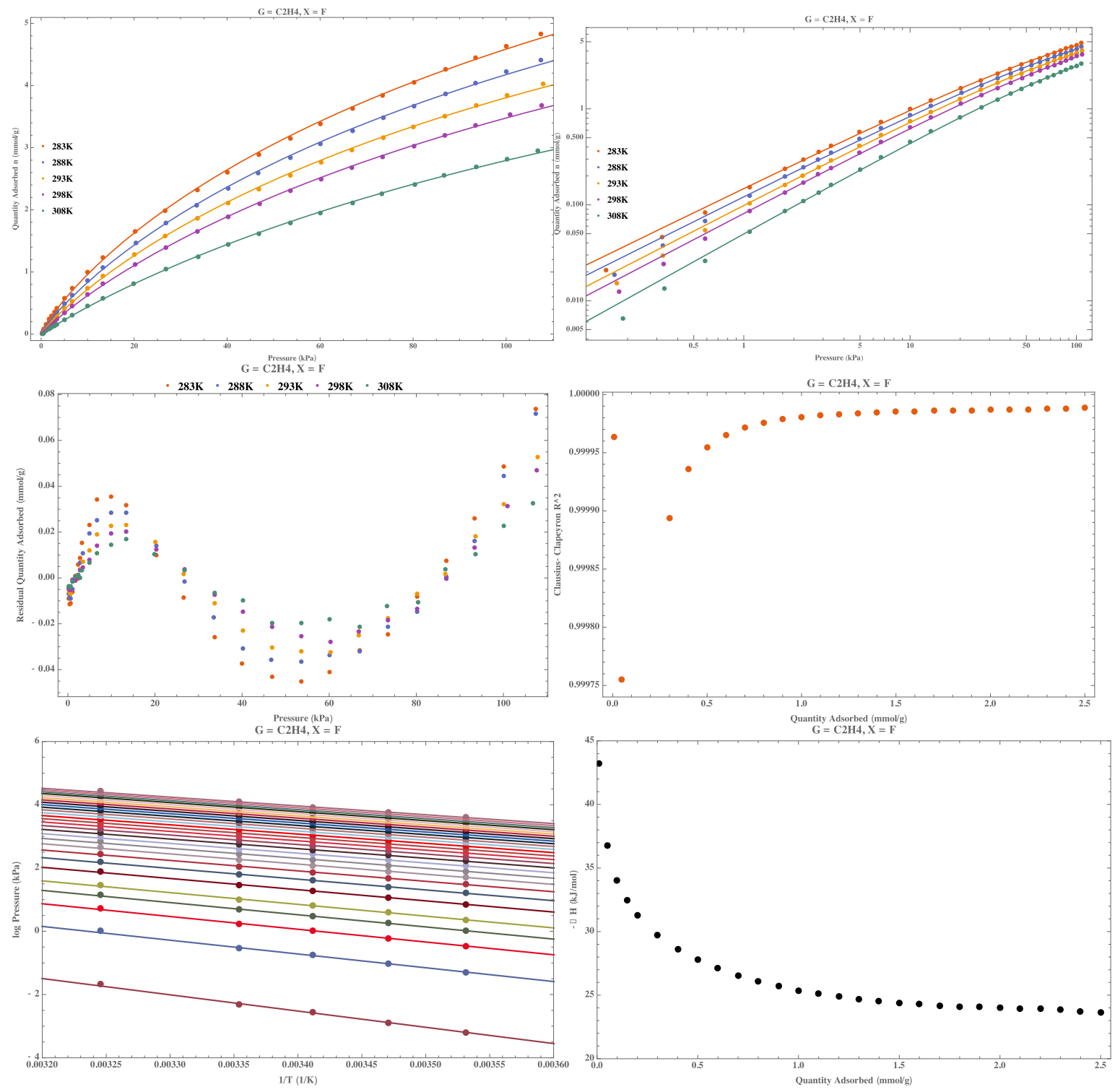

$\mathrm{G}=\mathrm{C} 2 \mathrm{H} 4, \mathrm{X}=\mathrm{Cl}$

\begin{tabular}{|l|l|l|l|l|l|}
\hline & $283 \mathrm{~K}$ & $288 \mathrm{~K}$ & $293 \mathrm{~K}$ & $298 \mathrm{~K}$ & $308 \mathrm{~K}$ \\
\hline $\mathrm{U}$ & 21.7058 & 21.6453 & 22.2085 & 24.016 & 24.5239 \\
\hline $\mathrm{S}$ & 0.943901 & 0.947855 & 0.949111 & 0.950006 & 0.953837 \\
\hline $\mathrm{a}$ & 0.00333629 & 0.00289391 & 0.00244852 & 0.00192215 & 0.00139425 \\
\hline $\mathrm{R}^{2}$ & 0.999995 & 0.999991 & 0.999993 & 0.999995 & 0.999989 \\
\hline
\end{tabular}



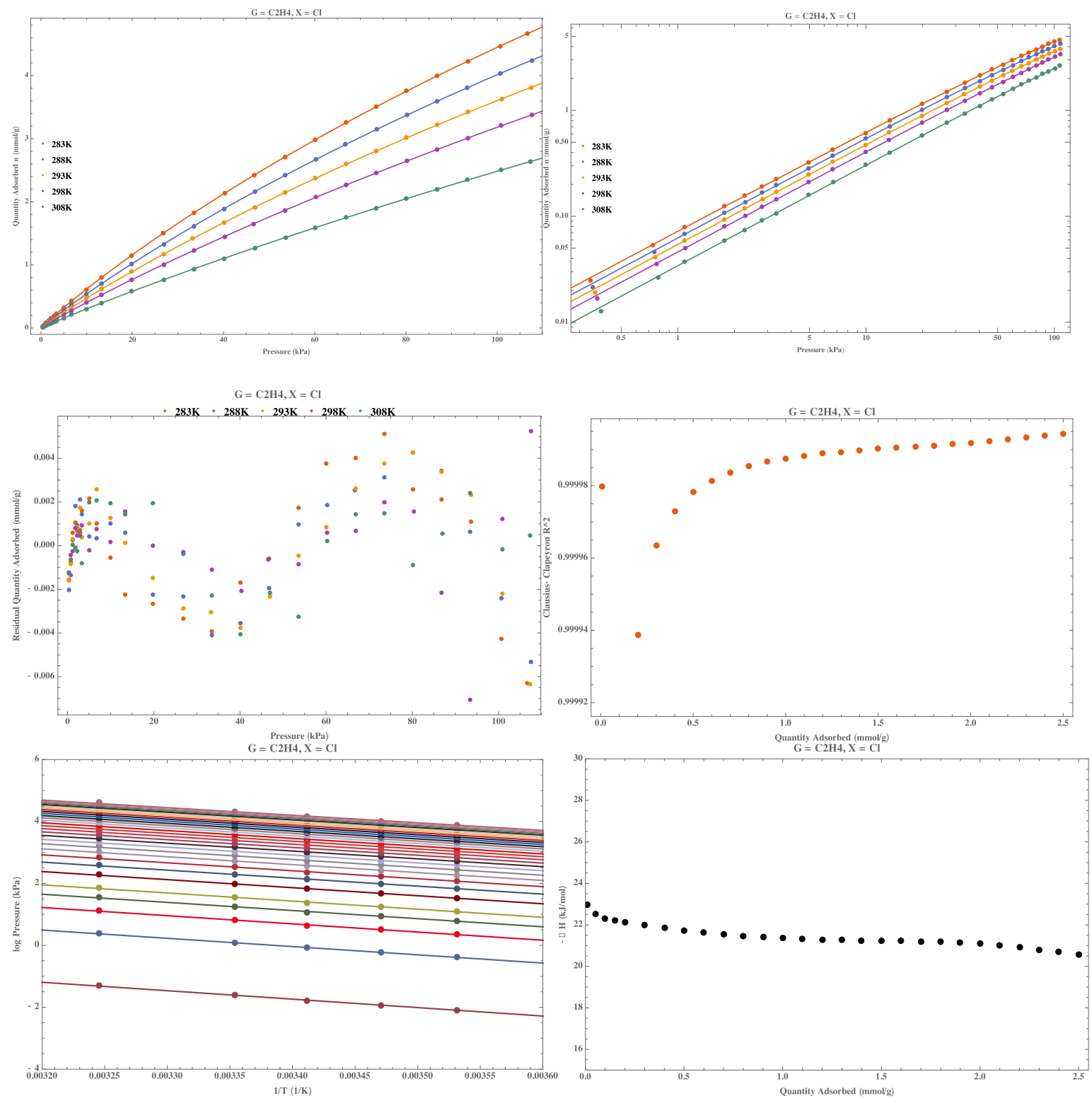

$\mathrm{G}=\mathrm{C} 2 \mathrm{H} 4, \mathrm{X}=\mathrm{Br}$

\begin{tabular}{|l|l|l|l|l|l|}
\hline & $283 \mathrm{~K}$ & $288 \mathrm{~K}$ & $293 \mathrm{~K}$ & $298 \mathrm{~K}$ & $308 \mathrm{~K}$ \\
\hline $\mathrm{U}$ & 49.8223 & 152.321 & 149.263 & 429.556 & 94.3708 \\
\hline $\mathrm{S}$ & 0.923228 & 0.908901 & 0.911411 & 0.906088 & 0.919935 \\
\hline $\mathrm{a}$ & 0.00108089 & 0.000321164 & 0.000286022 & 0.0000892223 & 0.000295874 \\
\hline $\mathrm{R}^{2}$ & 0.999971 & 0.999972 & 0.999985 & 0.999966 & 0.999929 \\
\hline
\end{tabular}



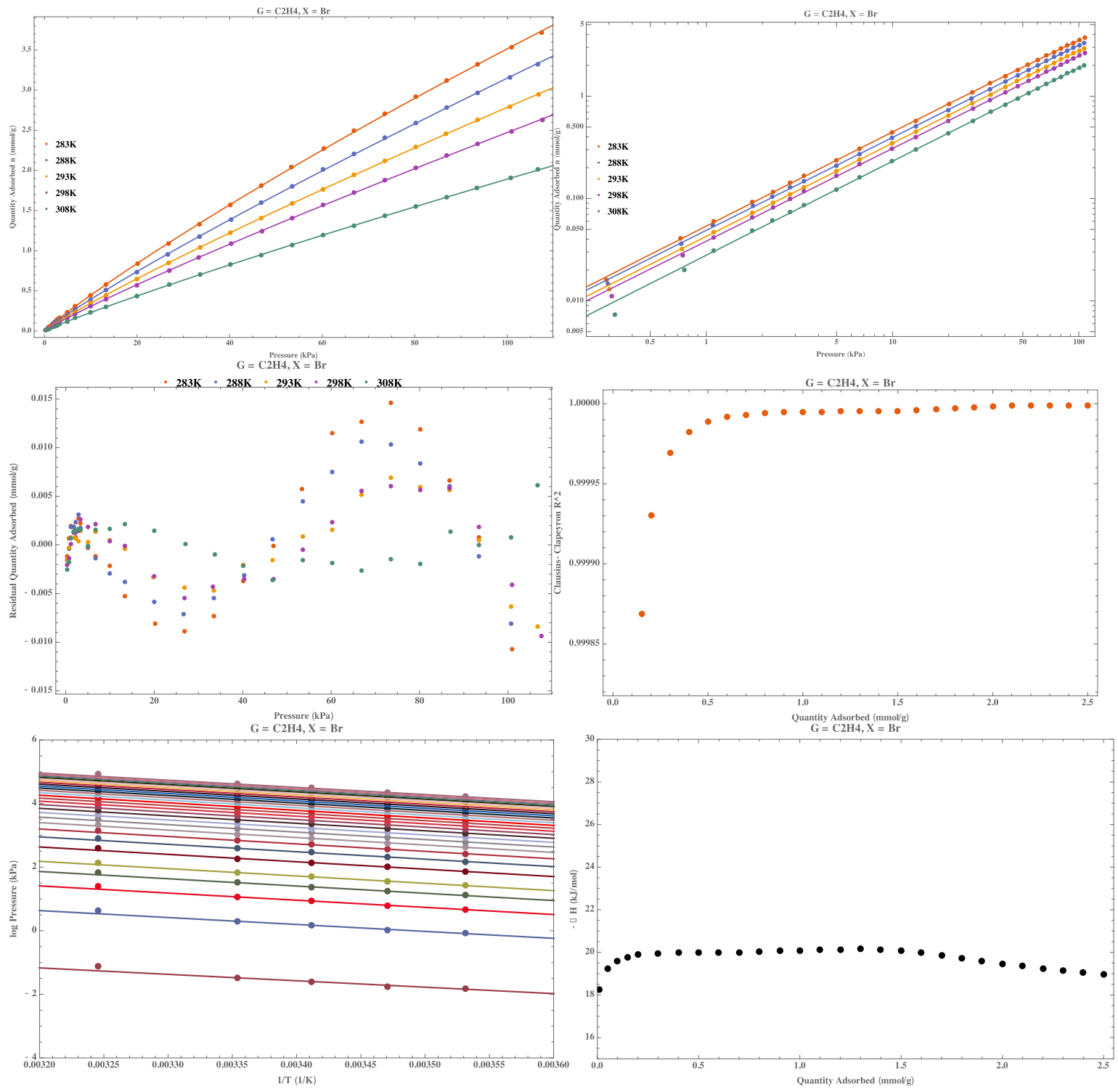


\section{Section 6.5. Dual-Site Langmuir Model for Isosteric Enthalpy of Adsorption}

$n=\frac{U_{1} a_{1} p}{1+a_{1} p}+\frac{U_{2} a_{2} p}{1+a_{2} p}$

$\mathrm{n}=$ quantity adsorbed $(\mathrm{mmol} / \mathrm{g})$

$\mathrm{p}=$ pressure $(\mathrm{kPa})$

$\mathrm{U}_{1}=$ maximum adsorption capacity site 1

$a_{1}=$ Langmuir constant for site 1

$\mathrm{U}_{2}=$ maximum adsorption capacity site 2

a2 = Langmuir constant for site 2

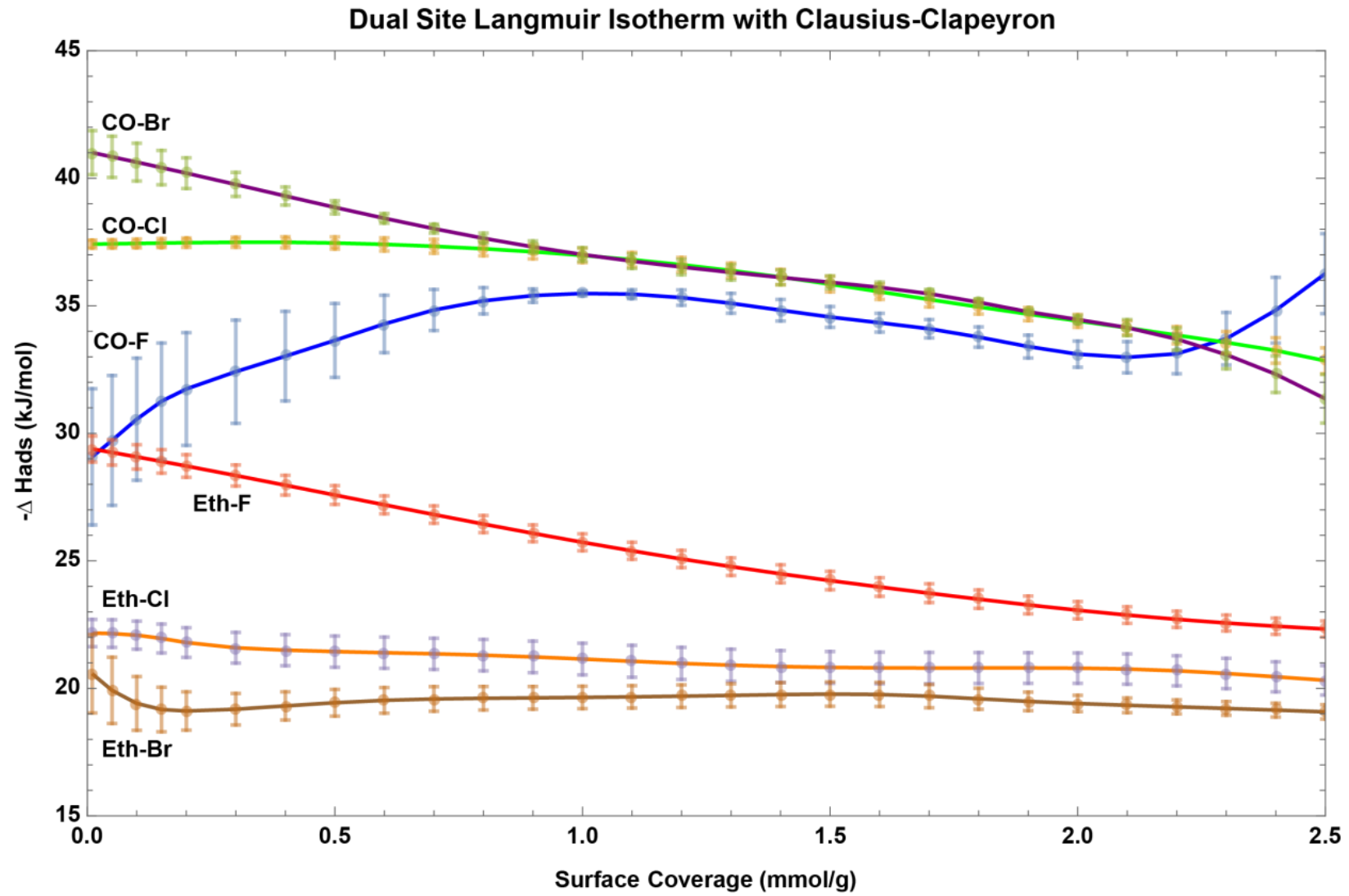

Summary of all DSL/CC calculations. Error bars show one standard deviation.

$\mathrm{G}=\mathrm{CO}, \mathrm{X}=\mathrm{F}$

\begin{tabular}{|l|l|l|l|l|l|}
\hline & $283 \mathrm{~K}$ & $288 \mathrm{~K}$ & $293 \mathrm{~K}$ & $298 \mathrm{~K}$ & $308 \mathrm{~K}$ \\
\hline $\mathrm{U}_{1}$ & 1.76323 & 2.7173 & 2.78848 & 2.84011 & 2.83802 \\
\hline $\mathrm{a}_{1}$ & 0.0935967 & 0.0127078 & 0.0104615 & 0.00894128 & 0.0130336 \\
\hline $\mathrm{U}_{2}$ & 3.12837 & 1.05535 & 0.999907 & 0.923821 & 0.187816 \\
\hline $\mathrm{a}_{2}$ & 0.00584444 & 0.118544 & 0.0937149 & 0.0746356 & 0.147748 \\
\hline
\end{tabular}




\begin{tabular}{|l|l|l|l|l|l|}
\hline $\mathrm{R}^{2}$ & 0.999826 & 0.999814 & 0.999884 & 0.999718 & 0.999587 \\
\hline
\end{tabular}
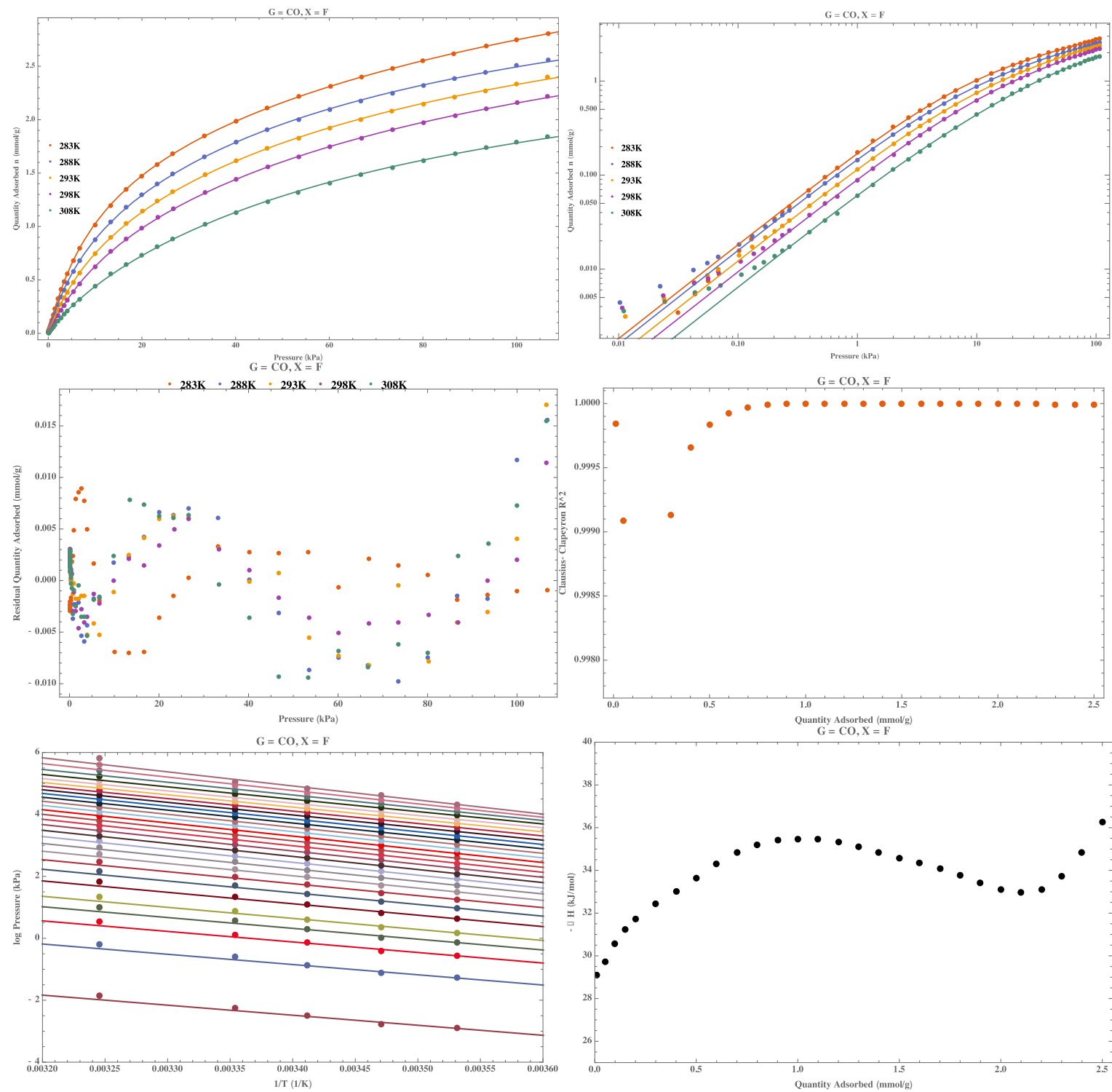

$\mathrm{G}=\mathrm{CO}, \mathrm{X}=\mathrm{Cl}$

\begin{tabular}{|l|l|l|l|l|l|}
\hline & $283 \mathrm{~K}$ & $288 \mathrm{~K}$ & $293 \mathrm{~K}$ & $298 \mathrm{~K}$ & $308 \mathrm{~K}$ \\
\hline $\mathrm{U}_{1}$ & 2.79425 & 1.45672 & 1.44297 & 2.9624 & 3.11188 \\
\hline $\mathrm{a}_{1}$ & 0.016538 & 0.121102 & 0.0931956 & 0.0104458 & 0.00687372 \\
\hline $\mathrm{U}_{2}$ & 1.52 & 2.83829 & 2.91287 & 1.20834 & 1.13045 \\
\hline $\mathrm{a}_{2}$ & 0.157121 & 0.0137755 & 0.0107251 & 0.0793118 & 0.0506936 \\
\hline $\mathrm{R}^{2}$ & 0.999985 & 0.999988 & 0.999993 & 0.999963 & 0.999991 \\
\hline
\end{tabular}



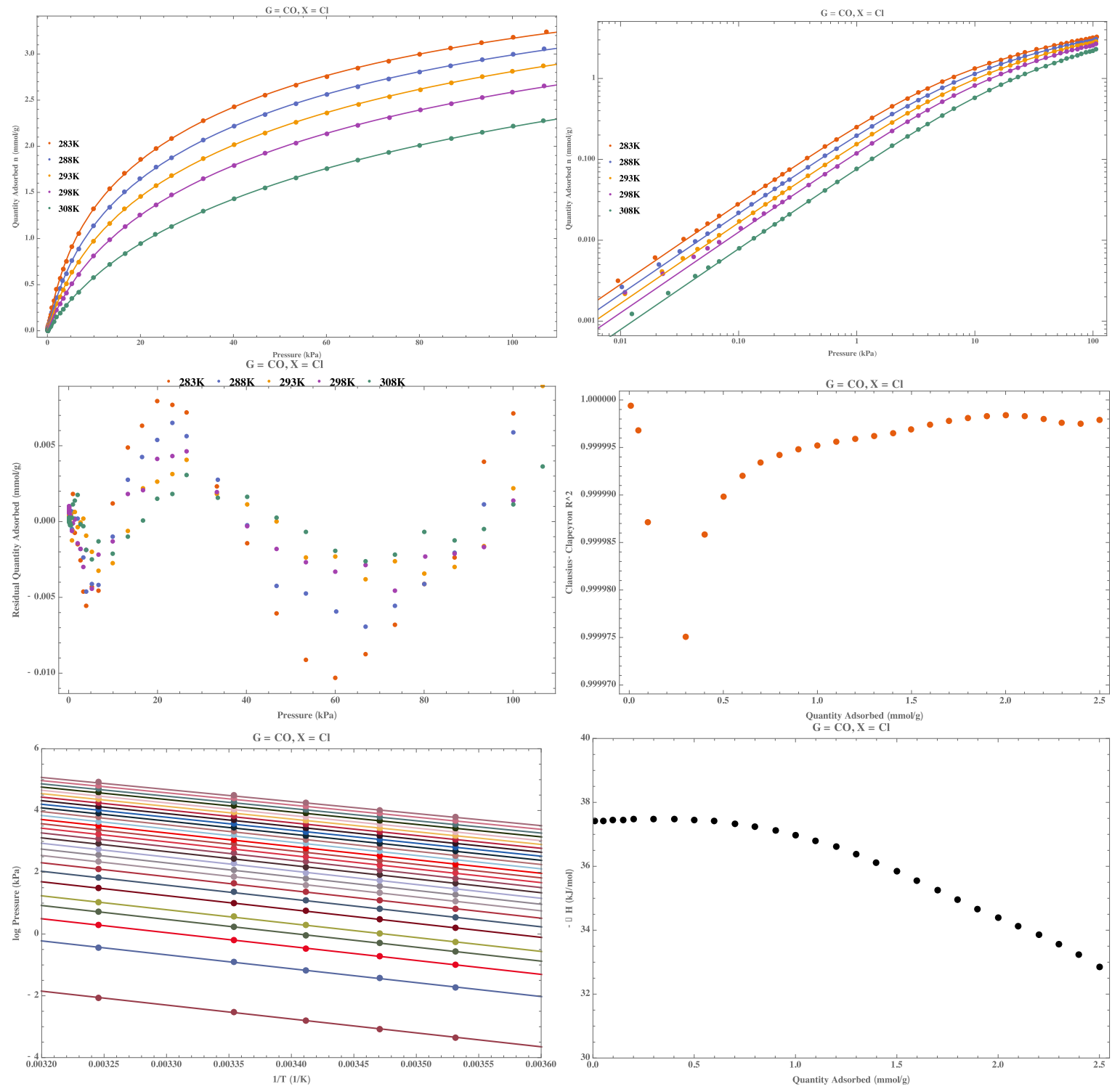

$\mathrm{G}=\mathrm{CO}, \mathrm{X}=\mathrm{Br}$

\begin{tabular}{|l|l|l|l|l|l|}
\hline & $283 \mathrm{~K}$ & $288 \mathrm{~K}$ & $293 \mathrm{~K}$ & $298 \mathrm{~K}$ & $308 \mathrm{~K}$ \\
\hline $\mathrm{U}_{1}$ & 2.46217 & 2.56768 & 2.418 & 2.44229 & 2.89267 \\
\hline $\mathrm{a}_{1}$ & 0.02135 & 0.0170786 & 0.00987337 & 0.0117264 & 0.00330234 \\
\hline $\mathrm{U}_{2}$ & 0.943152 & 0.868636 & 1.19003 & 0.866496 & 1.31304 \\
\hline $\mathrm{a}_{2}$ & 0.231705 & 0.182158 & 0.0998768 & 0.0096256 & 0.0423484 \\
\hline $\mathrm{R}^{2}$ & 0.999722 & 0.999698 & 0.999937 & 0.999844 & 0.999991 \\
\hline
\end{tabular}



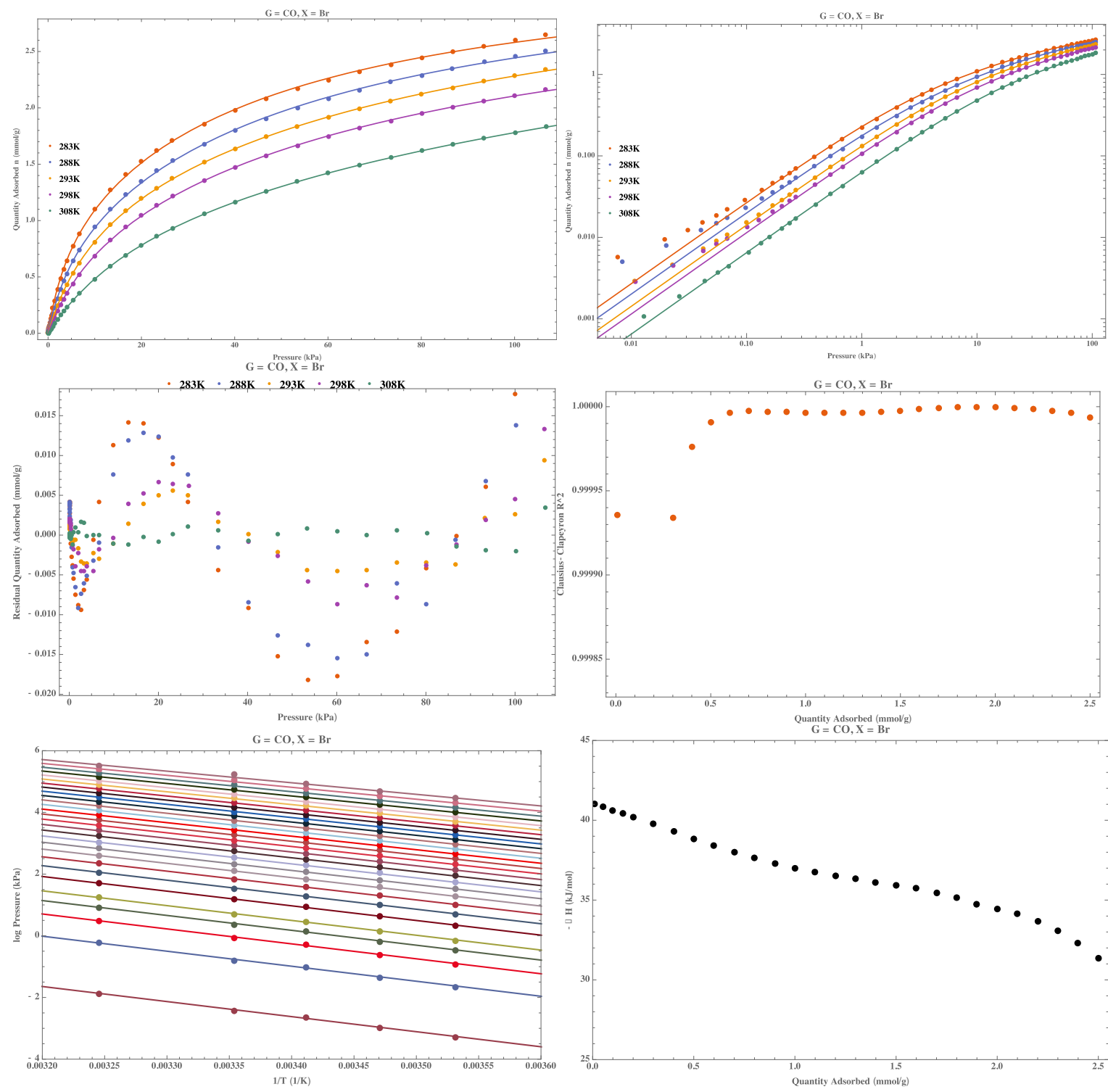

$\mathrm{G}=\mathrm{C}_{2} \mathrm{H}_{4}, \mathrm{X}=\mathrm{F}$

\begin{tabular}{|l|l|l|l|l|l|}
\hline & $283 \mathrm{~K}$ & $288 \mathrm{~K}$ & $293 \mathrm{~K}$ & $298 \mathrm{~K}$ & $308 \mathrm{~K}$ \\
\hline $\mathrm{U}_{1}$ & 13.1321 & 1.08773 & 1.05136 & 1.02448 & 1028.92 \\
\hline $\mathrm{a}_{1}$ & 0.00384017 & 0.0689303 & 0.0540611 & 0.044034 & 0.0000145715 \\
\hline $\mathrm{U}_{2}$ & 1.10213 & 13.0558 & 12.1511 & 12.9691 & 2.14487 \\
\hline $\mathrm{a}_{2}$ & 0.0872416 & 0.00333717 & 0.00320464 & 0.00259274 & 0.00259274 \\
\hline $\mathrm{R}^{2}$ & 0.999997 & 0.999996 & 0.999992 & 0.999988 & 0.99992 \\
\hline
\end{tabular}



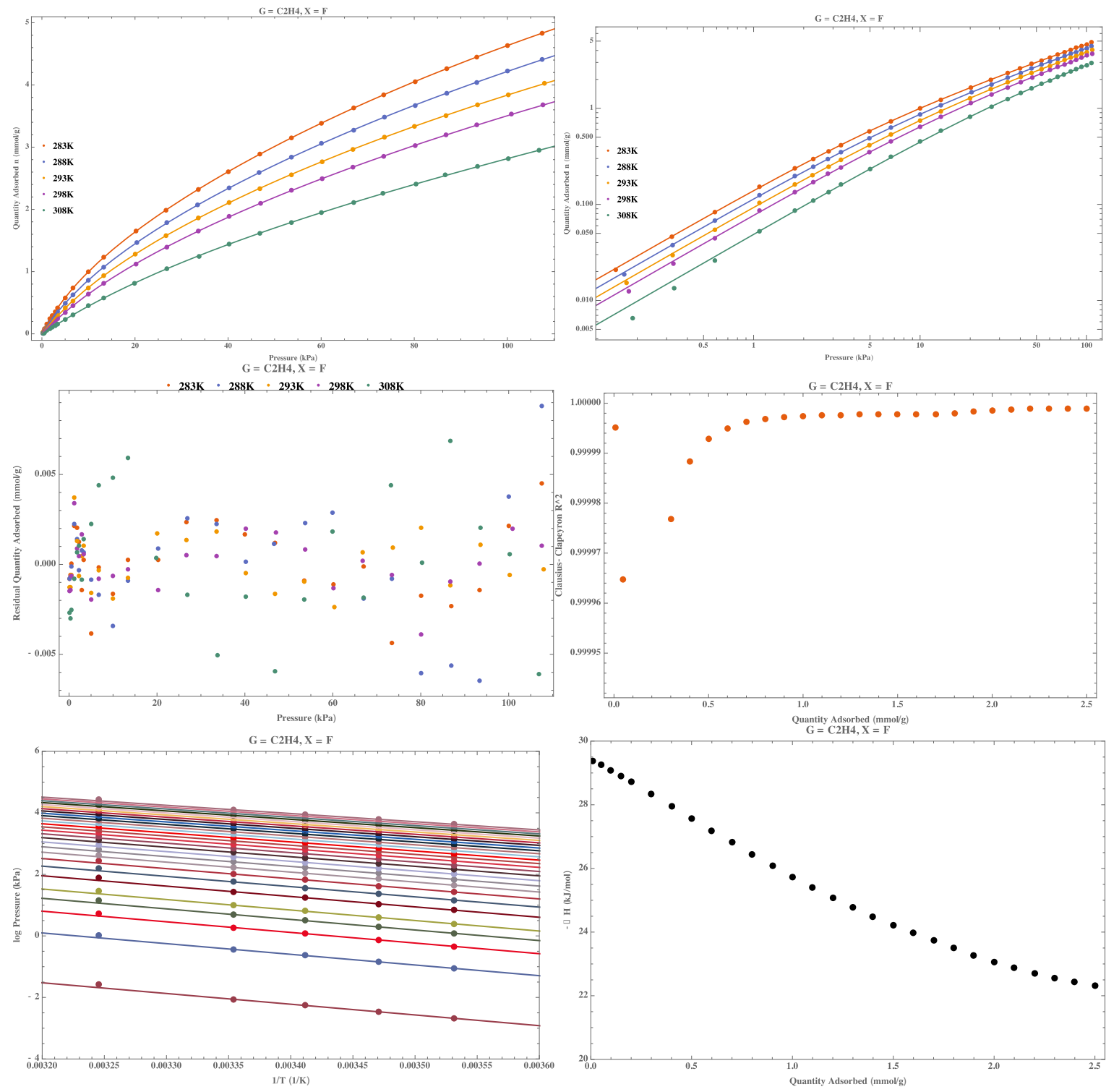

$\mathrm{G}=\mathrm{C}_{2} \mathrm{H}_{4}, \mathrm{X}=\mathrm{Cl}$

\begin{tabular}{|l|l|l|l|l|l|}
\hline & $283 \mathrm{~K}$ & $288 \mathrm{~K}$ & $293 \mathrm{~K}$ & $298 \mathrm{~K}$ & $308 \mathrm{~K}$ \\
\hline $\mathrm{U}_{1}$ & 0.0889428 & 17.9032 & 0.0774436 & 18.8628 & 0.0706771 \\
\hline $\mathrm{a}_{1}$ & 0.19005 & 0.00281494 & 0.146105 & 0.00198009 & 0.0914834 \\
\hline $\mathrm{U}_{2}$ & 17.7817 & 0.0848068 & 18.0982 & 0.0678695 & 20.0265 \\
\hline $\mathrm{a}_{2}$ & 0.00324847 & 0.158996 & 0.00242382 & 0.136973 & 0.00137576 \\
\hline $\mathrm{R}^{2}$ & 0.999999 & 0.999997 & 0.999998 & 0.999997 & 0.999997 \\
\hline
\end{tabular}



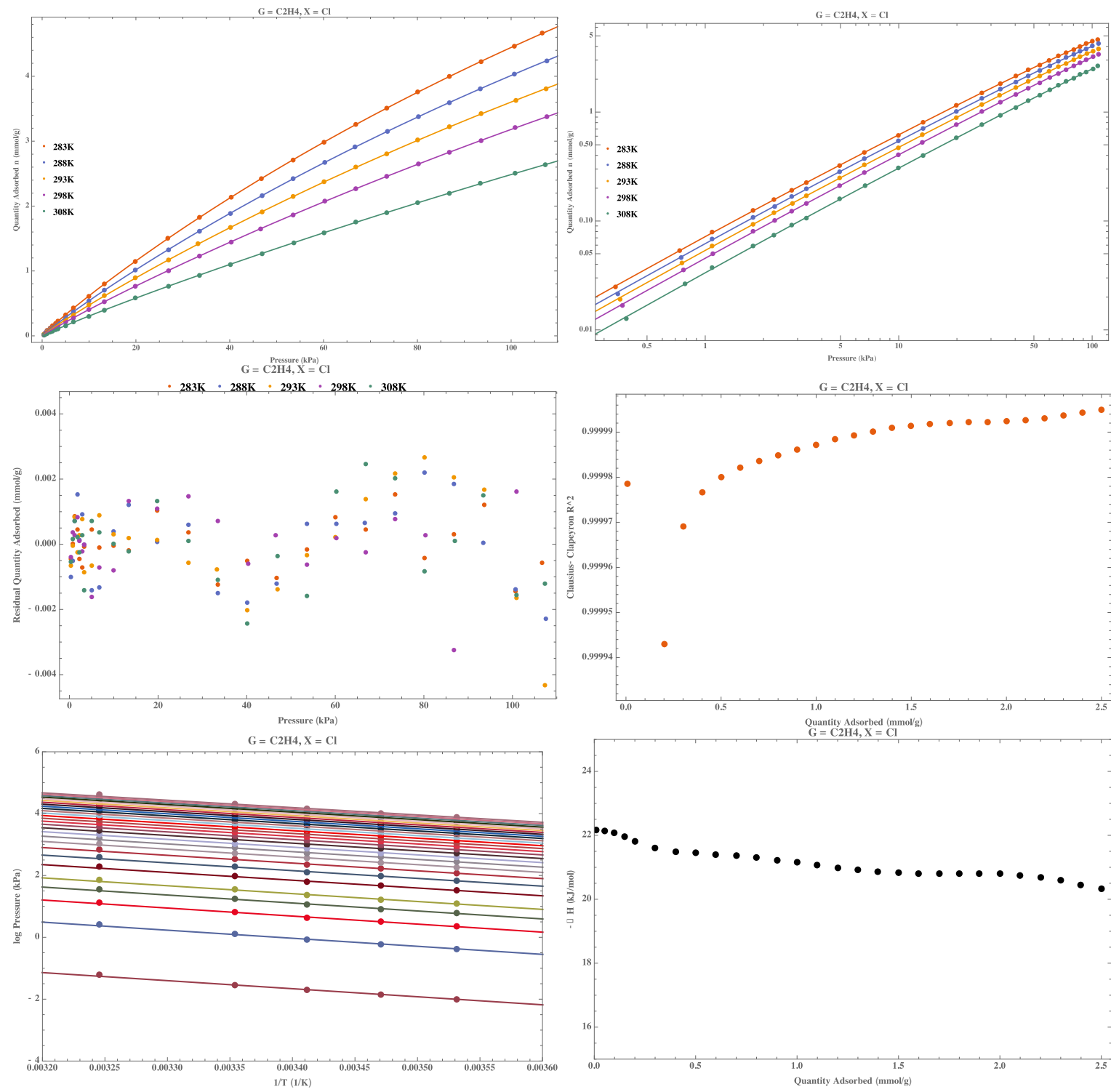

$\mathrm{G}=\mathrm{C}_{2} \mathrm{H}_{4}, \mathrm{X}=\mathrm{Br}$

\begin{tabular}{|l|l|l|l|l|l|}
\hline & $283 \mathrm{~K}$ & $288 \mathrm{~K}$ & $293 \mathrm{~K}$ & $298 \mathrm{~K}$ & $308 \mathrm{~K}$ \\
\hline $\mathrm{U}_{1}$ & 22.9129 & 26.4185 & 26.6506 & 27.8703 & 30.7648 \\
\hline $\mathrm{a}_{1}$ & 0.00177518 & 0.00132047 & 0.00113493 & 0.000941937 & 0.000627983 \\
\hline $\mathrm{U}_{2}$ & 0.0565859 & 0.0647446 & 0.0702615 & 0.076716 & 0.0843938 \\
\hline $\mathrm{a}_{2}$ & 0.33456 & 0.292574 & 0.210294 & 0.177744 & 0.10093 \\
\hline $\mathrm{R}^{2}$ & 0.999993 & 0.999995 & 0.999996 & 0.999993 & 0.999967 \\
\hline
\end{tabular}



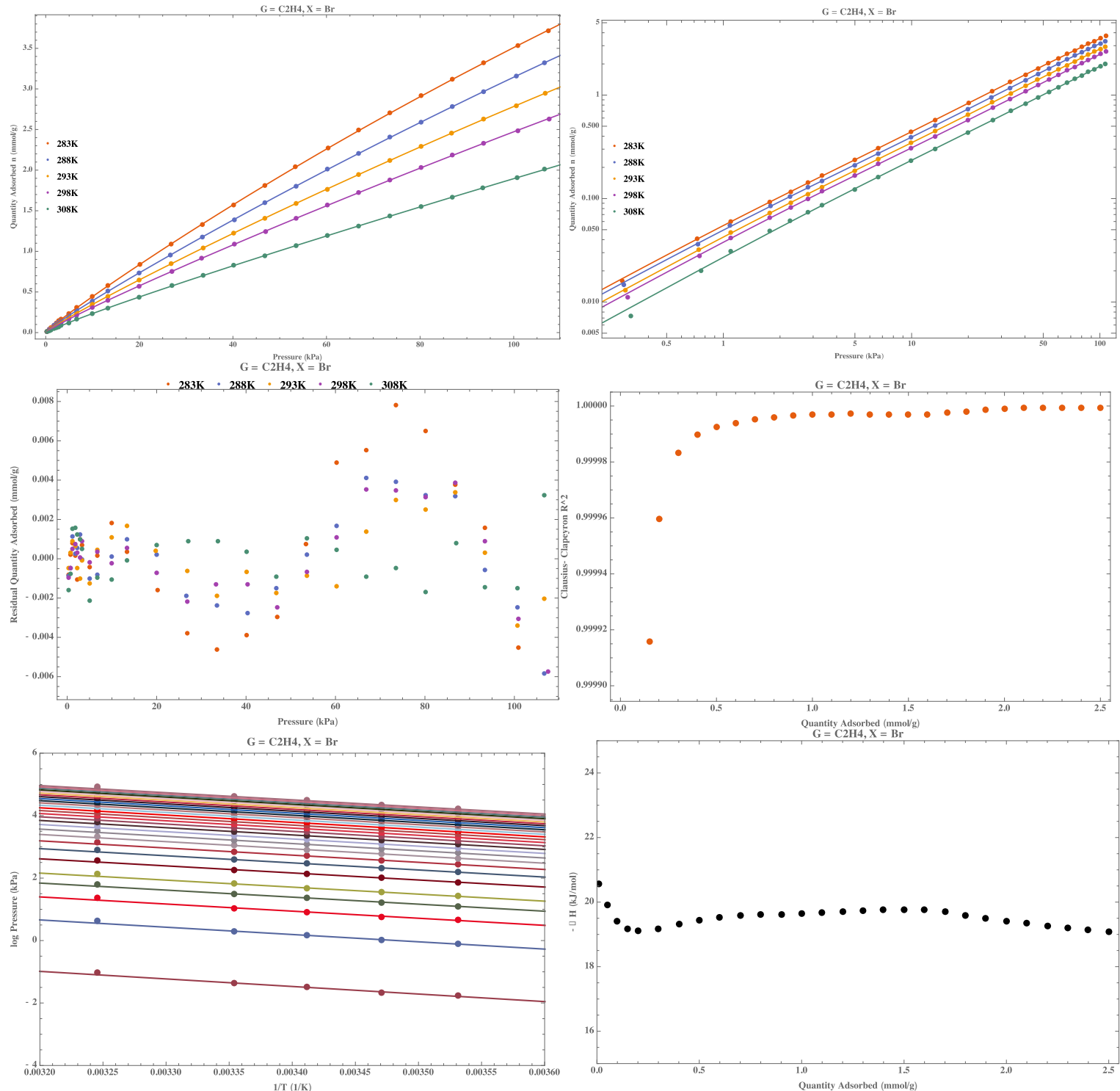


\section{S6. Notebook for calculation of}

isosteric enthalpy of adsorption from variable temperature isotherms

Written using Mathematica 11

Import data from Excel file s=Import[".../NiCIBTDD_CO_VT_data.xlsx"];

Parse the Excel file, loading each pressure and quantity adsorbed into an array.

In this example, the data has already been converted into $\mathrm{kPa}$ and $\mathrm{mmol} / \mathrm{g}$ in the Excel file.

Parsing an Excel file proceeds at the following $s[[a]][[A l l, b]][[c ; ; d]]$ with 'a' is the Excel page, ' $b$ ' is the column number, ' $c$ ' is the first row, and ' $d$ ' is the last row. Counting starts at 1.

p283=s[[2]][[All,1]][[4;;44]]; $\left({ }^{*}\right.$ Pressure at $283 \mathrm{~K}$ in units of $\left.\mathrm{kPa}{ }^{*}\right)$ q283=s[[2]][[All,3]][[4;;44]]; ( ${ }^{*}$ Quantity adsorbed at $283 \mathrm{~K}$ in units of $\mathrm{mmol} / \mathrm{g}$ *) p288=s[[2]][[All,5]][[4;;44]];

q288=s[[2]][[All,7]][[4;;44]];

p293=s[[2]][AII,9]][[4;;44]];

q293=s[[2]][[All,11]][[4;;44]];

p298=s[[2]][[All, 13]][[4;;43]];

q298=s[[2]][[All,15]][[4;;43]];

p308=s[[2]][[All, 17]][[4;;43]];

q308=s[[2]][[All,19]][[4;;43]];

Make an array of 'quantity adsorbed' points to which the isosteric enthalpy will be calculated in the final result $(\mathrm{mmol} / \mathrm{g})$.

Remember to not extrapolate outside of the experimentally measured quantities. In this example, the minimum experimentally measured quantity adsorbed is around 0.001 and maximum is 3 .

plist $=\{0.01,0.05,0.1,0.15,0.2,0.3,0.4,0.5,0.6,0.7,0.8,0.9,1.0,1.1,1.2,1.3,1.4,1.5,1.6$ $, 1.7,1.8,1.9,2.0,2.1,2.2,2.3,2.4,2.5\}$; 
Fit the data to a model. In this case, the Unilan function. The data is weighted as $1 /$ 'quantity adsorbed' as the fit at small pressures will dictate the accuracy of the zero-pressure isosteric enthalpy of adsorption.

udnlm283=NonlinearModelFit[Transpose@\{p283,q283\},\{u/(2*ss)* Log $\left[\left(1+1 / a^{*} p^{*} E\right.\right.$ $\left.\left.x p[s s]) /\left(1+1 / a^{*} p^{*} \operatorname{Exp}[-s s]\right)\right]\right\},\{\{u, 5\},\{s s, 2\},\{a, 10\}\}, p$, Maxlterations- $>50000$, Weights$>1 / q 283]$;

udnlm288=NonlinearModelFit[Transpose@ @p288,q288\},\{u/(2*ss)* $\log \left[\left(1+1 / \mathrm{a}^{*} \mathrm{p}^{\star} \mathrm{E}\right.\right.$ $\left.\left.x p[s s]) /\left(1+1 / a^{*} p^{*} \operatorname{Exp}[-s s]\right)\right]\right\},\{\{u, 5\},\{s s, 2\},\{a, 10\}\}, p, M a x l t e r a t i o n s->50000$, Weights$>1 / q 288]$;

udnlm293=NonlinearModelFit[Transpose@\{p293,q293\},\{u/(2*ss)*Log[(1+1/a* ${ }^{*} E$ $\left.\left.x p[s s]) /\left(1+1 / a^{*} p^{\star} \operatorname{Exp}[-s s]\right)\right]\right\},\{\{u, 5\},\{s s, 2\},\{a, 10\}\}, p$, Maxlterations- $>50000$, Weights$>1 / q 293]$

udnlm298=NonlinearModelFit[Transpose@\{p298,q298\},\{u/(2*ss)* Log $\left[\left(1+1 / a^{*} p^{*} E\right.\right.$ $\left.\left.x p[s s]) /\left(1+1 / a^{*} p^{\star} \operatorname{Exp}[-s s]\right)\right]\right\},\{\{u, 5\},\{s s, 2\},\{a, 10\}\}, p$, Maxlterations- $>50000$, Weights$>1 / q 298]$

udnIm308=NonlinearModelFit[Transpose@\{p308,q308\},\{u/(2*ss)*Log[(1+1/a* ${ }^{*} E$ $\left.\left.x p[s s]) /\left(1+1 / a^{*} p^{*} \operatorname{Exp}[-s s]\right)\right]\right\},\{\{u, 25\},\{s s, 2\},\{a, 100\}\}, p$, Maxlterations-

$>50000$, Weights->1/q308];

Inspect the results from the fit. The R2 value should be above 0.999

udnlm283["BestFitParameters"]

udnlm288["BestFitParameters"]

udnlm293["BestFitParameters"]

udnlm298["BestFitParameters"]

udnlm308["BestFitParameters"]

udnlm283["RSquared"]

udnlm288["RSquared"]

udnlm293["RSquared"]

udnlm298["RSquared"]

udnlm308["RSquared"]

udnIm283["ParameterTable"]

udnlm288["ParameterTable"]

udnIm293["ParameterTable"]

udnlm298["ParameterTable"]

udnlm308["ParameterTable"]

$\{u->5.0222, s s->2.58975, a->44.5242\}$

$\{u->5.19379$, ss->2.62905,a->62.6492\} 


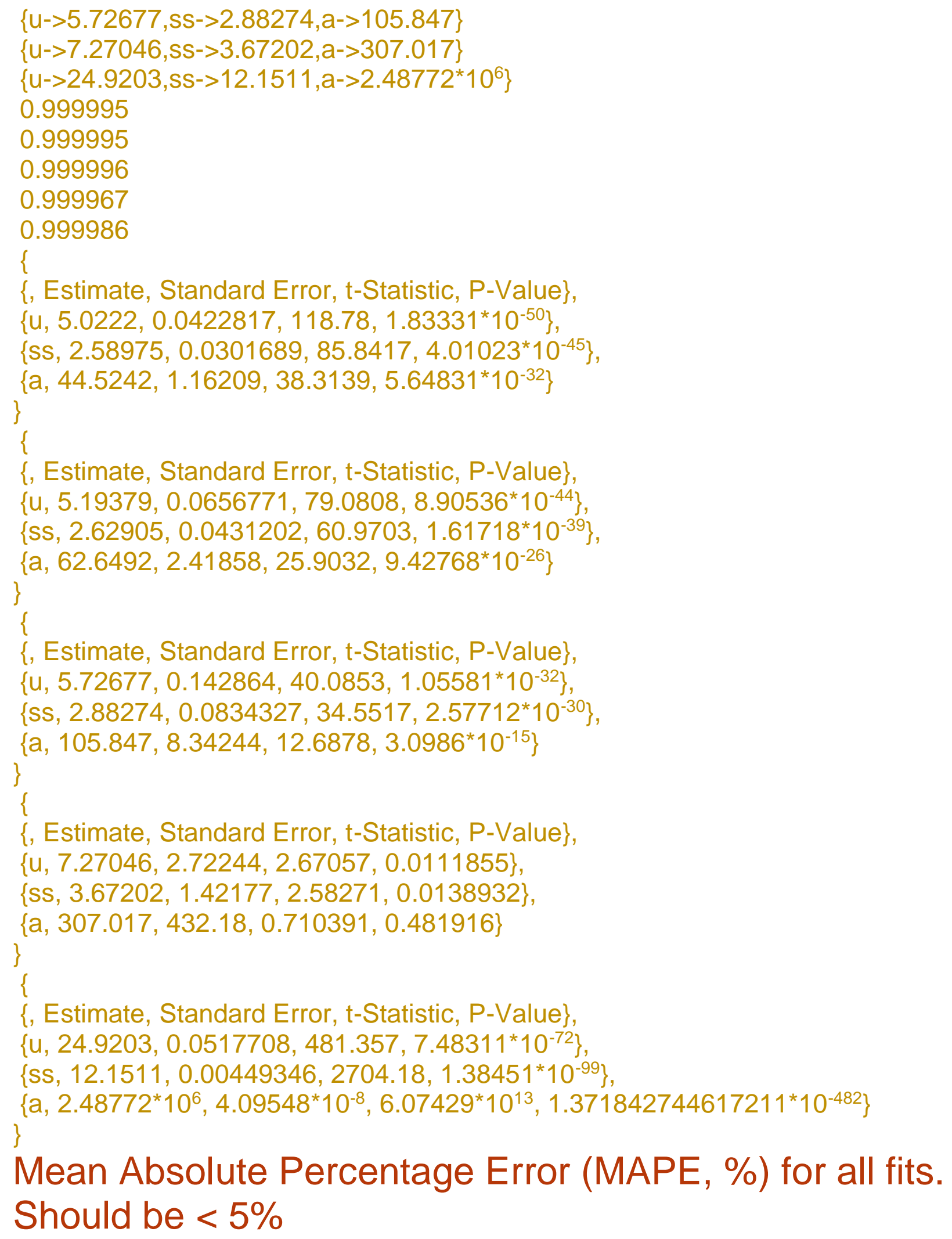


For[i=1, $i<$ Length[p283]+1, $\mathrm{i}++$, mape283+=Abs[(q283[[i]]udnlm283[p283[[i]]])/q283[[i]]]]

mape283=mape283/Length[p283] ${ }^{\star} 100$;

mape288=0;

For $[\mathrm{i}=1, \mathrm{i}<$ Length[p288]+1, $\mathrm{i}++$, mape288+=Abs[(q288[[i]]udnlm288[p288[[i]]])/q288[[i]]]]

mape288=mape288/Length[p288] ${ }^{\star} 100$;

mape293=0;

For[i=1,i<Length[p293]+1, i++, mape293+=Abs[(q293[[i]]-

udnlm293[p293[[i]]])/q293[[i]]]]

mape293=mape293/Length[p293] ${ }^{\star} 100$;

mape298=0;

For $[i=1, i<L$ ength[p298]+1, $\mathrm{i}++$, mape298+=Abs[(q298[[i]]udnlm298[p298[[i]]])/q298[[i]]]]

mape298=mape298/Length[p298] ${ }^{\star} 100$;

mape308=0;

For $[\mathrm{i}=1, \mathrm{i}<$ Length[p308]+1, $\mathrm{i}++$, mape308+=Abs[(q308[[i]]udnlm308[p308[[i]]])/q308[[i]]]]

mape308=mape308/Length[p308] ${ }^{\star} 100$;

(mape283+mape288+mape293+mape298+mape308)/5

1.61171

Linear-linear plot of data with fits

Show[ListPlot[\{Transpose@\{p283,q283\},Transpose@\{p288,q288\},Transpose@\{ p293,q293\},Transpose@\{p298,q298\},Transpose@\{p308,q308\}\},PlotTheme>"Scientific",FrameLabel->\{"Pressure (kPa)","Quantity Adsorbed (mmol/g)"\},PlotLabel->"G = CO, X = Cl",PlotLegends>Placed[\{"283K","288K","293K","298K","308K"\},\{Left,Center\}]],Plot[\{udnlm283[x], udnlm288[x],udnlm293[x],udnlm298[x],udnlm308[x]\},\{x,0.00001,150\},PlotTheme>"Scientific"],ImageSize->Full,FrameStyle->Black] 


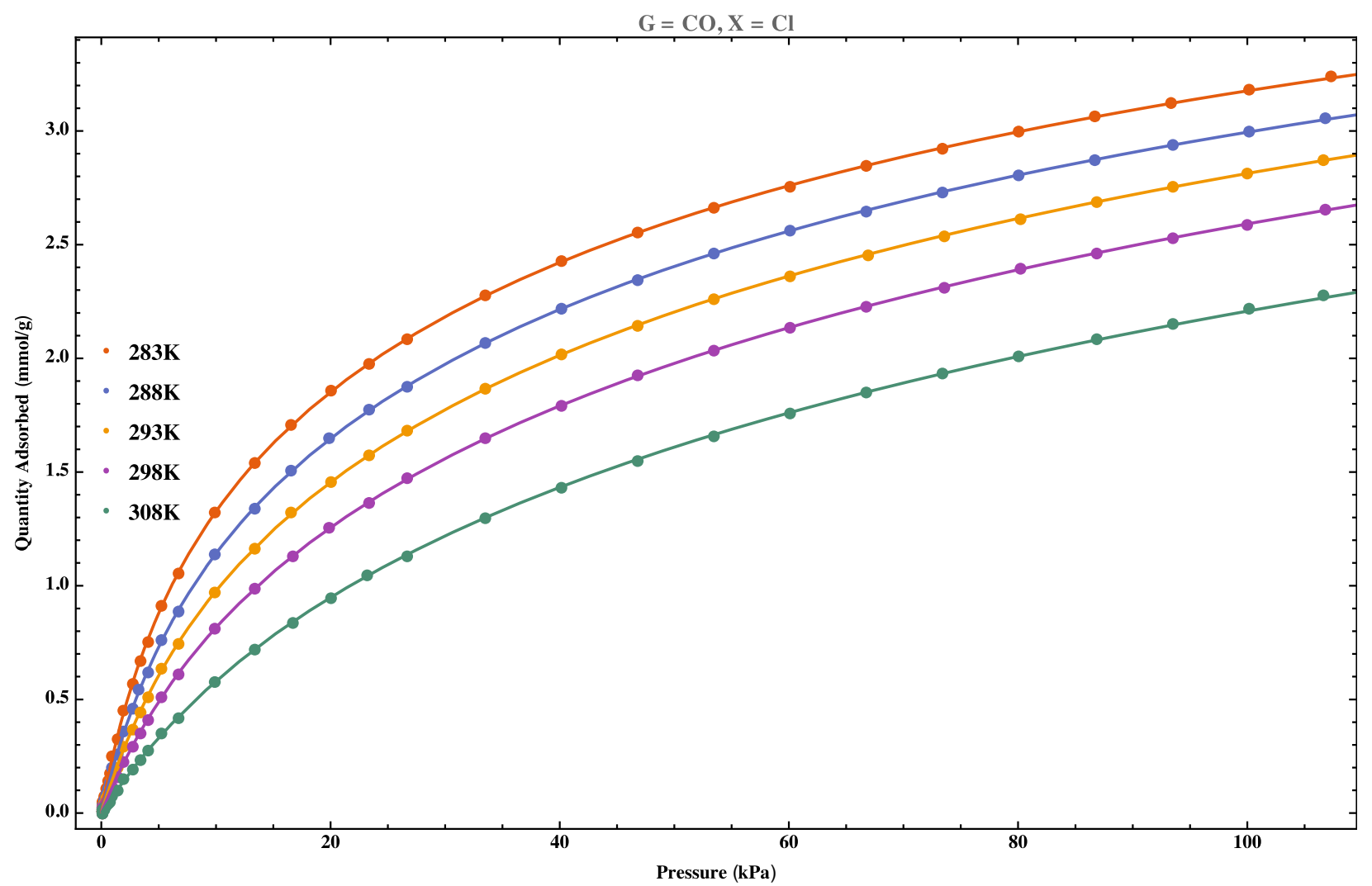

Log-log plot of data and fits. The log-log plot is necessary to visualize the fit at low pressures.

Show[ListLogLogPlot[\{Transpose@\{p283,q283\},Transpose@\{p288,q288\},Transp ose@\{p293,q293\},Transpose@\{p298,q298\},Transpose@\{p308,q308\}\},PlotThem e->"Scientific",FrameLabel->\{"Pressure (kPa)","Quantity Adsorbed (mmol/g)"\},PlotLabel->"G = CO, X = Cl",PlotLegends>Placed[\{"283K","288K","293K","298K","308K"\},\{Left,Center\}]],LogLogPlot[\{udnl m283[x],udnlm288[x],udnlm293[x],udnlm298[x],udnlm308[x]\},\{x,0.00001,100\},Plo tTheme->"Scientific"],ImageSize->Full,FrameStyle->Black] 


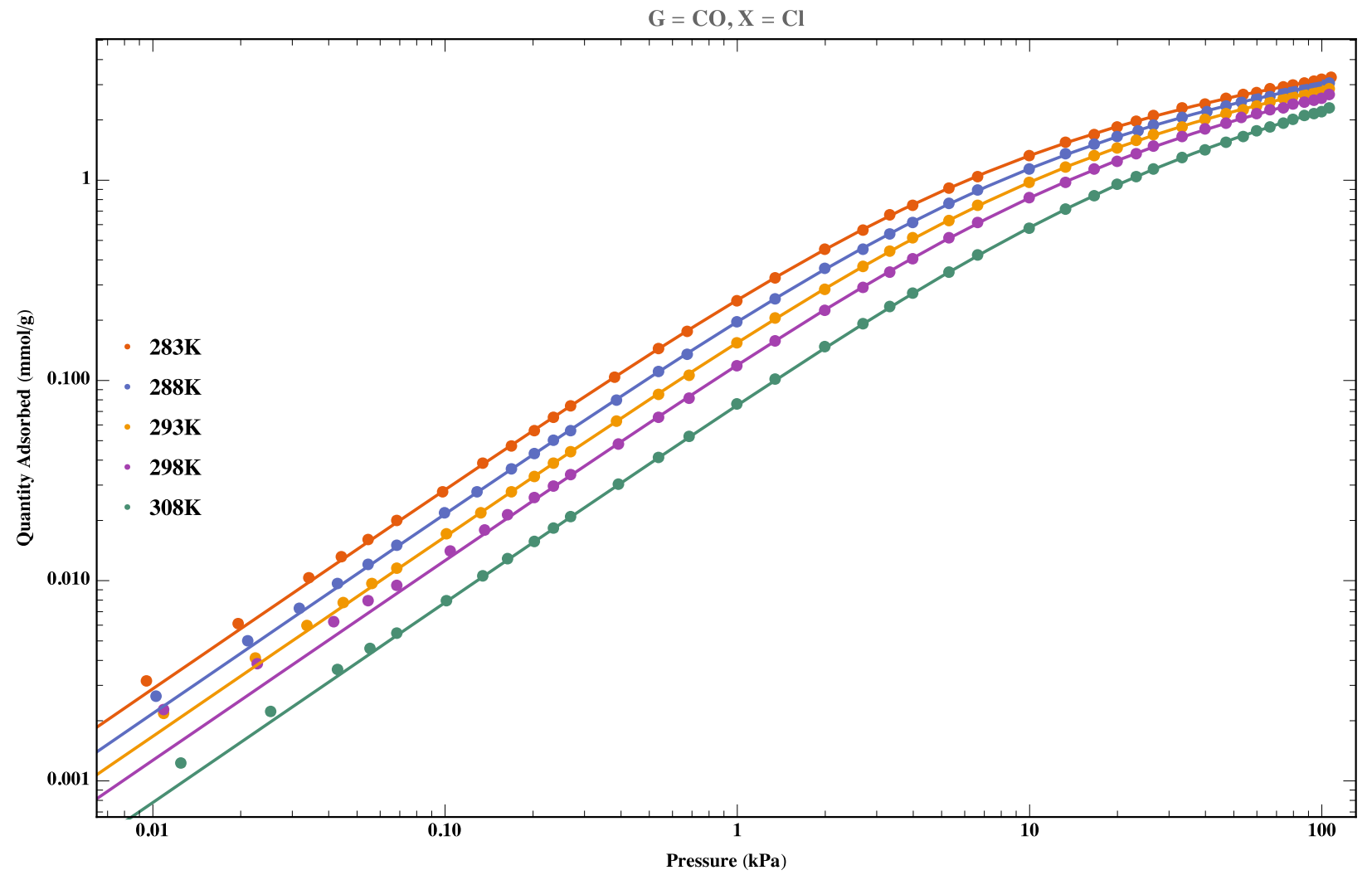

\section{Plot the residuals from the fit.}

ListPlot[\{Transpose@\{p283,udnlm283["FitResiduals"]\},Transpose@\{p288,udnlm 288["FitResiduals"]\},Transpose@\{p293,udnlm293["FitResiduals"]\},Transpose@\{ p298,udnlm298["FitResiduals"]\},Transpose@\{p308,udnlm308["FitResiduals"]\}\},PI otTheme->"Scientific",FrameLabel->\{"Pressure (kPa)","Residual Quantity Adsorbed (mmol/g)"\},PlotLabel->"G = CO, X = Cl",PlotLegends>Placed[\{"283K","288K","293K","298K","308K"\},\{Top,Center\}],ImageSize$>$ Large,PlotRange->\{\{0,110\},\{-0.01,0.01\}\},FrameStyle->Black] 


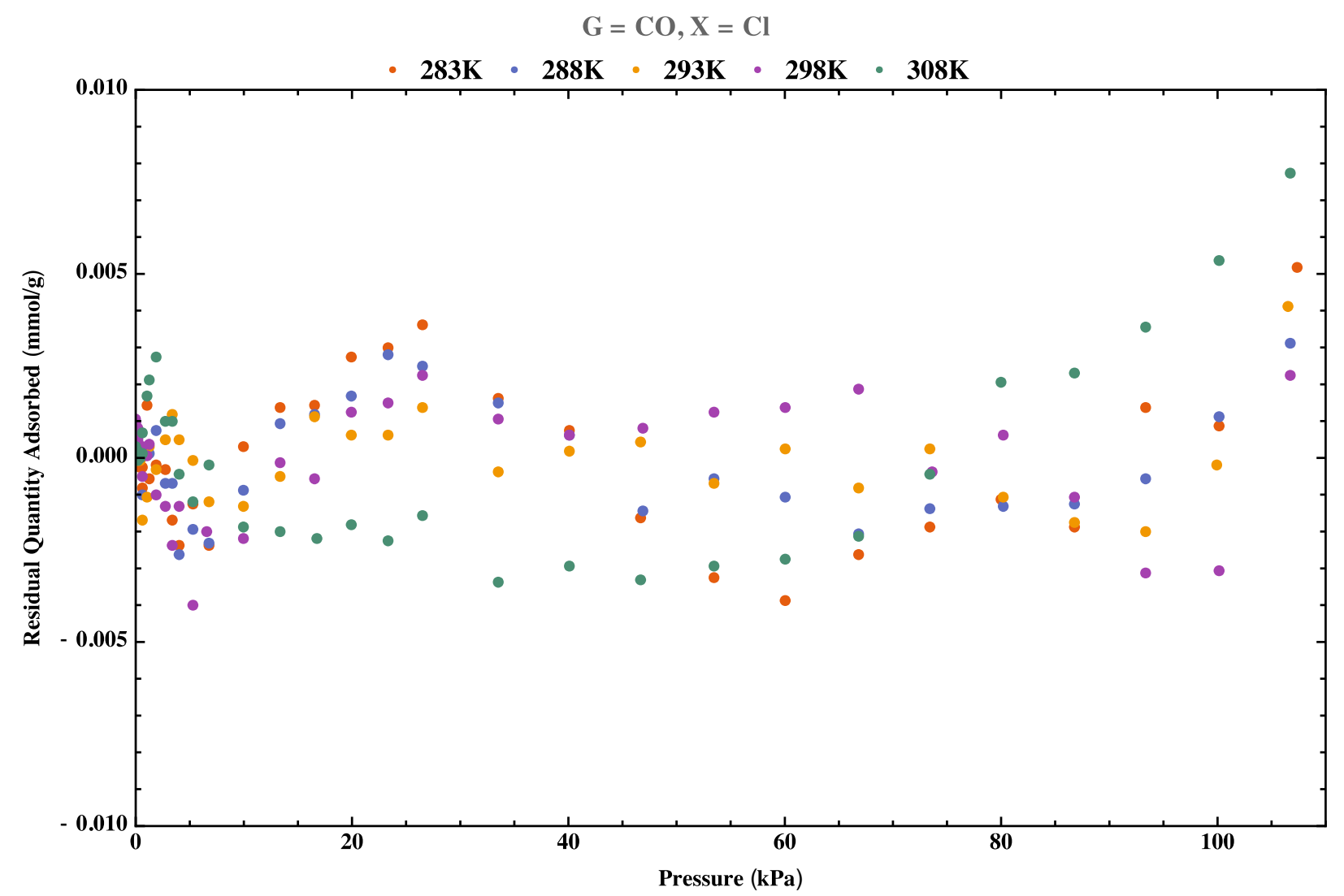

Calculate $P(Q)$ from $Q(P)$. Most isotherm models are derived for quantity adsorbed as a function of pressure, but as the isosteric enthalpy of adsorption is a function of quantity adsorbed, we will invert $Q(P)$. This is not strictly necessary, but allows the errors to be calculated easily. Note that not all isotherm models can be inverted analytically.

Simplify[Refine[Solve[n==u/(2*ss)* Log[(1+1/a* $\left.p^{*} \operatorname{Exp}[s s]\right) /\left(1+1 / a^{*} p^{*} \operatorname{Exp}[-\right.$ ss])],p],\{Element[ss, Reals],Element[n,Reals],Element[1/u,Reals]\}][[1]][[1]]] $\mathrm{p}->\left(\mathrm{a} \mathrm{E}^{\mathrm{ss}}(-1+)\right) /\left(\mathrm{E}^{2} \mathrm{ss}_{-}\right)$

Convert $Q(P)$ into $P(Q)$ using the parameters from the fit, and then discretize using the quantity adsorbed list. udd283=a (Ess $(-1+)) /\left(E^{2 ~ s s-}\right) /$.udnlm283["BestFitParameters"]/.n->plist; udd288=a ( $\left(E^{\text {ss }}(-1+)\right) /\left(E^{2 s s}\right.$ s-)/.udnlm288["BestFitParameters"]/.n->plist; udd293=a $\left(E^{\text {ss }}(-1+)\right) /\left(E^{2 s s}\right.$ s $) /$.udnlm293["BestFitParameters"]/n-n>plist; udd298=a $\left(E^{\text {ss }}(-1+)\right) /\left(E^{2 s s}\right.$ s $) /$.udnlm298["BestFitParameters"]/n-n->plist; udd308=a ( $\left(E^{\text {ss }}(-1+)\right) /\left(E^{2 s s}\right.$ - $) /$ udnlm308["BestFitParameters"]/n-n->plist; 


\section{The Clausius-Clapeyron equation uses $\log (P)$}

uddtot=Log[Transpose[\{udd283,udd288,udd293,udd298,udd308\}];; Load in the experimental temperatures. And invert for the Clausius-Clapeyron equation. temps $=\{283.15,288.15,293.15,298.15,308.15\}$; temps $=1 /$ temps;

Compute the errors from the nonlinear fits using the variance-covariance matrix and the Jacobian. jac $=\left\{\left\{\mathrm{D}\left[\mathrm{a}\left(\mathrm{E}^{\mathrm{ss}}(-1+)\right) /\left(\mathrm{E}^{2 \mathrm{ss}}\right)\right.\right.\right.$,u],D[a $\left.\left(\mathrm{E}^{\mathrm{ss}}(-1+)\right) /\left(\mathrm{E}^{2 \mathrm{ss}}-\right), \mathrm{ss}\right], \mathrm{D}\left[\mathrm{a}\left(\mathrm{E}^{\mathrm{ss}}(-1+)\right) /\left(\mathrm{E}^{2}\right.\right.$ ss-),a]\};;

e283=Simplify[jac.udnlm283["CovarianceMatrix"].Transpose[jac]/.udnlm283["Best FitParameters"]]/.n->plist;

e288=Simplify[jac.udnIm288["CovarianceMatrix"].Transpose[jac]/.udnIm288["Best FitParameters"]]/.n->plist;

e293=Simplify[jac.udnIm293["CovarianceMatrix"].Transpose[jac]/.udnlm293["Best FitParameters"]]/.n->plist;

e298=Simplify[jac.udnIm298["CovarianceMatrix"].Transpose[jac]/.udnlm298["Best FitParameters"]]/.n->plist;

e308=Simplify[jac.udnlm308["CovarianceMatrix"].Transpose[jac]/.udnlm308["Best FitParameters"]]/.n->plist;

Collect the errors into an array. errorsforcc $=\{\mathrm{e} 283[[1]], \mathrm{e} 288[[1]], \mathrm{e} 293[[1]], \mathrm{e} 298[[1]], \mathrm{e} 308[[1]]\}$;

Calculate the isosteric enthalpy, the error, and the residuals at each point.

$\mathrm{I}=\{\}$;

For[i $=1, \mathrm{i}<$ Length[plist] $+1, \mathrm{i}++$, AppendTo[l,Normal[NonlinearModelFit[Transpose@ $\{$ temps, uddtot[[i]]\},a+b* $x,\{a, b\}, x$, Weights-

$>1 /($ Transpose[Transpose[errorsforcc][[1]]][[i]])][[2]][[1]]]]

।

$\mathrm{e}=\{\}$;

For $[\mathrm{i}=1, \mathrm{i}<$ Length[plist] $+1, \mathrm{i}++$, AppendTo[e,NonlinearModelFit[Transpose@\{temps, uddtot[[i]]]\},a+b*a, $x, a, b\}, x$, Weights-

$>1 /($ Transpose[Transpose[errorsforcc $][[1]]][i]])][" P a r a m e t e r T a b l e "][[1]][1]][[3,3]]]]$

e

$r=\{\}$;

For $[\mathrm{i}=1, \mathrm{i}<$ Length[plist] $+1, \mathrm{i}++$, AppendTo[r,NonlinearModelFit[Transpose@\{temps, uddtot[[i]]\},a+b*a, $\{a, b\}, x$, Weights-

$>1 /($ Transpose[Transpose[errorsforcc][[1]]][ii]])]["RSquared"]]] 
$r$

$\{-4559.75,-4554.83,-4548.56,-4542.16,-4535.61,-4522.07,-4507.93,-4493.15,-$

$4477.68,-4461.45,-4444.4,-4426.53,-4407.86,-4388.4,-4368.1,-4346.82,-$

$4324.38,-4300.58,-4275.27,-4248.27,-4219.45,-4188.64,-4155.6,-4119.83,-$

$4080.33,-4035.66,-3985.42,-3931.9\}$

$\{25.3206,25.3358,25.3789,25.4496,25.5484,25.8281,26.2036,26.6358,27.0543,2$ $7.3607,27.4567,27.2938,26.9123,26.4364,26.0313,25.8579,26.044,26.671,27.77$ $44,29.3544,31.3886,33.8312,36.5797,39.3799,41.662,42.5048,41.3036,38.8782\}$

$\{0.999999,0.999997,0.999989,0.999962,0.999906,0.999971,0.999988,0.999993$, 0.999995,0.999996,0.999997,0.999998,0.999998,0.999998,0.999998,0.999999, 0.999999,0.999999,0.999999,0.999999,0.999999,0.999999,0.999999,0.999998, $0.999998,0.999999,0.999999,0.999999\}$

Plot the R2 from the Clausius-Clapeyron fit. ListPlot[Transpose@\{plist,r\},PlotTheme->"Scientific",FrameLabel->\{"Quantity Adsorbed (mmol/g)","Clausius-Clapeyron R $\left.{ }^{2 "}\right\}$,PlotLabel->"G = CO, X = Cl",ImageSize->Large,FrameStyle->Black]

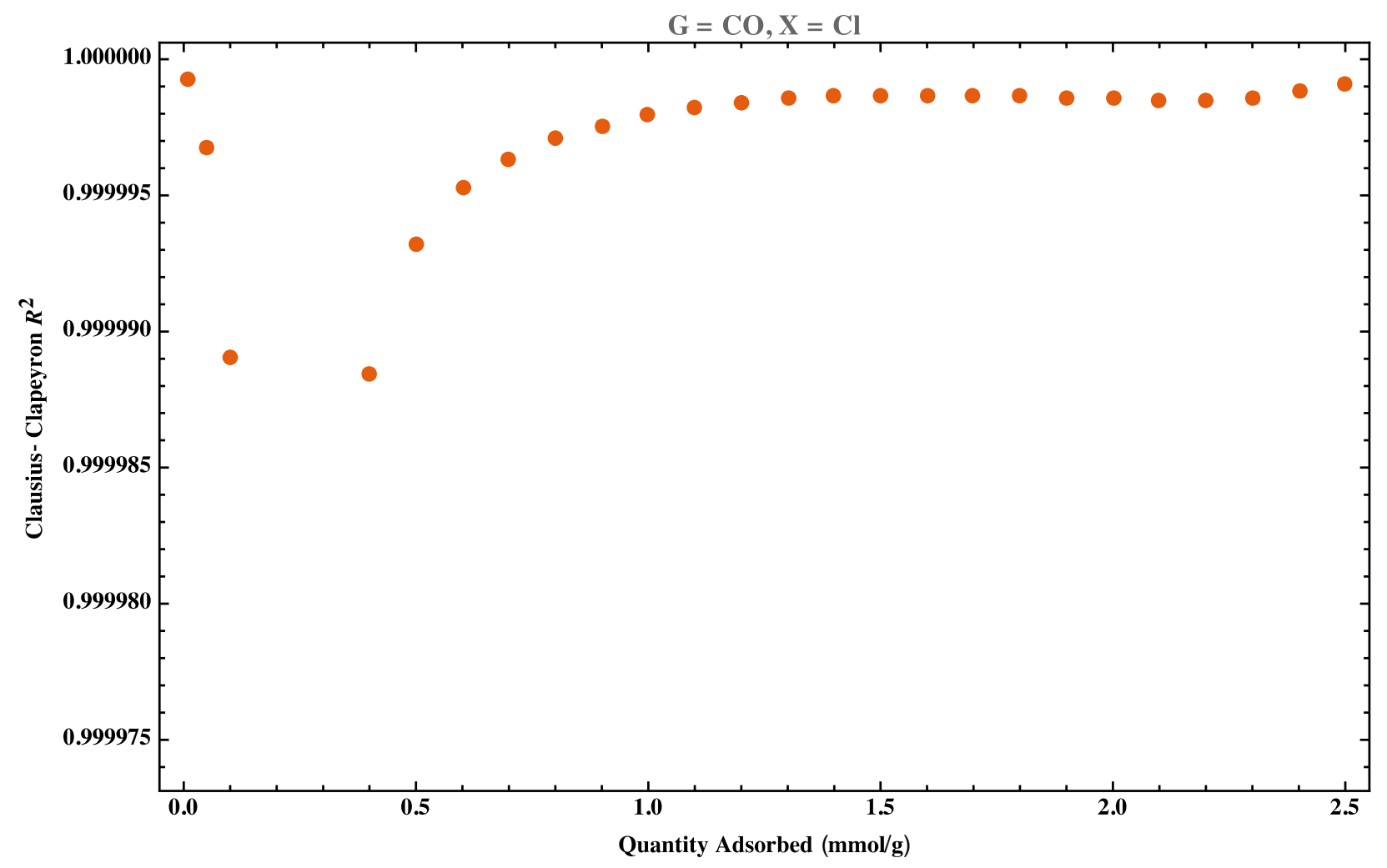

Convert results into units of $\mathrm{kJ} / \mathrm{mol}$

udds $\mathrm{h}=\mathrm{I}^{*}-8.31446261815324 / 1000$;

errudds $\mathrm{h}=\mathrm{e}^{*}-8.31446261815324 / 1000$; 
Plot all of the Clausius-Clapeyron fits, this requires the ErrorBarPlots module. Newer versions of Mathematica will have ErrorBarPlots deprecated and should use ListPlot instead.

Needs["ErrorBarPlots" "]

Show[Table[ListPlot[Transpose@\{temps,uddtot[[i]]\},PlotRange-

$>\{\{0.0032,0.0036\},\{-4,6\}\}$, PlotStyle->ColorData[i,"ColorList"],PlotTheme-

$>$ "Scientific",FrameLabel->\{"1/T (1/K)","log Pressure (kPa)"\},PlotLabel->"G = CO, $\mathrm{X}=$

Cl"],\{i, 1, Length[plist]\}],Table[Plot[Evaluate[Normal[NonlinearModelFitTranspose @ $\left\{\right.$ temps,uddtot[[i]]\},a+b $\mathrm{b}^{*},\{a, b\}, x$, Weights-

$>1 /$ (Transpose[Transpose[errorsforcc][[1]]][[i]])^2]]],\{x,0,1\},PlotStyle-

$>$ ColorData[i,"ColorList"]],\{i, 1,Length[plist]\}],Table[ErrorListPlot[Transpose@\{tem ps,uddtot[[i]],Transpose[Transpose[errorsforcc][[1]]][[i]]\},PlotStyle-

$>$ Directive[Opacity[0.5]], \{i, 1, Length[plist]\}],ImageSize->Large,FrameStyle$>$ Black]

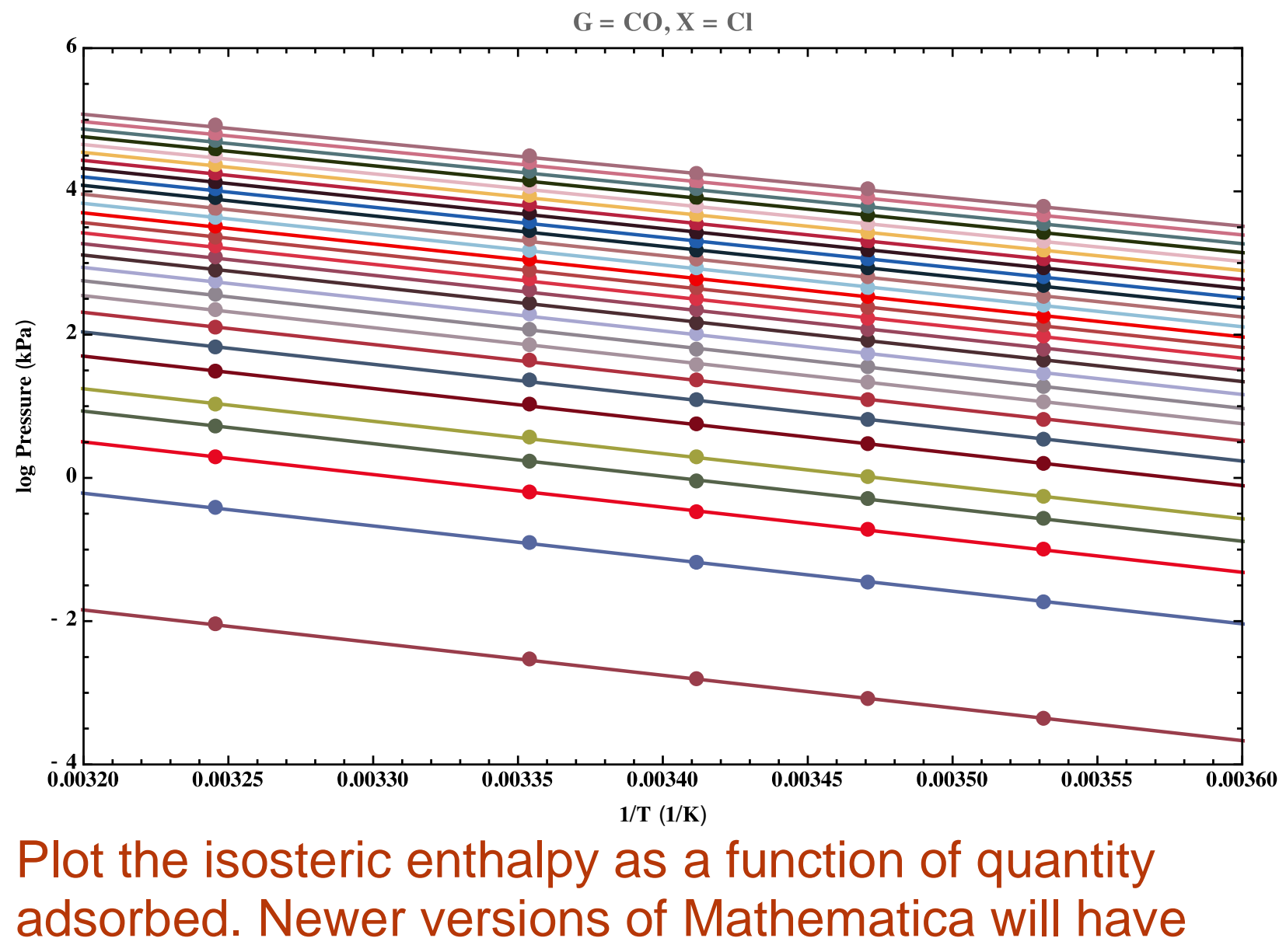


ErrorBarPlots deprecated and should use ListPlot instead.

Needs["ErrorBarPlots'"]

Show[ListPlot[Transpose@\{plist,uddslh\},PlotRange->\{\{0,2.55\},\{25,40\}\},PlotStyle$>$ Black,PlotTheme->"Scientific",FrameLabel->\{\{"- $\Delta \mathrm{H}(\mathrm{kJ} / \mathrm{mol}) "$, ,"'\},\{"Quantity Adsorbed (mmol/g)","G = CO, X = Cl"\}\},Frame->True,LabelStyle$>$ Directive[Black,FontSize$>$ 14]],ErrorListPlot[Transpose@\{plist,uddslh,Abs[erruddsIh]\},PlotStyle>Directive[Opacity[0.5],Red]],ImageSize->Large,FrameStyle->Black]

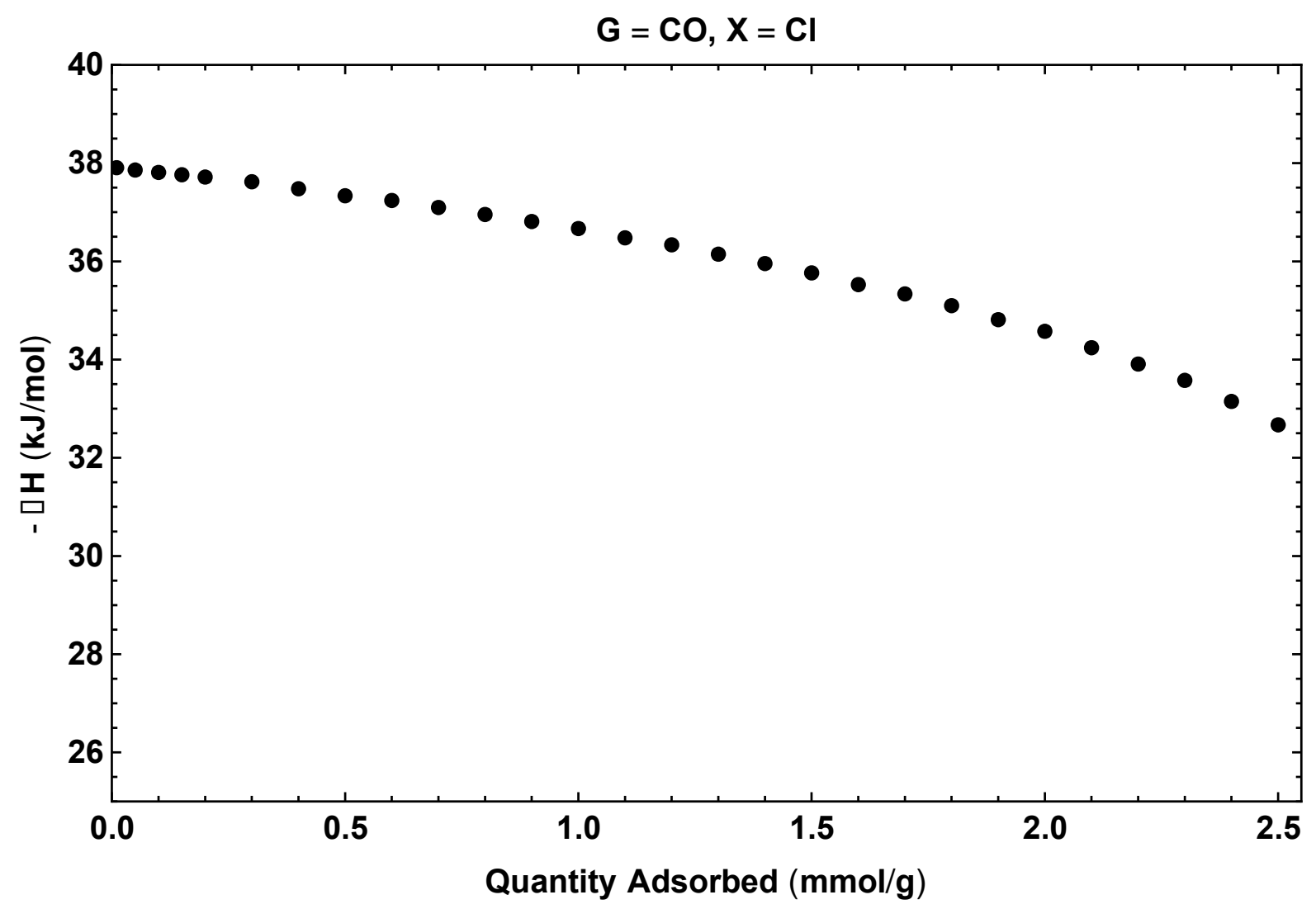

Store results into uniquely named variables. This is useful if working across multiple notebook pages. coclplist=plist cocl=uddslh errcocl=erruddslh

$\{0.01,0.05,0.1,0.15,0.2,0.3,0.4,0.5,0.6,0.7,0.8,0.9,1 ., 1.1,1.2,1.3,1.4,1.5,1.6,1.7,1$. $8,1.9,2 ., 2.1,2 \cdot 2,2 \cdot 3,2.4,2.5\}$ 
$\{37.9119,37.871,37.8189,37.7656,37.7111,37.5986,37.481,37.3582,37.2295,37$. $0945,36.9528,36.8042,36.649,36.4872,36.3184,36.1415,35.9549,35.757,35.546$ $5,35.3221,35.0825,34.8263,34.5516,34.2541,33.9258,33.5544,33.1366,32.6917\}$ $\{-0.210527,-0.210654,-0.211012,-0.2116,-0.212421,-0.214747,-0.217869,-$ $0.221463,-0.224942,-0.227489,-0.228288,-0.226934,-0.223761,-0.219804,-$ $0.216436,-0.214994,-0.216542,-0.221755,-0.23093,-0.244066,-0.260979,-$ $0.281288,-0.304141,-0.327422,-0.346397,-0.353405,-0.343418,-0.323251\}$ 


\section{Section S7. References.}

(1) Rieth, A. J.; Wright, A. M.; Skorupskii, G.; Mancuso, J. L.; Hendon, C. H.; Dincă, M. Record-Setting Sorbents for Reversible Water Uptake by Systematic Anion Exchanges in Metal-Organic Frameworks. Journal of the American Chemical Society 2019, 141 (35), 13858-13866.

(2) Kresse, G.; Furthmüller, J. Efficient Iterative Schemes for Ab Initio Total-Energy Calculations Using a Plane-Wave Basis Set. Physical review B 1996, 54 (16), 11169.

(3) Perdew, J. P.; Ruzsinszky, A.; Csonka, G. I.; Vydrov, O. A.; Scuseria, G. E.; Constantin, L. A.; Zhou, X.; Burke, K. Restoring the Density-Gradient Expansion for Exchange in Solids and Surfaces. Physical review letters 2008, 100 (13), 136406.

(4) Zecchina, A.; Scarano, D.; Bordiga, S.; Spoto, G.; Lamberti, C. Surface Structures of Oxides and Halides and Their Relationships to Catalytic Properties. 2001.

(5) Bloch, E. D.; Hudson, M. R.; Mason, J. A.; Chavan, S.; Crocellà, V.; Howe, J. D.; Lee, K.; Dzubak, A. L.; Queen, W. L.; Zadrozny, J. M.; others. Reversible CO Binding Enables Tunable $\mathrm{CO} / \mathrm{H} 2$ and $\mathrm{CO} / \mathrm{N} 2$ Separations in Metal-Organic Frameworks with Exposed Divalent Metal Cations. Journal of the American Chemical Society 2014, 136 (30), 10752-10761.

(6) Miller, S. R.; Pearce, G. M.; Wright, P. A.; Bonino, F.; Chavan, S.; Bordiga, S.; Margiolaki, I.; Guillou, N.; Férey, G.; Bourrelly, S.; others. Structural Transformations and Adsorption of Fuel-Related Gases of a Structurally Responsive Nickel Phosphonate Metal- Organic Framework, Ni-STA-12. Journal of the American Chemical Society 2008, 130 (47), 15967-15981.

(7) Saha, D.; Deng, S. Adsorption Equilibria and Kinetics of Carbon Monoxide on Zeolite 5A, 13X, MOF-5, and MOF-177. Journal of Chemical \& Engineering Data 2009, 54 (8), 2245-2250.

(8) Peng, J.; Xian, S.; Xiao, J.; Huang, Y.; Xia, Q.; Wang, H.; Li, Z. A Supported Cu (I)@ MIL-100 (Fe) Adsorbent with High CO Adsorption Capacity and CO/N2 Selectivity. Chemical Engineering Journal 2015, 270, 282-289.

(9) Deng, S.; Lin, Y. Sol-Gel Preparation and Properties of Alumina Adsorbents for Gas Separation. AlChE Journal 1995, 41 (3), 559-570.

(10) Valenzano, L.; Civalleri, B.; Chavan, S.; Palomino, G. T.; Areán, C. O.; Bordiga, S. Computational and Experimental Studies on the Adsorption of $\mathrm{CO}, \mathrm{N} 2$, and $\mathrm{CO} 2$ on Mg-MOF-74. The Journal of Physical Chemistry C 2010, 114 (25), 1118511191.

(11) Chang, G.; Huang, M.; Su, Y.; Xing, H.; Su, B.; Zhang, Z.; Yang, Q.; Yang, Y.; Ren, Q.; Bao, Z.; others. Immobilization of Ag (i) into a Metal-Organic Framework withSO $3 \mathrm{H}$ Sites for Highly Selective Olefin-Paraffin Separation at Room Temperature. Chemical Communications 2015, 51 (14), 2859-2862.

(12) Li, B.; Zhang, Y.; Ma, D.; Wu, Z.; Ma, S. Ag (I) Ion Functionalized Porous Organic Polymers As a New Platform for Highly Selective Adsorption of Ethylene over Ethane. Journal of the American Chemical Society 2014, 136 (24).

(13) Zhang, Y.; Li, B.; Krishna, R.; Wu, Z.; Ma, D.; Shi, Z.; Pham, T.; Forrest, K.; Space, B.; Ma, S. Highly Selective Adsorption of Ethylene over Ethane in a MOF Featuring 


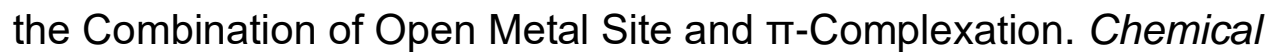
communications 2015, 51 (13), 2714-2717.

(14) Wang, Y.; Hu, Z.; Cheng, Y.; Zhao, D. Silver-Decorated Hafnium Metal-Organic Framework for Ethylene/Ethane Separation. Industrial \& Engineering Chemistry Research 2017, 56 (15), 4508-4516.

(15) Liao, Y.; Zhang, L.; Weston, M. H.; Morris, W.; Hupp, J. T.; Farha, O. K. Tuning Ethylene Gas Adsorption via Metal Node Modulation: Cu-MOF-74 for a High Ethylene Deliverable Capacity. Chemical Communications 2017, 53 (67), 93769379.

(16) Bloch, E. D.; Queen, W. L.; Krishna, R.; Zadrozny, J. M.; Brown, C. M.; Long, J. R. Hydrocarbon Separations in a Metal-Organic Framework with Open Iron (II) Coordination Sites. science 2012, 335 (6076), 1606-1610.

(17) Bao, Z.; Alnemrat, S.; Yu, L.; Vasiliev, I.; Ren, Q.; Lu, X.; Deng, S. Adsorption of Ethane, Ethylene, Propane, and Propylene on a Magnesium-Based Metal-Organic Framework. Langmuir 2011, 27 (22), 13554-13562.

(18) Chang, G.; Bao, Z.; Ren, Q.; Deng, S.; Zhang, Z.; Su, B.; Xing, H.; Yang, Y. Fabrication of Cuprous Nanoparticles in MIL-101: An Efficient Adsorbent for the Separation of Olefin-Paraffin Mixtures. RSC advances 2014, 4 (39), 20230-20233.

(19) Chen, Y.; Wu, H.; Lv, D.; Shi, R.; Chen, Y.; Xia, Q.; Li, Z. Highly Adsorptive Separation of Ethane/Ethylene by an Ethane-Selective MOF MIL-142A. Industrial \& Engineering Chemistry Research 2018, 57 (11), 4063-4069.

(20) Chen, Y.; Qiao, Z.; Wu, H.; Lv, D.; Shi, R.; Xia, Q.; Zhou, J.; Li, Z. An EthaneTrapping MOF PCN-250 for Highly Selective Adsorption of Ethane over Ethylene. Chemical Engineering Science 2018, 175, 110-117.

(21) Skopp, J. Derivation of the Freundlich Adsorption Isotherm from Kinetics. Journal of Chemical Education 2009, 86 (11), 1341.

(22) Sreńscek-Nazzal, J.; Narkiewicz, U.; Morawski, A. W.; Wróbel, R. J.; Michalkiewicz, B. Comparison of Optimized Isotherm Models and Error Functions for Carbon Dioxide Adsorption on Activated Carbon. Journal of Chemical \& Engineering Data 2015, 60 (11), 3148-3158.

(23) Ochoa, B.; Belongie, S. Covariance Propagation for Guided Matching. In Proceedings of the Workshop on Statistical Methods in Multi-Image and Video Processing (SMVP); 2006; Vol. 83. 